\title{
Anomalous Localized Resonance Phenomena in the Nonmagnetic, Finite-Frequency Regime
}

\author{
Daniel Onofrei ${ }^{1}$ and Andrew E. Thaler ${ }^{2}$ \\ ${ }^{1}$ Department of Mathematics, University of Houston, Houston, TX 77204, USA \\ ${ }^{2}$ Institute for Mathematics and Its Applications, University of Minnesota, College of Science and Engineering, Minneapolis, \\ MN 55455, USA
}

Correspondence should be addressed to Daniel Onofrei; onofrei@math.uh.edu

Received 23 May 2016; Accepted 7 August 2016

Academic Editor: Jacopo Bellazzini

Copyright (c) 2016 D. Onofrei and A. E. Thaler. This is an open access article distributed under the Creative Commons Attribution License, which permits unrestricted use, distribution, and reproduction in any medium, provided the original work is properly cited.

\begin{abstract}
The phenomenon of anomalous localized resonance (ALR) is observed at the interface between materials with positive and negative material parameters and is characterized by the fact that when a given source is placed near the interface, the electric and magnetic fields start to have very fast and large oscillations around the interface as the absorption in the materials becomes very small while they remain smooth and regular away from the interface. In this paper, we discuss the phenomenon of anomalous localized resonance (ALR) in the context of an infinite slab of homogeneous, nonmagnetic material $(\mu=1)$ with permittivity $\epsilon_{s}=-1-\mathrm{i} \delta$ for some small loss $\delta \ll 1$ surrounded by positive, nonmagnetic, homogeneous media. We explicitly characterize the limit value of the product between frequency and the width of slab beyond which the ALR phenomenon does not occur and analyze the situation when the phenomenon is observed. In addition, we also construct sources for which the ALR phenomenon never appears.
\end{abstract}

\section{Introduction}

In the following, we discuss the anomalous localized resonance phenomenon (ALR) appearing at the interface between materials with positive and negative material parameters in the finite-frequency regime. We consider the particular slab geometry described by (see Figure 1)

$$
\begin{aligned}
& \mathscr{C} \equiv\left\{(x, y) \in \mathbb{R}^{2}: x<0\right\} ; \\
& \mathcal{S} \equiv\left\{(x, y) \in \mathbb{R}^{2}: 0<x<a\right\} ; \\
& \mathscr{M} \equiv\left\{(x, y) \in \mathbb{R}^{2}: x>a\right\},
\end{aligned}
$$

where $a>0$ denotes the width of the slab and the sets $\mathscr{C}, \mathcal{S}$, and $\mathscr{M}$ represent the regions to the left of the slab, within the slab, and to the right of the slab, respectively. We also define

$$
\begin{aligned}
& d_{0} \equiv \min \{x:(x, y) \in \operatorname{supp} f\}, \\
& d_{1} \equiv \max \{x:(x, y) \in \operatorname{supp} f\} .
\end{aligned}
$$

In this geometry, we assume that all materials are homogeneous and nonmagnetic (i.e., with magnetic permeability $\mu=1$ ); the electrical permittivity is given by

$$
\varepsilon \equiv \begin{cases}1 & \text { for } x<0 \\ -1-\mathrm{i} \delta & \text { for } 0<x<a \\ 1 & \text { for } x>a\end{cases}
$$

for some $\delta \in(0,1)$. We consider the following partial differential equation (PDE) in $2 \mathrm{D}$ :

$$
\nabla \cdot\left(\frac{1}{\varepsilon} \nabla V\right)+\left(k_{0}^{2}+\mathrm{i} \zeta\right) V=-f \quad \text { in } \mathbb{R}^{2}
$$

where $\zeta \geq 0, k_{0}>0, f \in L^{2}(\mathscr{M})$ with compact support in $\mathscr{M}$, and $\varepsilon$ is given in (3) (see Section A.5 for a derivation of (4) from the Maxwell equations). 


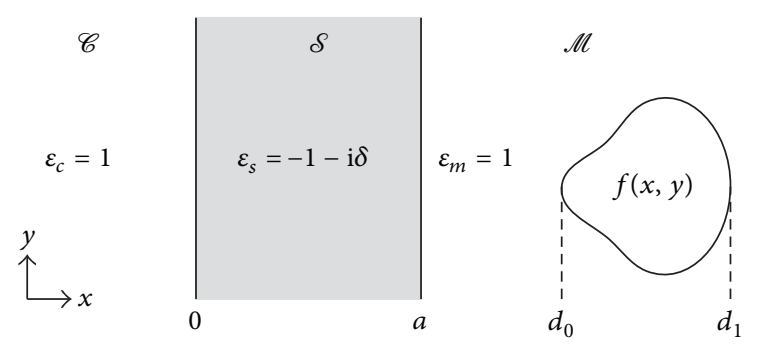

FIGURE 1: In this figure, we illustrate the geometry of the problem we consider in this paper.

For convenience, we define

$$
\begin{aligned}
V_{c} & \left.\equiv V\right|_{\mathscr{C}} ; \\
V_{s} & \left.\equiv V\right|_{\mathcal{S}} ; \\
V_{m} & \left.\equiv V\right|_{\mathscr{M}} .
\end{aligned}
$$

We assume the solution $V$ also satisfies the following continuity conditions across the boundaries at $x=0$ and $x=a$ for almost every $y \in \mathbb{R}$ :

$$
\begin{aligned}
V_{c}(0, y) & =V_{s}(0, y) \\
\frac{\partial V_{c}}{\partial x}(0, y) & =\frac{1}{-1-\mathrm{i} \delta} \frac{\partial V_{s}}{\partial x}(0, y), \\
V_{s}(a, y) & =V_{m}(a, y) \\
\frac{1}{-1-\mathrm{i} \delta} \frac{\partial V_{s}}{\partial x}(a, y) & =\frac{\partial V_{m}}{\partial x}(a, y) .
\end{aligned}
$$

In what follows we assume that the parameters and data are such that problem (4) and (6) admit a unique solution $V \in$ $L_{\text {loc }}^{2}\left(\mathbb{R}^{2}\right)$ with $V(x, \cdot) \in H^{1}(\mathbb{R})$ and $(\partial V / \partial x)(x, \cdot) \in L^{2}(\mathbb{R})$ for almost every $x \in \mathbb{R}$.

Remark 1. Note that, in the case when $\zeta>0$, the unique solution of the problem will have the property that $V(x, y) \rightarrow$ 0 as $|x| \rightarrow \infty$ for almost every $y \in \mathbb{R}$; for $\zeta \ll 1$, this solution will be well approximated by the solution in the case $\zeta=0$.

We say anomalous localized resonance (ALR) occurs if the following two properties hold as $\delta \rightarrow 0^{+}[1]$ :

(1) $|V| \rightarrow \infty$ in certain localized regions with boundaries that are not defined by discontinuities in the relative permittivity.

(2) $V$ approaches a smooth limit outside these localized regions.

In [1], Milton et al. showed that if $f$ is a dipole and $\varepsilon_{c}=\varepsilon_{m}=1$, then ALR occurs if $a<d_{0}<2 a$, where $d_{0}$ is the location of the dipole. In this case, there are two locally resonant stripsone centered on each face of the slab. As the loss parameter (represented by $\delta$ ) tends to zero, the potential diverges and oscillates wildly in these resonant regions. Outside these regions, the potential converges to a smooth function. Also, if the source is far enough away from the slab, that is, if $d_{0}>2 a$, then there is no resonance and again the potential converges to a smooth function.

Applications of ALR to superlensing were first discussed by Nicorovici et al. in [2] and were analyzed in more depth in [1] (see also the works by Yan et al. [3], Bergman [4], Nguyen [5], Pendry [6], and Pendry and Ramakrishna [7] for a description of superlensing phenomena).

Applications of ALR to cloaking in the quasistatic regime were first analyzed Milton and Nicorovici [8]; they showed that if $\varepsilon_{c}=\varepsilon_{m}=1$ and a fixed field is applied to the system (e.g., a uniform field at infinity), then a polarizable dipole located in the region $a<d_{0}<3 a / 2$ causes anomalous localized resonance and is cloaked in the limit $\delta \rightarrow 0^{+}$. Cloaking due to anomalous localized resonance (CALR) in the quasistatic regime was further discussed in [9-17]. CALR in the long-time limit regime was discussed in $[16,18]$ (see also [19]).

In [20], Nicorovici et al. studied CALR for the circular cylindrical superlens in the finite-frequency case; they showed that for small values of $\delta$ the cloaking device (the superlens) can effectively cloak a tiny cylindrical inclusion located within the cloaking region but that the superlens does not necessarily cloak itself-they deemed this phenomenon to be the "ostrich effect." The finite-frequency case was further discussed by Kettunen et al. [21] and Nguyen [22].

In the present report we prove, analytically and numerically, the existence of a limit value $\gamma_{*}$, such that, for $k_{0}$ with $k_{0} a>\gamma_{*}$, ALR does not occur regardless of the position of the source with respect to the slab interface. Under suitable conditions on the source, we present numerical evidence for the occurrence of ALR in the regime $k_{0} a<\gamma_{*}$ when the source is close enough to the material interface, and we discuss some characteristics of the phenomenon in this frequency regime as well. In the end we present two examples of sources $f$ which do not generate ALR regardless of the frequency regime and their relative position with respect to the material interface.

The paper is organized as follows: in Section 1.1 we present highlights of the derivation of the unique solution in the Fourier domain while in Section 1.2 we describe the energy around the right interface of the slab. In Section 3, we show the absence of ALR phenomena for large enough values of $k_{0} a$ while in Section 3.2 we present an interesting side effect of the nonmagnetic case, namely, the shielding effect of the slab which behaves as an almost perfect reflector. Next, for suitable conditions on the source, in Section 4.1 we present numerical evidence for the ALR phenomenon in the case of small enough values of $k_{0} a$. In Section 4.2 , we construct two examples of possible sources for which there is no ALR phenomenon regardless of the range of $k_{0} a$ or the relative position of the source with respect to the slab interface. The Appendix contains the technical proofs and derivations which were not included in the main text.

1.1. Solution in Fourier Domain. Due to our well-posedness assumption, it follows that our problem will admit a unique solution after applying the Fourier transform with respect to the $y$ variable. Recall that, for a given function $h(x, \cdot) \in L^{2}(\mathbb{R})$ 
for some $x \in \mathbb{R}$, the Fourier transform of $h$ with respect to $y$ is

$$
\widehat{h}(x, q)=\int_{-\infty}^{\infty} h(x, y) \mathrm{e}^{-\mathrm{i} q y} \mathrm{~d} y .
$$

We will study the Fourier domain solution in each of the relevant subdomains defined in (1).

1.1.1. The Solution in $\mathscr{C}$. In the region $\mathscr{C}$, the relevant equation is

$$
\frac{\partial^{2} V_{c}}{\partial x^{2}}+\frac{\partial^{2} V_{c}}{\partial y^{2}}+k_{0}^{2} V_{c}=0
$$

Taking the Fourier transform of (8) with respect to $y$, we find that $\widehat{V}_{c}(x, q)$ satisfies

$$
\frac{\partial^{2} \widehat{V}_{c}}{\partial x^{2}}-k_{0}^{2} v_{c}^{2} \widehat{V}_{c}=0, \quad \text { where } v_{c}^{2} \equiv \frac{q^{2}}{k_{0}^{2}}-1
$$

Remark 2. Here and throughout the paper, we take the principal square root of complex numbers; that is, for a complex number $z=z^{\prime}+\mathrm{i} z^{\prime \prime}=|z| \mathrm{e}^{\mathrm{i} \theta}$, where $\theta \in(-\pi, \pi]$, we take

$$
\sqrt{z}=|z|^{1 / 2} \mathrm{e}^{\mathrm{i} \theta / 2}
$$

where $\theta / 2 \in(-\pi / 2, \pi / 2]$. In particular, this implies $\operatorname{Re} \sqrt{z} \geq$ 0 .

\section{Remark 2 implies}

$$
\nu_{c}= \begin{cases}\mathrm{i} \sqrt{\left|\frac{q^{2}}{k_{0}^{2}}-1\right|} & \text { if } \frac{q^{2}}{k_{0}^{2}}<1, \\ \sqrt{\frac{q^{2}}{k_{0}^{2}}-1} & \text { if } \frac{q^{2}}{k_{0}^{2}} \geq 1 .\end{cases}
$$

Then the general solution to (9) is

$$
\widehat{V}_{c}(x, q)=A_{q} \mathrm{e}^{k_{0} v_{c} x}+B_{q} \mathrm{e}^{-k_{0} v_{c} x}
$$

for coefficients $A_{q}$ and $B_{q}$ that are independent of $x$.

If $q^{2} / k_{0}^{2}<1$, then $\nu_{c}$ is purely imaginary. Because $V_{c}$ should be outgoing (i.e., left-going) as $x \rightarrow-\infty$ and we are considering $\mathrm{e}^{\mathrm{i} \omega t}$ time dependence (see Section A.5), we should have

$$
\widehat{V}_{c} \sim \mathrm{e}^{\mathrm{i} k_{0} \sqrt{\left|q^{2} / k_{0}^{2}-1\right|} x} \quad \text { as } x \longrightarrow-\infty
$$

From (11) and (12), we see that we can ensure this by taking $B_{q}=0$.

On the other hand, if $q^{2} / k_{0}^{2}>1$, then $\nu_{c}>0$. Thus we take $B_{q}=0$ in this case to ensure that $\widehat{V}_{c}(x, q) \rightarrow 0$ as $x \rightarrow \infty$. Finally, without loss of generality we may also take $B_{q}=0$ for $q^{2} / k_{0}^{2}=1$. Therefore,

$$
\widehat{V}_{c}(x, q)=A_{q} \mathrm{e}^{k_{0} v_{c} x} .
$$

1.1.2. The Solution in $\mathcal{S}$. In the region $\mathcal{S}$, the Fourier transform of $V_{\mathrm{s}}$ satisfies

$$
\frac{\partial^{2} \widehat{V}_{s}}{\partial x^{2}}-k_{0}^{2} v_{s}^{2} \widehat{V}_{s}=0, \quad \text { where } v_{s}^{2} \equiv\left(\frac{q^{2}}{k_{0}^{2}}+1\right)+\mathrm{i} \delta .
$$

The general solution is

$$
\widehat{V}_{s}(x, q)=C_{q} \mathrm{e}^{k_{0} v_{s} x}+D_{q} \mathrm{e}^{-k_{0} v_{s} x} ;
$$

the coefficients $C_{q}$ and $D_{q}$ may be found by using the continuity conditions across $x=0$ from (6). In particular, we find

$$
\widehat{V}_{s}(x, q)=A_{q}\left(\frac{\alpha+1}{2 \alpha}\right) \mathrm{e}^{k_{0} v_{s} x}\left(1+\operatorname{Re}^{-2 k_{0} v_{s} x}\right),
$$

where

$$
\begin{aligned}
& \alpha \equiv \frac{\nu_{s}}{(-1-\mathrm{i} \delta) \nu_{c}}, \\
& R \equiv \frac{\alpha-1}{\alpha+1}=\frac{\nu_{s}+(1+\mathrm{i} \delta) \nu_{c}}{\nu_{s}-(1+\mathrm{i} \delta) \nu_{c}} .
\end{aligned}
$$

Although one can observe that $\alpha$ degenerates for $q^{2}=k_{0}^{2}$ we will see in (25) and (27) that $A_{q} / \alpha$ is well defined in the limit when $q^{2}=k_{0}^{2}$.

1.1.3. The Solution in $\mathscr{M}$. In the region $\mathscr{M}$, the Fourier transform of $V_{m}$ satisfies

$$
\frac{\partial^{2} \widehat{V}_{m}}{\partial x^{2}}-k_{0}^{2} v_{m}^{2} \widehat{V}_{m}=-\widehat{f}(x, q), \quad \text { where } v_{m}^{2} \equiv \frac{q^{2}}{k_{0}^{2}}-1 \text {. }
$$

If $q^{2} / k_{0}^{2} \neq 1$, then the general solution to (19) can be found using the Laplace transform and the continuity conditions across $x=a$ from $(6)[23,24]$; we have

$$
\begin{gathered}
\widehat{V}_{m}(x, q)=\frac{\mathrm{e}^{k_{0} v_{m} x}}{2}\left[A_{q} \mathrm{e}^{-k_{0} v_{m} a}\left(\psi_{q}^{+}+\frac{\psi_{q}^{-}}{v_{m}}\right)\right. \\
\left.-\frac{1}{k_{0} v_{m}} \int_{d_{0}}^{x} \mathrm{e}^{-k_{0} v_{m} s} \widehat{f}(s, q) \mathrm{d} s\right] \\
+\frac{\mathrm{e}^{-k_{0} v_{m} x}}{2}\left[A_{q} \mathrm{e}^{k_{0} v_{m} a}\left(\psi_{q}^{+}+\frac{\psi_{q}^{-}}{v_{m}}\right)\right. \\
\left.+\frac{1}{k_{0} v_{m}} \int_{d_{0}}^{x} \mathrm{e}^{k_{0} v_{m} s} \hat{f}(s, q) \mathrm{d} s\right],
\end{gathered}
$$

where

$$
\begin{aligned}
\psi_{q}^{+} & \equiv \frac{1}{A_{q}} \widehat{V}_{s}(a, q)=\left(\frac{\alpha+1}{2 \alpha}\right) \mathrm{e}^{k_{0} v_{s} a}\left(1+R \mathrm{e}^{-2 k_{0} v_{s} a}\right) ; \\
\psi_{q}^{-} & \equiv \frac{1}{k_{0} A_{q}}\left(\frac{1}{(-1-\mathrm{i} \delta)} \frac{\partial \widehat{V}_{s}}{\partial x}(a, q)\right) \\
& =\left(\frac{v_{s}}{-1-\mathrm{i} \delta}\right)\left(\frac{\alpha+1}{2 \alpha}\right) \mathrm{e}^{k_{0} v_{s} a}\left(1-\operatorname{Re}^{-2 k_{0} v_{s} a}\right) .
\end{aligned}
$$


If $q^{2} / k_{0}^{2}<1$, then $v_{m}$ is purely imaginary. Because $V_{m}$ should be outgoing (i.e., rightgoing) as $x \rightarrow \infty$ and we are considering $\mathrm{e}^{\mathrm{i} \omega t}$ time dependence, we should have

$$
\widehat{V}_{m} \sim \mathrm{e}^{-\mathrm{i} k_{0} \sqrt{\left|q^{2} / k_{0}^{2}-1\right|} x} \text { as } x \longrightarrow \infty .
$$

To ensure this, we take the first expression in brackets in (20) to be zero and find that

$$
A_{q} \equiv \frac{I_{q} e^{k_{0} v_{m} a}}{k_{0}\left(v_{m} \psi_{q}^{+}+\psi_{q}^{-}\right)},
$$

where

$$
I_{q} \equiv \int_{d_{0}}^{d_{1}} \widehat{f}(s, q) \mathrm{e}^{-k_{0} v_{m} s} \mathrm{~d} s
$$

If $q^{2} / k_{0}^{2}>1$, then $v_{m}>0$; to ensure that $\widehat{V}_{m}(x, q) \rightarrow 0$ as $x \rightarrow \infty$, we again take $A_{q}$ as in (23).

Finally, if $q^{2} / k_{0}^{2}=1$, then we can use the Laplace transform and the continuity conditions across $x=a$ to find that

$$
\begin{aligned}
\widehat{V}_{m}\left(x, \pm k_{0}\right)= & A_{ \pm k_{0}}\left(\psi_{ \pm k_{0}}^{+}-a \psi_{ \pm k_{0}}^{-}\right) \\
& +\int_{d_{0}}^{x} s \widehat{f}\left(s, \pm k_{0}\right) \mathrm{d} s \\
& +x\left[k_{0} A_{ \pm k_{0}} \psi_{ \pm k_{0}}^{-}-\int_{d_{0}}^{x} \hat{f}\left(s, \pm k_{0}\right) \mathrm{d} s\right],
\end{aligned}
$$

where

$$
\begin{aligned}
& \psi_{ \pm k_{0}}^{+}=\left(1+\mathrm{e}^{-2 k_{0} v_{s} a}\right) ; \\
& \psi_{ \pm k_{0}}^{-}=\left(\frac{v_{s}}{-1-\mathrm{i} \delta}\right)\left(1-\mathrm{e}^{-2 k_{0} v_{s} a}\right)
\end{aligned}
$$

with $v_{s}$ defined at (15) being computed for $q= \pm k_{0}$ and where again we take $A_{ \pm k_{0}}$ so that we ensure $\widehat{V}_{m}$ is outgoing as $x \rightarrow$ $\infty$; in this case

$$
A_{ \pm k_{0}}=\frac{1}{k_{0} \psi_{ \pm k_{0}}^{-}} \int_{d_{0}}^{d_{1}} \hat{f}\left(s, \pm k_{0}\right) \mathrm{d} s .
$$

1.2. Energy Discussion. For $0<\xi \leq a$, we define the strip

$$
S_{\xi} \equiv\left\{(x, y) \in \mathbb{R}^{2}: a-\xi<x<a\right\} .
$$

Then, due to the Plancherel theorem and properties of Fourier transforms, we have

$$
\begin{aligned}
\|\nabla V\|_{L^{2}\left(S_{\xi}\right)}^{2} & =\int_{a-\xi}^{a} \int_{-\infty}^{\infty}\left|\nabla V_{s}(x, y)\right|^{2} \mathrm{~d} y \mathrm{~d} x \\
& =\int_{a-\xi}^{a} \int_{-\infty}^{\infty}\left|\frac{\partial^{2} V_{s}}{\partial x^{2}}\right|^{2}+\left|\frac{\partial^{2} V_{s}}{\partial y^{2}}\right|^{2} \mathrm{~d} y \mathrm{~d} x \\
& =\frac{1}{2 \pi} \int_{a-\xi}^{a} \int_{-\infty}^{\infty}\left|\frac{\partial^{2} \widehat{V}_{s}}{\partial x^{2}}\right|^{2}+|q|^{2}\left|\widehat{V}_{s}\right|^{2} \mathrm{~d} q \mathrm{~d} x .
\end{aligned}
$$

Using (17)-(18) and (21)-(24) in this expression, switching the order of integration, computing the integral with respect to $x$, using the fact that $\left|\nabla V_{s}\right|^{2}$ is an even function of $q$ if $f$ is real-valued, making the change of variables $p=q / k_{0}$, and simplifying the resulting expression, we obtain

$$
\begin{aligned}
& E_{\delta}(\xi) \equiv\|\nabla V\|_{L^{2}\left(S_{\xi}\right)}^{2}=\frac{1+\delta^{2}}{\pi} \\
& \cdot \int_{0}^{\infty} \frac{\left|I_{p}\right|^{2} \mathrm{e}^{2 k_{0} v_{m}^{\prime} a}\left|\nu_{s}-(1+\mathrm{i} \delta) v_{m}\right|^{2}}{\left|g_{\delta}(p ; \gamma)\right|^{2}} \cdot\left\{\left(\left|\nu_{s}\right|^{2}\right.\right. \\
& \left.\quad+|p|^{2}\right)\left[\left(\frac{1-\mathrm{e}^{-2 k_{0} v_{s}^{\prime} \xi}}{2 v_{s}^{\prime}}\right)\right. \\
& \left.+|R|^{2} \mathrm{e}^{-4 k_{0} v_{s}^{\prime}(a-\xi / 2)}\left(\frac{1-\mathrm{e}^{-2 k_{0} v_{s}^{\prime} \xi}}{2 v_{s}^{\prime}}\right)\right]+2\left(-\left|\nu_{s}\right|^{2}\right. \\
& \left.+|p|^{2}\right) \mathrm{e}^{-2 k_{0} v_{s}^{\prime} a} \\
& \left.\cdot \operatorname{Im}\left[\bar{R} \mathrm{e}^{2 \mathrm{i} k_{0} v_{s}^{\prime \prime} a}\left(\frac{1-\mathrm{e}^{-2 \mathrm{i} k_{0} v_{s}^{\prime \prime} \xi}}{2 v_{s}^{\prime \prime}}\right)\right]\right\} \mathrm{d} p,
\end{aligned}
$$

where

$$
\begin{aligned}
g_{\delta}(p ; \gamma) \equiv & {\left[v_{s}-(1+\mathrm{i} \delta) v_{m}\right]^{2} } \\
& -\left[v_{s}+(1+\mathrm{i} \delta) v_{m}\right]^{2} \mathrm{e}^{-2 \gamma v_{s}},
\end{aligned}
$$

we have used the fact that $v_{c}=v_{m}$ (see (11) and (19)), and we have replaced $q$ by $k_{0} p$ throughout the integrand (e.g., we have $\left.v_{m}=\sqrt{p^{2}-1}\right)$.

Similarly, we have

$$
\begin{aligned}
& \|V\|_{L^{2}\left(S_{\xi}\right)}^{2}=\frac{1+\delta^{2}}{\pi} \int_{0}^{\infty} \frac{\left|I_{p}\right|^{2} \mathrm{e}^{2 k_{0} \nu_{m}^{\prime} a}\left|\nu_{s}-(1+\mathrm{i} \delta) v_{m}\right|^{2}}{\left|g_{\delta}(p ; \gamma)\right|^{2}} \\
& \cdot\left\{\left[\left(\frac{1-\mathrm{e}^{-2 k_{0} v_{s}^{\prime} \xi}}{2 v_{s}^{\prime}}\right)\right.\right. \\
& \left.+|R|^{2} \mathrm{e}^{-4 k_{0} v_{s}^{\prime}(a-\xi / 2)}\left(\frac{1-\mathrm{e}^{-2 k_{0} v_{s}^{\prime} \xi}}{2 v_{s}^{\prime}}\right)\right]+2 \mathrm{e}^{-2 k_{0} v_{s}^{\prime} a} \\
& \left.\cdot \operatorname{Im}\left[\bar{R} \mathrm{e}^{2 \mathrm{i} k_{0} v_{s}^{\prime \prime} a}\left(\frac{1-\mathrm{e}^{-2 \mathrm{i} k_{0} v_{s}^{\prime \prime} \xi}}{2 v_{s}^{\prime \prime}}\right)\right]\right\} \mathrm{d} p .
\end{aligned}
$$

Remark 3. One of the quantities we are most interested in studying in this paper is

$$
\|V\|_{H^{1}\left(S_{\xi}\right)}^{2}=\|V\|_{L^{2}\left(S_{\xi}\right)}^{2}+\|\nabla V\|_{L^{2}\left(S_{\xi}\right)}^{2} .
$$

Due to the similarity between the expressions in (30) and (32), without loss of generality we focus on $\|\nabla V\|_{L^{2}\left(S_{\xi}\right)}^{2}$. In particular, our arguments depend heavily on the exponential terms in the integrands in (30) and (32), so the additional terms $\left|v_{s}\right|^{2}$ and $|q|^{2}$ in (30) will have no bearing on our results. 


\section{Properties of $g_{\delta}(p ; \gamma)$}

In this section, we collect some essential properties about the denominator $\left|g_{\delta}\right|^{2}$ in (30). As we will see, the parameter

$$
\gamma \equiv k_{0} a
$$

plays a crucial role in the behavior of the solution $V$ and $E_{\delta}(a)$ in the limit $\delta \rightarrow 0^{+}$.

Lemma 4. Suppose $g_{\delta}$ is defined as in (31). Then for $p \geq 0$ and $\gamma>0$ one has

$$
\begin{aligned}
\lim _{\delta \rightarrow 0^{+}} g_{\delta}(p ; \gamma)= & g_{0}(p ; \gamma) \\
\equiv & \left(\sqrt{p^{2}+1}-\sqrt{p^{2}-1}\right)^{2} \\
& -\left(\sqrt{p^{2}+1}+\sqrt{p^{2}-1}\right)^{2} \mathrm{e}^{-2 \gamma \sqrt{p^{2}+1}}
\end{aligned}
$$

Proof. The result follows from direct calculations since $g_{\delta}$ is a continuous function of $\delta$.

The next lemma plays an essential role in the following discussion.

Lemma 5. Suppose $g_{0}(p ; \gamma)$ is defined as in (35) for $p \geq 0$ and $\gamma>0$. Then there is $\gamma_{*} \approx 0.9373$ such that

(1) if $0<\gamma<\gamma_{*}$, then $g_{0}(p ; \gamma)$ has two distinct real roots of order 1, namely, $1<p_{\gamma}^{1}<p_{\gamma}^{2}$;

(2) if $\gamma>\gamma_{*}$, then $g_{0}(p ; \gamma)$ has no real roots.

We note that $\gamma_{*}$ can be computed as the solution of an optimization problem; more importantly, we emphasize that Lemmas 4 and 5 are independent of the source term $f$ in (4). We will see later that the roots of $g_{0}(p ; \gamma)$ are indicative of anomalous localized resonance. For brevity, we defer the proof of Lemma 5 to the Appendix.

\section{Short Wavelength/High Frequency Regime $\left(\gamma>\gamma_{*}\right)$}

In this section, we prove that, for $\gamma>\gamma_{*}$ (where $\gamma$ was introduced at (34)), $E_{a}(\delta)$ remains bounded as $\delta \rightarrow 0^{+}$for all sources $f \in L^{2}(\mathscr{M})$ with bounded support in $\mathscr{M}$, regardless of how close the source is to the slab. In addition, we also prove that the slab lens behaves as a "shield" in the sense that the solution to the left of the lens, that is, $V_{c}$, is vanishingly small in the limit $\delta \rightarrow 0^{+}$.

3.1. $E_{\delta}(a)$ for $\gamma>\gamma_{*}$. From (30), we have

$$
E_{\delta}(a)=\int_{0}^{\infty} L_{\delta}(p ; \gamma) \mathrm{d} p
$$

where, for $\delta>0, p \geq 0$, and $\gamma>0$,

$$
L_{\delta}(p ; \gamma) \equiv \frac{\left|I_{p}\right|^{2} \mathrm{e}^{2 \gamma v_{m}^{\prime}}}{\left|g_{\delta}(p ; \gamma)\right|^{2}} M_{\delta}(p ; \gamma),
$$

$$
\begin{aligned}
& M_{\delta}(p ; \gamma) \equiv \frac{1+\delta^{2}}{\pi}\left|\nu_{s}-(1+\mathrm{i} \delta) v_{m}\right|^{2}\left\{\left(\left|v_{s}\right|^{2}+p^{2}\right)\right. \\
& {\left[\left(\frac{1-\mathrm{e}^{-2 \gamma v_{s}^{\prime}}}{2 v_{s}^{\prime}}\right)+|R|^{2} \mathrm{e}^{-2 \gamma v_{s}^{\prime}}\left(\frac{1-\mathrm{e}^{-2 \gamma v_{s}^{\prime}}}{2 v_{s}^{\prime}}\right)\right]} \\
& +2\left(-\left|v_{s}\right|^{2}+p^{2}\right) \mathrm{e}^{-2 \gamma v_{s}^{\prime}} \\
& \left.\cdot \operatorname{Im}\left[\bar{R} \mathrm{e}^{2 \mathrm{i} \gamma v_{s}^{\prime \prime}}\left(\frac{1-\mathrm{e}^{-2 \mathrm{i} \gamma v_{s}^{\prime \prime}}}{2 v_{s}^{\prime \prime}}\right)\right]\right\} .
\end{aligned}
$$

We now state the main theorem from this section.

Theorem 6. Suppose $\gamma>\gamma_{*}$ (where $\gamma_{*}$ is introduced in Lemma 5). If there is a constant $C>0$ such that

$$
\left|I_{p}\right| \leq \begin{cases}C & \text { for } 0 \leq p \leq 1, \\ C \mathrm{e}^{-\gamma\left(d_{0} / a\right) \sqrt{p^{2}-1}} & \text { for } 1 \leq p<\infty,\end{cases}
$$

then there is a constant $C_{\gamma}>0$ and $\delta_{\gamma}>0$ such that $\|V\|_{H^{1}\left(S_{a}\right)} \leq C_{\gamma}$ as for all $\delta \leq \delta_{\gamma}$.

The proof of this theorem is somewhat tedious and may be found in the Appendix-although we only prove the theorem for $\|\nabla V\|_{L^{2}\left(S_{a}\right)}^{2}$, Remark 3 implies that it holds for $\|V\|_{L^{2}\left(S_{a}\right)}$ as well. In the next lemma, we show that bound (39) holds for very general sources $f$.

Lemma 7. Suppose $f \in L^{2}(\mathscr{M})$ with compact support; then (39) holds.

Proof. For $0 \leq p \leq 1$, recall from (24) that

$$
I_{p}=\int_{d_{0}}^{d_{1}} \hat{f}\left(s, k_{0} p\right) \mathrm{e}^{-\mathrm{i} k_{0} \sqrt{1-p^{2}} s} \mathrm{~d} s .
$$

Then the triangle, Cauchy-Schwarz, and Jensen inequalities imply that

$$
\begin{aligned}
\left|I_{p}\right| & \leq \int_{d_{0}}^{d_{1}}\left|\hat{f}\left(s, k_{0} p\right)\right| \mathrm{d} s \\
& \leq\left(d_{1}-d_{0}\right)^{1 / 2}\left[\int_{d_{0}}^{d_{1}}\left|\widehat{f}\left(s, k_{0} p\right)\right|^{2} \mathrm{~d} s\right]^{1 / 2} \\
& =\left(d_{1}-d_{0}\right)^{1 / 2}\left[\int_{d_{0}}^{d_{1}}\left|\int_{-\infty}^{\infty} f(s, y) \mathrm{e}^{-\mathrm{i} k_{0} p y} \mathrm{~d} y\right|^{2} \mathrm{~d} s\right]^{1 / 2} \\
& \leq\left(d_{1}-d_{0}\right)^{1 / 2}\left[\int_{d_{0}}^{d_{1}} \int_{-\infty}^{\infty}|f(s, y)|^{2} \mathrm{~d} y \mathrm{~d} s\right]^{1 / 2} \\
& =\left(d_{1}-d_{0}\right)^{1 / 2}\|f\|_{L^{2}(\mathscr{M})} \cdot
\end{aligned}
$$

Similarly, for $p \geq 1$, recall from (24) that

$$
I_{p}=\int_{d_{0}}^{d_{1}} \hat{f}\left(s, k_{0} p\right) \mathrm{e}^{-k_{0} \sqrt{p^{2}-1} s} \mathrm{~d} s .
$$


Then

$$
\begin{aligned}
\left|I_{p}\right| & \leq \int_{d_{0}}^{d_{1}}\left|\widehat{f}\left(s, k_{0} p\right)\right| \mathrm{e}^{-k_{0} \sqrt{p^{2}-1} s} \mathrm{~d} s \\
& \leq\left(d_{1}-d_{0}\right)^{1 / 2}\left[\int_{d_{0}}^{d_{1}}\left|\widehat{f}\left(s, k_{0} p\right)\right|^{2} \mathrm{e}^{-2 k_{0} \sqrt{p^{2}-1} s} \mathrm{~d} s\right]^{1 / 2} \\
& \leq\left(d_{1}-d_{0}\right)^{1 / 2}\|f\|_{L^{2}(\mathscr{C})} \mathrm{e}^{-k_{0} d_{0} \sqrt{p^{2}-1}} \\
& =\left(d_{1}-d_{0}\right)^{1 / 2}\|f\|_{L^{2}(\mathscr{M})} \mathrm{e}^{-\gamma\left(d_{0} / a\right) \sqrt{p^{2}-1}} .
\end{aligned}
$$

To complete the proof, we define $C \equiv\left(d_{1}-d_{0}\right)^{1 / 2}\|f\|_{L^{2}(\mathscr{M})}$.

3.2. Shielding Effect for Large $\gamma$. It turns out that the slab lens behaves as a shield and acts as an almost perfect reflector. This fact was also observed in [21] where it was explained based on the fact that, at least in the lossless nonmagnetic case $\epsilon=-1$, $\mu=1$ will give a purely imaginary wavenumber inside the slab and thus no propagation beyond the slab in region $C$. We have the following.

Theorem 8. Suppose $\gamma \geq 2 \gamma_{*},\left|I_{p}\right|$ satisfies (39), and choose $0<\eta<1$; then there is a constant $C_{\eta}>0$ such that

$$
\left|V_{c}(x, y)\right| \leq C_{\eta} \mathrm{e}^{-\eta \gamma} \quad \forall(x, y) \in \mathscr{C} .
$$

In particular,

$$
\left|V_{c}(x, y)\right| \longrightarrow 0 \text { as } k_{0} \longrightarrow \infty \quad \forall(x, y) \in \mathscr{C}
$$

Remark 9. Lemma 7 implies that Theorems 6 and 8 hold for all sources $f \in L^{2}(\mathscr{M})$ with compact support. However, the bound in (39) is stronger than we need. For example, suppose there is a positive, real-valued function $B(p ; \gamma)$ that is continuous for $0 \leq p<\infty$ and $\gamma_{*} \leq \gamma<\infty$. In addition, for every $\epsilon>0$, suppose that

$$
\begin{array}{ll}
\lim _{p \rightarrow \infty} B(p ; \gamma) \mathrm{e}^{-\epsilon \gamma \sqrt{p^{2}-1}}=0 & \forall \gamma \geq \gamma_{*}, \\
\lim _{\gamma \rightarrow \infty} B(p ; \gamma) \mathrm{e}^{-\epsilon \gamma \sqrt{p^{2}-1}}=0 & \forall p \geq 1 .
\end{array}
$$

For example, if $B(p ; \gamma)$ is a continuous function of $p$ and $\gamma$ that is of polynomial order for $p \rightarrow \infty$ and $\gamma \rightarrow \infty$, it will satisfy (46). Finally, suppose

$$
\left|I_{p}\right| \leq \begin{cases}B(p ; \gamma) & \text { for } 0 \leq p \leq 1, \\ B(p ; \gamma) \mathrm{e}^{-\gamma\left(d_{0} / a\right) \sqrt{p^{2}-1}} & \text { for } 1 \leq p<\infty .\end{cases}
$$

Then, by appropriately modifying (A.72)-(A.75), one can prove that the result of Theorem 6 will hold for sources satisfying (47). Similarly, by appropriately modifying (A.82)(A.86), one can show that Theorem 8 also holds for sources satisfying (47) as long as we replace (44) by

$$
\left|V_{c}(x, y)\right| \leq C_{\eta} \mathrm{e}^{-(\eta-\epsilon) \gamma},
$$

where $0<\epsilon<\eta$.

In particular, certain distributional sources such as dipoles and quadrupoles satisfy (47)-see Section A.2 for more details.

In Figure 2, we plot the solution $V$ to (4) in the case where $f$ is a dipole with dipole moment $[1,0]^{T}, \delta=10^{-12}$, and $\gamma=4 \gamma_{*}$ (we take $a=1$ in all figures throughout the paper). In Figures 2(a) and 2(b), the dipole is located at the point $\left(d_{0}, 0\right)=(4 a, 0)$; in Figures 2(c) and 2(d), the dipole is located closer to the slab at the point $\left(d_{0}, 0\right)=(1.2 a, 0)$. The solution $V$ is smooth throughout the domain; in addition, we observe the "shielding effect" from Theorem 8 in the region to the left of the lens.

In Figure 3, we plot $E_{\delta}(a)$ as a function of various parameters for a dipole source $f$. The parameters we used are in the ranges $10^{-12} \leq \delta \leq 10^{-10}, 1.01 \gamma_{*} \leq \gamma \leq 2 \gamma_{*}$, and $1.2 a \leq d_{0} \leq 2 a$. We note that $E_{\delta}(a)$ depends strongly on $\delta, \gamma$, and $d_{0}$, but, because $\gamma>\gamma_{*}, E_{\delta}(a)$ is quite small.

Figure 4 is similar to Figure 2, except in Figure 4 we take

$$
\begin{aligned}
& f(x, y) \\
& =\left\{\begin{array}{l}
C\left[\frac{2}{d_{1}-d_{0}}\left(x-d_{0}\right)-1\right]^{3}\left[\left|\frac{2}{d_{1}-d_{0}}\left(x-d_{0}\right)-1\right|-1\right]^{3} \cdot\left[\frac{2}{h_{1}-h_{0}}\left(y-h_{0}\right)-1\right]^{3}\left[\left|\frac{2}{h_{1}-h_{0}}\left(y-d_{0}\right)-1\right|-1\right]^{3} \text { for } d_{0} \leq x \leq d_{1}, h_{0} \leq y \leq h_{1} \\
0
\end{array}\right. \\
& \text { otherwise. }
\end{aligned}
$$

Although this source has compact support, in contrast to the dipolar source considered above it is in $L^{2}(\mathscr{M})$ and is twice continuously differentiable; we chose this source to emphasize that the ideas presented in this paper do not rely on the extreme nature of distributional sources such as dipoles.

To construct the plots, we have taken $C=10^{4}, h_{1}=$ $-h_{0}=1$, and $d_{1}=d_{0}+2$. The solution $V$ is smooth throughout 


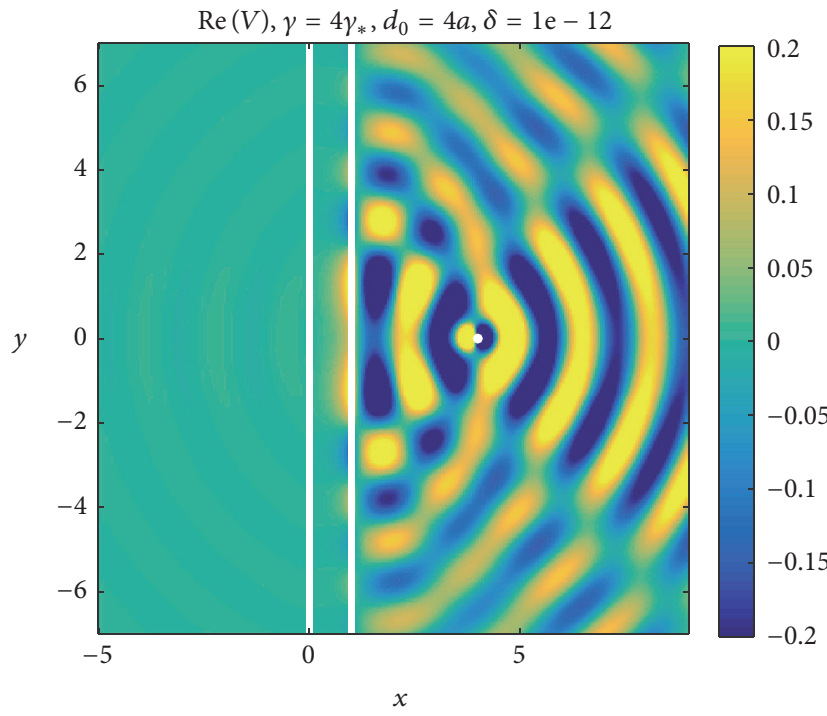

(a)

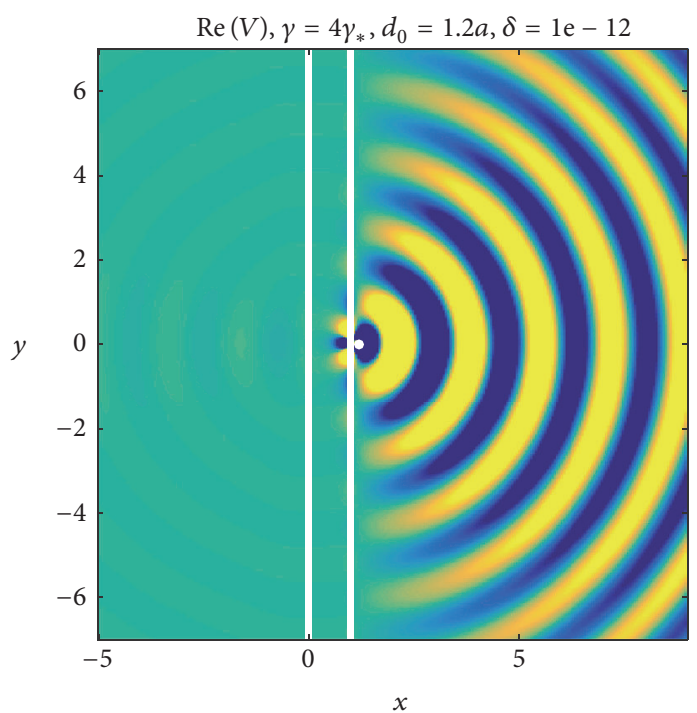

(c)

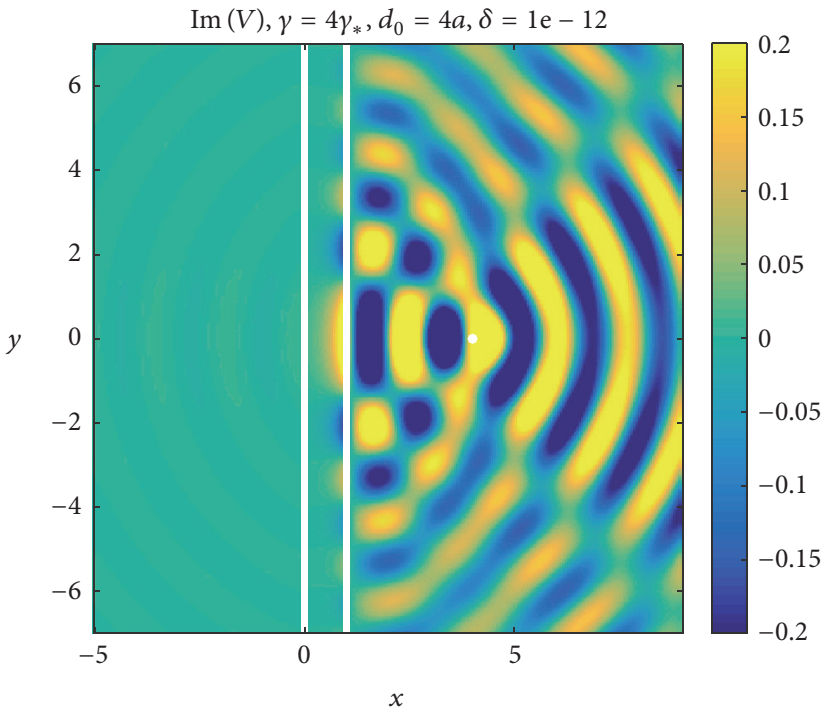

(b)

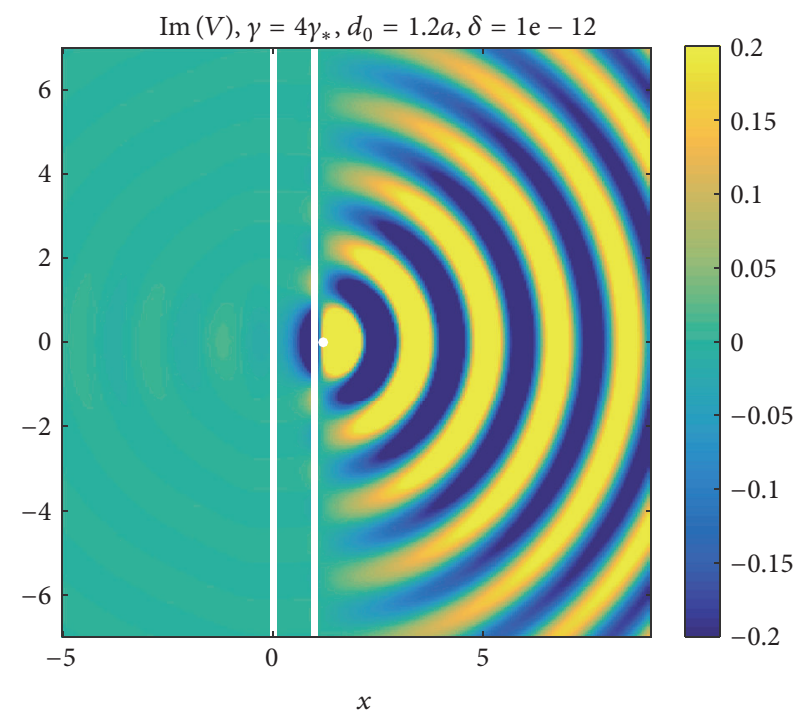

(d)

Figure 2: This is a plot of $V$, the solution to (4), when $f$ is a dipole and $\gamma=2 \gamma_{*}$ : (a) $\operatorname{Re}(V)$ and (b) $\operatorname{Im}(V)$ for $d_{0}=4 a$; (c) $\operatorname{Re}(V)$ and (d) $\operatorname{Im}(V)$ for $d_{0}=1.2 a$. To make the behavior of $V$ clearer, we clipped the maximum and minimum values in each plot to 0.2 (yellow) and -0.2 (blue), respectively.

the domain and very small in the region to the left of the slab.

\section{Long-Wavelength/Low Frequency Regime $\left(\gamma<\gamma_{*}\right)$}

Unfortunately, the complicated nature of expression (30) has thus far prevented us from deriving lower bounds on $E_{\delta}(a)$ that would allow us to prove that $E_{\delta}(a) \rightarrow \infty$ as $\delta \rightarrow 0^{+}$. Undaunted, in this section we present an heuristic argument, coupled with numerical experiments, to illustrate why we believe the slab lens under consideration exhibits ALR in the long-wavelength regime.

4.1. Blow-Up of $E_{\delta}(a)$. The key result of this section is Lemma 5: $\left|g_{0}(p ; \gamma)\right|$ has two real roots when $\gamma<\gamma_{*}$; namely, $1<p_{\gamma}^{1}<p_{\gamma}^{2}$. Because both roots are larger than 1 , the main contribution to the blow-up of $E_{\delta}(a)$ comes from the integral over the interval $1 \leq p<\infty$. Indeed, the following lemma shows that we do not need to worry about the integral over the interval $0 \leq p \leq 1$.

Lemma 10. Suppose $0<\gamma \leq \gamma_{*}$ and $f \in L^{2}(\mathscr{M})$ with compact support. Then there is a positive constant $C_{\gamma}$ and $\delta_{\gamma}>0$ such that

$$
\int_{0}^{1} L_{\delta}(p ; \gamma) \mathrm{d} p=\int_{0}^{1} \frac{\left|I_{p}\right|^{2}}{\left|g_{\delta}\right|^{2}} M_{\delta}(p ; \gamma) \mathrm{d} p \leq C_{\gamma}
$$

for all $0<\delta \leq \delta_{\gamma}$ 


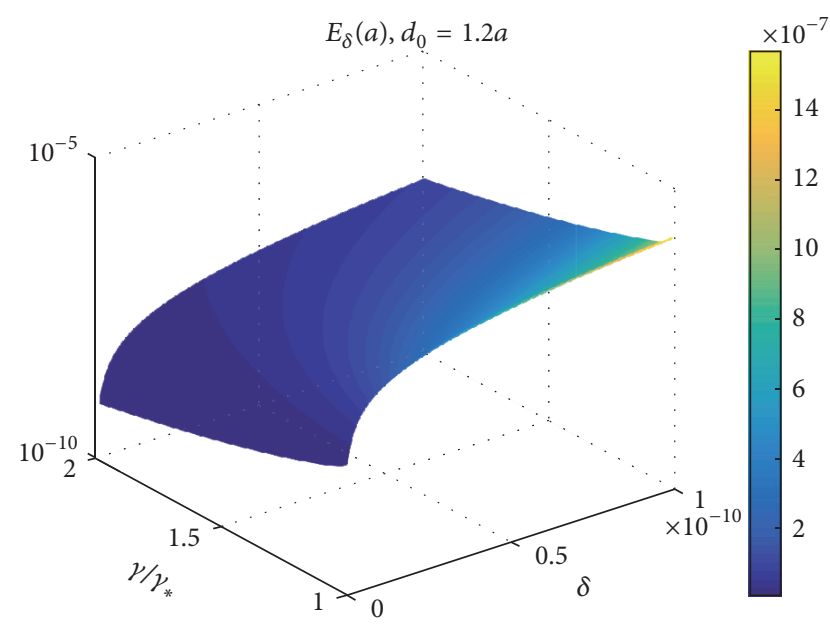

(a)

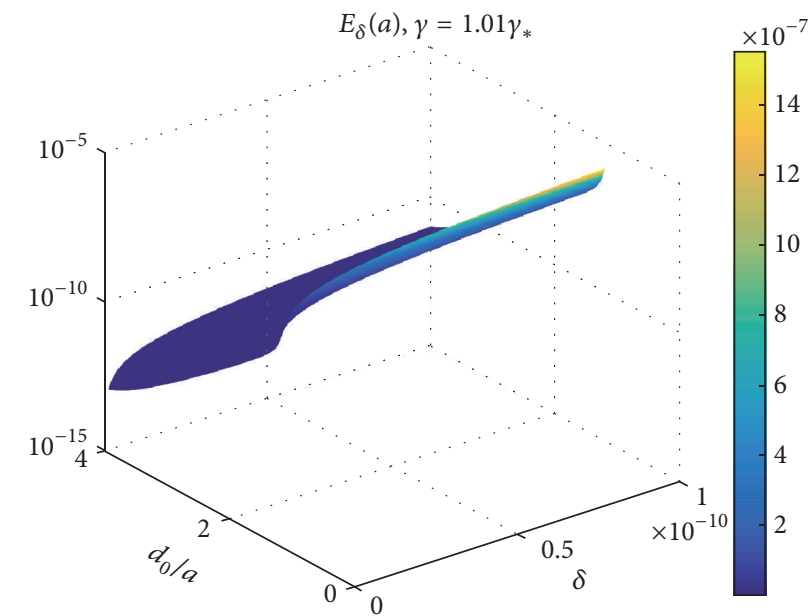

(c)

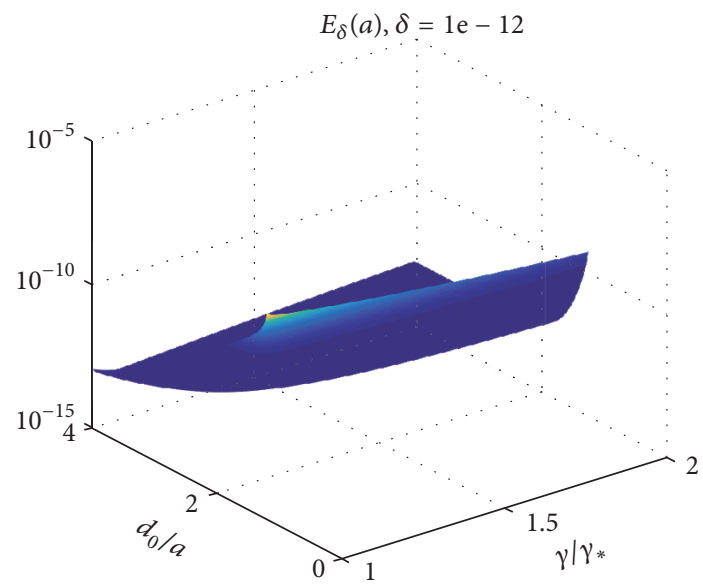

(e)

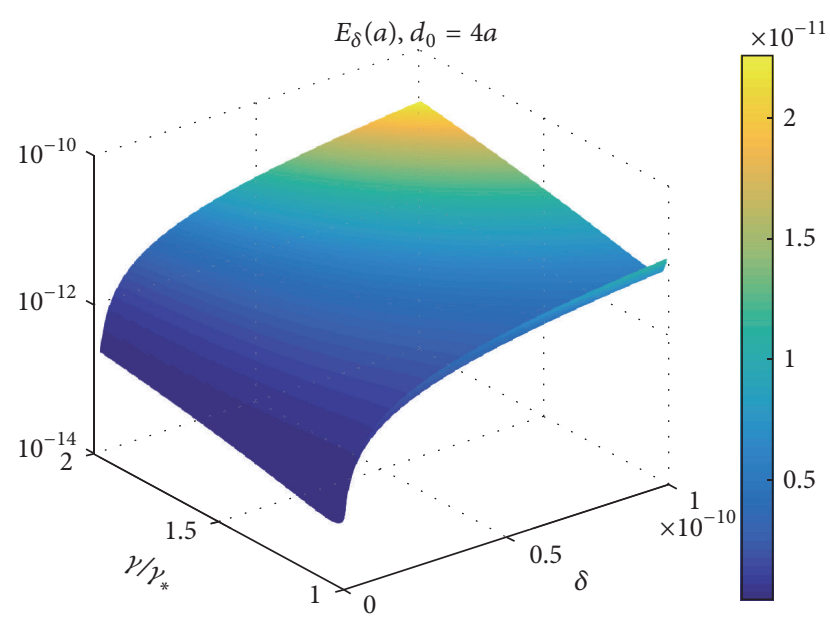

(b)

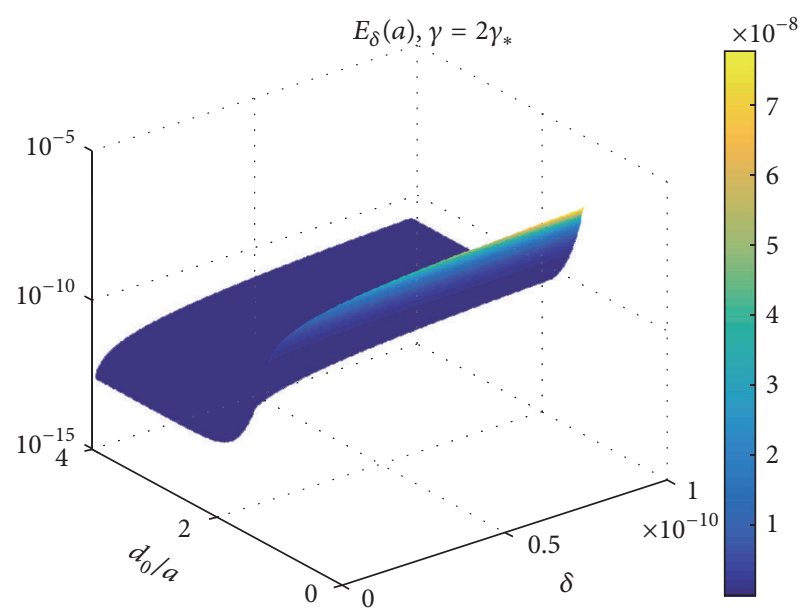

(d)
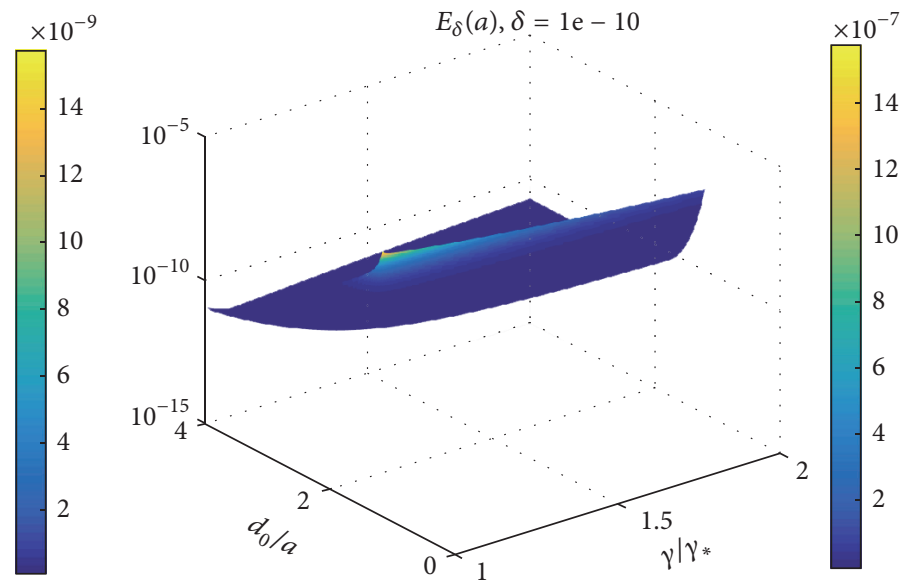

(f)

Figure 3: These are plots of $E_{\delta}(a)$ as a function of (a) $\delta$ and $\gamma\left(d_{0}=1.2 a\right)$; (b) $\delta$ and $\gamma\left(d_{0}=4 a\right)$; (c) $\delta$ and $d_{0}\left(\gamma=1.01 \gamma_{*}\right)$; (d) $\delta$ and $d_{0}$ $\left(\gamma=2 \gamma_{*}\right)$; (e) $\gamma$ and $d_{0}\left(\delta=10^{-10}\right)$; (f) $\gamma$ and $d_{0}\left(\delta=10^{-12}\right)$. 


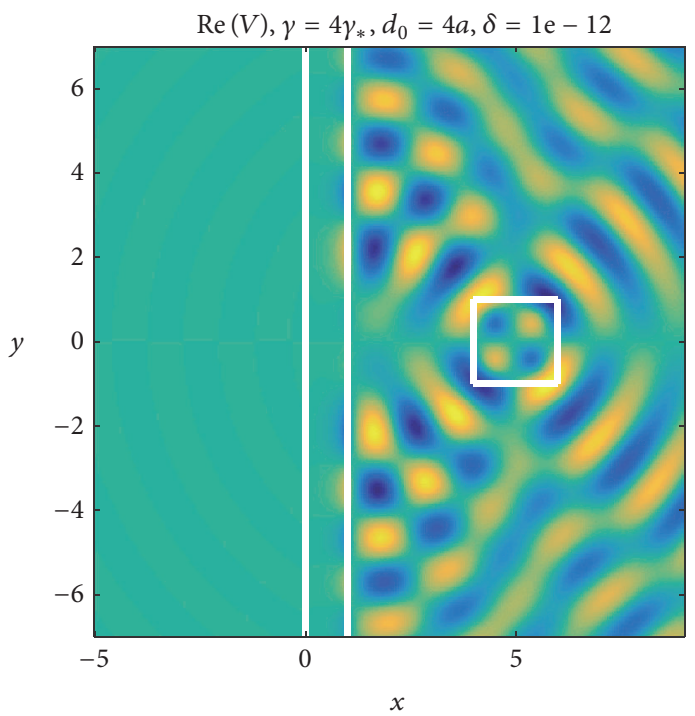

(a)

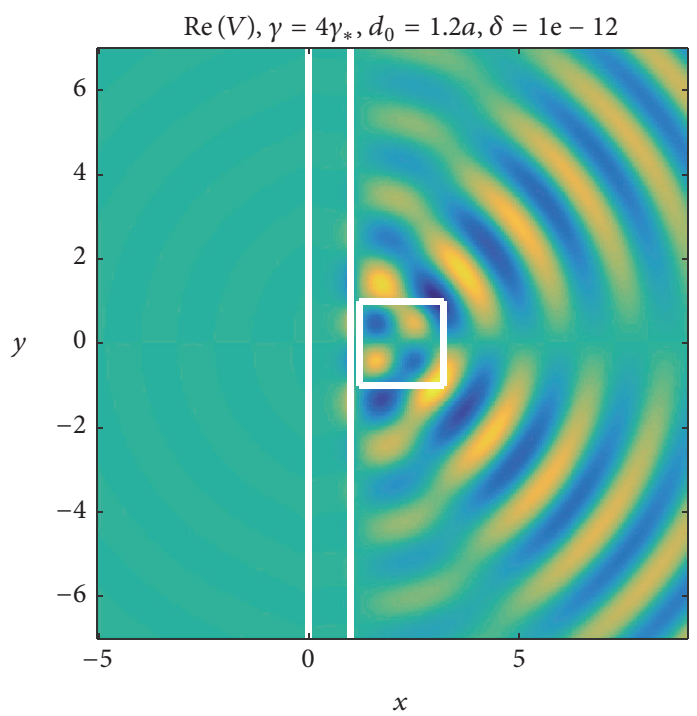

(c)
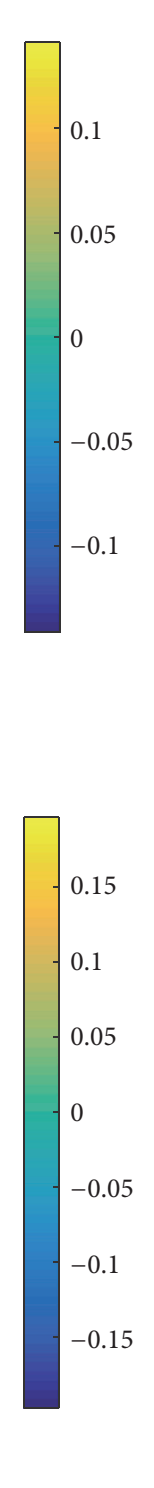

Figure 4: This is a plot of $V$, the solution to (4), when $f$ is the function in (49) and $\gamma=2 \gamma_{*}$ : (a) $\operatorname{Re}(V)$ and (b) $\operatorname{Im}(V)$ for $d_{0}=4 a$; (c) $\operatorname{Re}(V)$ and $(\mathrm{d}) \operatorname{Im}(V)$ for $d_{0}=1.2 a$. To make the behavior of $V$ clearer, we clipped the maximum and minimum values in each plot to 0.2 (yellow) and -0.2 (blue), respectively.

Remark 11. We emphasize that Lemma 10 also holds for those sources for which the bound in (47) holds (e.g., dipole sources)-see Remark 9.

Proof. First, we note that $M_{\delta}(p ; \gamma)$ is continuous for $\delta \in$ $[0,1], p \in[0,1]$, and $\gamma \in\left[0, \gamma_{*}\right]$, so it is bounded by a constant independent of $\delta$, $p$, and $\gamma$. Additionally, $\left|I_{p}\right|^{2}$ is also bounded by a constant, thanks to Lemma 7 . All that remains for us to show is that $\left|g_{\delta}(p ; \gamma)\right|$ is bounded away from 0 .

We define the function

$$
\Xi_{\delta}(\gamma) \equiv \max _{p \in[0,1]}\left\|g_{\delta}(p ; \gamma)|-| g_{0}(p ; \gamma)\right\|
$$

Because $\left|g_{\delta}(p ; \gamma)\right|$ and $\left|g_{0}(p ; \gamma)\right|$ are both continuous for $0 \leq$ $p \leq 1$, the above maximum is attained, say at $p=p_{\delta}^{*}(\gamma)$. This means that

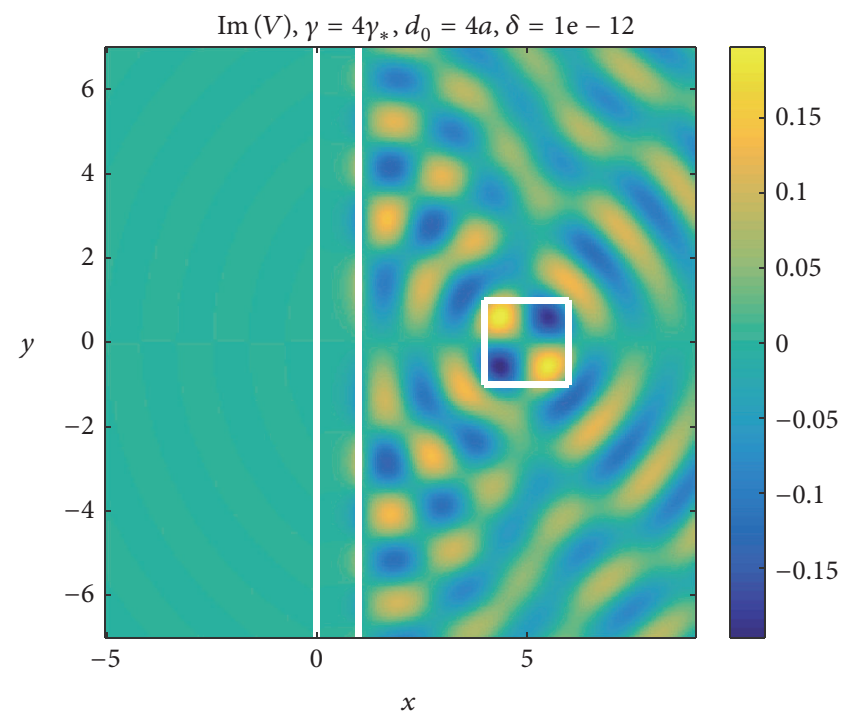

(b)

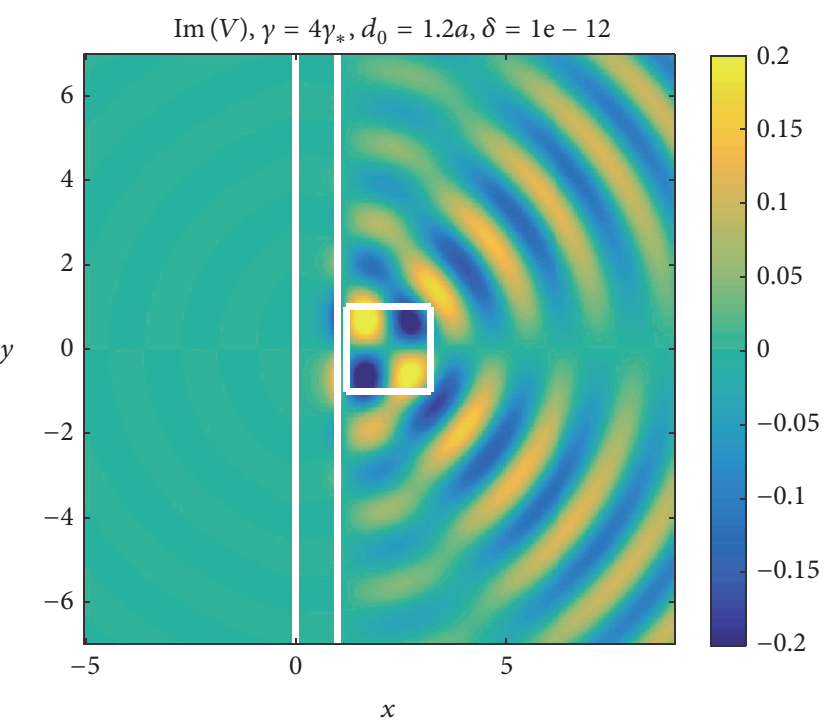

(d)

$$
\Xi_{\delta}(\gamma)=\left\|g\left(p_{\delta}^{*}(\gamma) ; \gamma\right)|-| g_{0}\left(p_{\delta}^{*}(\gamma) ; \gamma\right)\right\| .
$$

Now let $\left\{\delta_{n}\right\}_{n=1}^{\infty}$ be a sequence converging to 0 as $n \rightarrow$ $\infty$. Because $p_{\delta_{n}}^{*}(\gamma)$ is a bounded sequence, it has a convergent subsequence $p_{\delta_{n_{k}}^{*}}^{*}(\gamma)$. Along this subsequence,

$$
\begin{aligned}
\Xi_{\delta_{n_{k}}}(\gamma)=\left\|g_{\delta_{n_{k}}}\left(p_{\delta_{n_{k}}}^{*}(\gamma) ; \gamma\right)|-| g_{0}\left(p_{\delta_{n_{k}}}^{*}(\gamma) ; \gamma\right)\right\| & \longrightarrow 0 \\
\text { as } k & \longrightarrow \infty
\end{aligned}
$$

by Lemma 4 . In other words, every sequence $\Xi_{\delta_{n}}(\gamma)$ has a subsequence that converges to 0 , which implies that every sequence $\Xi_{\delta_{n}}$ converges to 0 . Because the original sequence $\delta_{n}$ was arbitrary, this implies that

$$
\lim _{\delta \rightarrow 0^{+}} \Xi_{\delta}(\gamma)=0
$$


In combination with (51), this implies that $\left|g_{\delta}(p ; \gamma)\right|$ converges to $\left|g_{0}(p ; \gamma)\right|$ uniformly in $p$ for $0 \leq p \leq 1$. Thus for every $\epsilon>0$ there is $\delta_{\gamma}>0$ such that

$$
\left|g_{\delta}(p ; \gamma)\right| \geq\left|g_{0}(p ; \gamma)\right|-\epsilon
$$

for all $p \in[0,1]$ and all $0<\delta \leq \delta_{\gamma}$. If we take

$$
\epsilon=\frac{1}{2} \min _{0 \leq p \leq 1}\left|g_{0}(p ; \gamma)\right|
$$

then

$$
\left|g_{\delta}(p ; \gamma)\right| \geq \frac{1}{2}\left|g_{0}(p ; \gamma)\right| \geq C_{\gamma}>0
$$

for all $p \in[0,1]$ (the last two inequalities hold because the roots of $\left|g_{0}\right|$ are larger than 1 by Lemma 5). Combining this result with the first paragraph of the proof gives us the bound

$$
\begin{aligned}
& \int_{0}^{1} \frac{\left|I_{p}\right|^{2}}{\left|g_{\delta}(p ; \gamma)\right|^{2}} M_{\delta}(p ; \gamma) \mathrm{d} p \leq C \int_{0}^{1} \frac{1}{\left|g_{0}(p ; \gamma)\right|^{2}} \mathrm{~d} p \\
& \quad \leq C_{\gamma}
\end{aligned}
$$

for some constant $C_{\gamma}>0$.

The preceding lemma proves that we only need to study the integral in (30) over the interval $1 \leq p<\infty$. Because $\left|g_{\delta}(p ; \gamma)\right| \rightarrow\left|g_{0}(p ; \gamma)\right|$ as $\delta \rightarrow 0^{+}$, it should be the case that $\left|g_{\delta}(p ; \gamma)\right| \approx 0$ near the roots of $\left|g_{0}(p ; \gamma)\right|$. Inspired by our earlier work in the quasistatic regime, we conjecture that $\left|g_{\delta}\left(p_{\gamma}^{1} ; \gamma\right)\right|$ and $\left|g_{\delta}\left(p_{\gamma}^{2} ; \gamma\right)\right|$ are on the order of $\delta$ as $\delta \rightarrow 0^{+}$.

Conjecture 12. Suppose $0<\gamma<\gamma_{*}$, and let $1<p_{\gamma}^{1}<p_{\gamma}^{2}$ be the roots of $g_{0}(p ; \gamma)$. Then there is $\delta_{\gamma}>0$ such that $\left|g_{\delta}(p ; \gamma)\right| \neq$ 0 for all $1 \leq p<\infty$ and all $0<\delta \leq \delta_{\gamma}$; however, $\left|g_{\delta}\left(p_{\gamma}^{1} ; \gamma\right)\right|=$ $\mathcal{O}(\delta)$ and $\left|g_{\delta}\left(p_{\gamma}^{2} ; \gamma\right)\right|=\mathcal{O}(\delta)$ as $\delta \rightarrow 0^{+}$.

One way to prove this conjecture would be to expand $\left|g_{\delta}\left(p_{\gamma}^{j} ; \gamma\right)\right|$ (for $\left.j=1,2\right)$ in Taylor series around $\delta=0$ and then prove that $\partial\left|g_{\delta}\left(p_{\gamma}^{j} ; \gamma\right)\right| / \partial \delta$ is uniformly bounded for $p \in$ $[1, \infty)$ and $\delta$ small enough. Unfortunately, these derivatives are quite complicated; moreover, numerical experiments indicate that they become unbounded as $p \rightarrow \infty$, so it is unlikely that this technique would work even if the expressions were suitable for analytic study. To provide some justification for Conjecture 12, in Figures 5(a) and 5(b) we plot

$$
\begin{aligned}
& \frac{\left|g_{\delta}\left(p_{\gamma}^{1} ; \gamma\right)\right|}{\delta} \\
& \frac{\left|g_{\delta}\left(p_{\gamma}^{1} ; \gamma\right)\right|}{\delta}
\end{aligned}
$$

as functions of $\delta$ and $\gamma$ over the ranges $10^{-12} \leq \delta \leq 10^{-10}$ and $0.1 \gamma_{*} \leq \gamma \leq 0.99 \gamma_{*}$ (we believe the functions in (59) remain bounded as $\delta \rightarrow 0$ for all $0<\gamma<\gamma_{*}$; however, $p_{\gamma}^{2} \rightarrow \infty$ as $\gamma \rightarrow 0$, so the numerical computation of the roots becomes more difficult as $\gamma$ gets closer to 0 . Similarly, $p_{\gamma_{*}}^{1}=p_{\gamma_{*}}^{2}$, so as $\gamma$ gets close to $\gamma_{*}$ it becomes difficult to distinguish the roots). For each $\gamma$, we see that the functions in (59) remain bounded as $\delta$ gets close to 0 , which seems to indicate that $\left|g_{\delta}\left(p_{\gamma}^{1} ; \gamma\right)\right|=\mathcal{O}(\delta)$ and $\left|g_{\delta}\left(p_{\gamma}^{2} ; \gamma\right)\right|=\mathcal{O}(\delta)$ as $\delta \rightarrow 0$. Curiously, both functions in (59) seem to depend very weakly on $\delta$.

Next, we conjecture that the $\mathscr{O}(\delta)$ behavior of $\left|g_{\delta}(p ; \gamma)\right|$ near $p_{\gamma}^{1}$ and $p_{\gamma}^{2}$ is not canceled by the term $M_{\delta}(p ; \gamma)$ in the numerator.

Conjecture 13. Suppose $0<\gamma<\gamma_{*}$, and define $M_{\delta}(p ; \gamma)$ as in (38). Then there exist positive constants $\delta_{\gamma}$ and $C_{\gamma}$ such that $M_{\delta}(p ; \gamma) \geq C_{\gamma}$ near $p_{\gamma}^{1}$ and $p_{\gamma}^{2}$ for all $0<\delta \leq \delta_{\gamma}$.

If Conjectures 12 and 13 are true, then (36)-(37) imply that the part of the integrand $L_{\delta}(p ; \gamma)$ that is independent of the source $f$, namely,

$$
\mathrm{e}^{2 \gamma v_{m}^{\prime}} \frac{M_{\delta}(p ; \gamma)}{\left|g_{\delta}(p ; \gamma)\right|^{2}},
$$

is on the order of $\delta^{-2}$ near $p_{\gamma}^{1}$ and $p_{\gamma}^{2}$ as $\delta \rightarrow 0^{+}$. If $\left|I_{p}\right|^{2}$ is also bounded away from 0 near $p_{\gamma}^{1}$ and $p_{\gamma}^{2}$, the entire integrand $L_{\delta}(p ; \gamma)$ will have values on the order of $\delta^{-2}$ near $p_{\gamma}^{1}$ and $p_{\gamma}^{2}$.

To provide some justification for Conjecture 13, in Figures 6(a) and 6(b) we plot

$$
\begin{aligned}
& M_{\delta}\left(p_{\gamma}^{1} ; \gamma\right), \\
& M_{\delta}\left(p_{\gamma}^{2} ; \gamma\right)
\end{aligned}
$$

as functions of $\delta$ and $\gamma$ over the same intervals as in Figure 5. In particular, we note that $M_{\delta}\left(p_{\gamma}^{1} ; \gamma\right)$ and $M_{\delta}\left(p_{\gamma}^{2} ; \gamma\right)$ are both bounded away from 0 and seem to depend quite weakly on $\delta$.

Finally, to obtain a blow-up in $E_{\delta}(a)$, it should be the case that $\left|I_{p}\right|$ does not conquer the small values of $\left|g_{\delta}\right|$ near $p_{\gamma}^{1}$ and $p_{\gamma}^{2}$. Heuristically, there will be no blow-up if $\left|I_{p}\right| \approx 0$ near $p_{\gamma}^{1}$ and $p_{\gamma}^{2}$. In the next section, we present numerical evidence that suggests that sources with $\left|I_{p_{\gamma}^{1}}\right|=\left|I_{p_{\gamma}^{2}}\right|=0$ do not lead to ALR.

On the other hand, recall from (24) that

$$
I_{p}=\int_{d_{0}}^{d_{1}} \widehat{f}\left(x, k_{0} p\right) \mathrm{e}^{-k_{0} \sqrt{p^{2}-1} s} \mathrm{~d} s .
$$

Again we take our inspiration from the quasistatic case [23, 24]. If $d_{0} \gg a$, then the exponential in the above integrand will be extremely small (especially because $p_{\gamma}^{1}$ and $p_{\gamma}^{2}$ are both greater than 1). In particular, the exponential may be small enough so that it cancels out the effect of the denominator near $p_{\gamma}^{1}$ and $p_{\gamma}^{2}$. We emphasize that this is not rigorous, but we hope that it may provide a starting point for future investigations.

Conjecture 14. Suppose $0<\gamma<\gamma_{*}$. Then there exist sources $f \in L^{2}(\mathscr{M})$ with compact support or distributional sources 


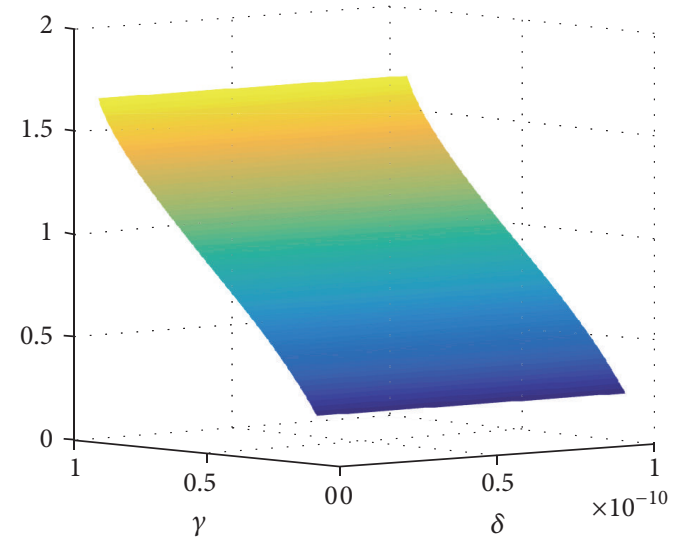

(a)

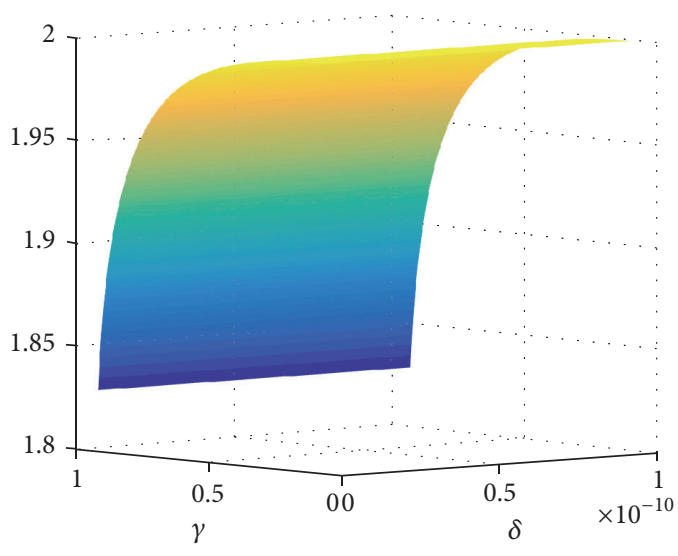

(b)

Figure 5: In this figure, we plot (a) $\left|g_{\delta}\left(p_{\gamma}^{1} ; \gamma\right)\right| / \delta$ and (b) $\left|g_{\delta}\left(p_{\gamma}^{2} ; \gamma\right)\right| / \delta$ over the range $10^{-12} \leq \delta \leq 10^{-10}$ and $0.1 \gamma_{*} \leq \gamma \leq 0.99 \gamma_{*}$.

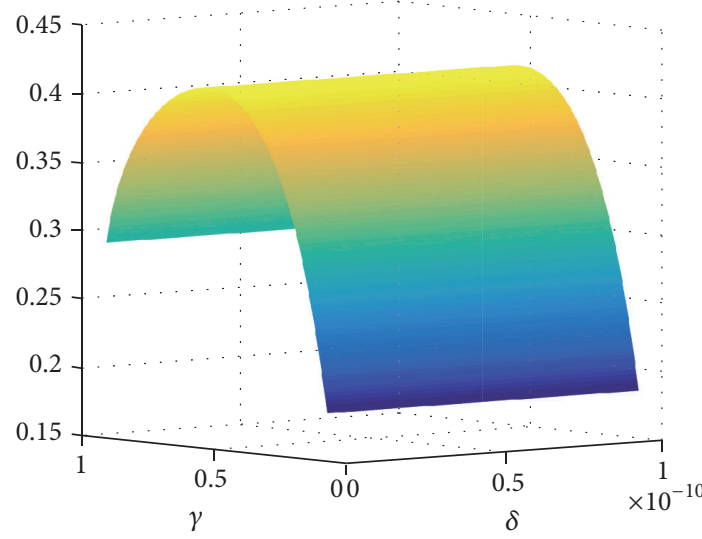

(a)

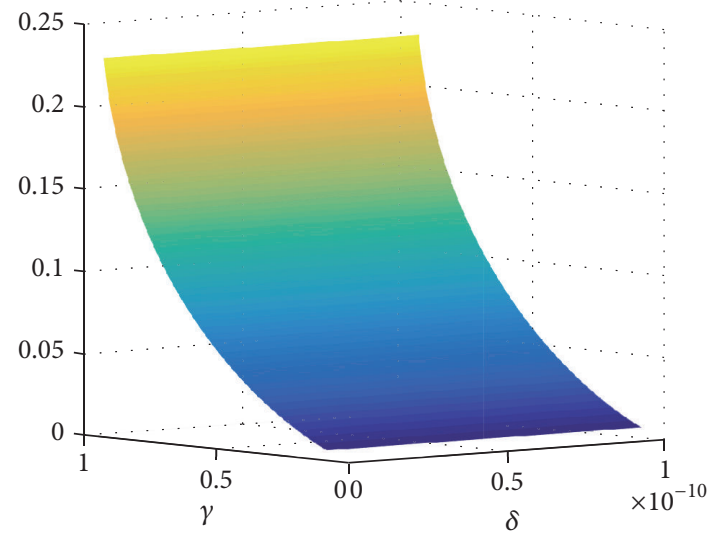

(b)

Figure 6: In this figure, we plot (a) $M_{\delta}\left(p_{\gamma}^{1} ; \gamma\right)$ and (b) $M_{\delta}\left(p_{\gamma}^{2} ; \gamma\right)$ over the range $10^{-12} \leq \delta \leq 10^{-10}$ and $0.1 \gamma_{*} \leq \gamma \leq 0.99 \gamma_{*}$.

such as dipoles such that, for any $0<\xi \leq a, E_{\delta}(\xi) \rightarrow \infty$ if $d_{0}$ is "close enough" to $a$ and $E_{\delta}(\xi) \leq C_{\gamma}$ for some positive constant $C_{\gamma}$ if $d_{0}$ is "far enough away" from a. This critical distance may depend on $\gamma$.

Moreover, there are positive constants $b_{\gamma}, C_{\gamma}$, and $\delta_{\gamma}$ such that, for all $0<\delta \leq \delta_{\gamma}$,

$$
|V(x, y)| \leq C_{\gamma}
$$

for all $(x, y) \in \mathscr{C} \cup \mathscr{M}$ with $|x|>b_{\gamma}$.

Remark 15. If it is only the case that

$$
\limsup _{\delta \rightarrow 0^{+}} E_{\delta}(\xi)=\infty
$$

then we say that weak ALR occurs. Because $E_{\delta}(\xi)$ is difficult to deal with analytically, we cannot say much more on this. It is difficult to determine whether

$$
\begin{aligned}
& \limsup _{\delta \rightarrow 0^{+}} E_{\delta}(\xi)=\infty \\
& \text { or } \lim _{\delta \rightarrow 0^{+}} E_{\delta}(\xi)=\infty
\end{aligned}
$$

using only numerical techniques. In particular, if the limit supremum of $E_{\delta}(\xi)$ is $\infty$, there is at least one sequence $\delta_{n} \rightarrow$ $0^{+}$along which $E_{\delta_{n}}(\xi) \rightarrow \infty$; however, it may be the case that $E_{\delta_{n}}(\xi) \rightarrow \infty$ for all sequences $\delta_{n} \rightarrow 0^{+}$except a few very special sequences that would be extremely difficult to find via numerical experiments alone.

Figures 7 and 8 are exactly the same as Figures 2 and 4 except $\gamma=0.5 \gamma_{*}$ in Figures 7 and 8. In Figures 7(a), 7(b), $8(\mathrm{a})$, and 8(b), the sources (a dipole in Figure 7 and the source $f$ from (49) in Figure 8) are located at $d_{0}=4 a$, and the solution $V$ appears to be smooth throughout the domain. As the sources move closer to the slab, resonant regions appear around both boundaries of the slab at $x=0$ and $x=a$. Figures 7(c), 7(d), 8(c), and 8(d) contain plots of $V$ when $d_{0}=$ 1.2a. From these figures we see that the extreme oscillations of $V$ are contained near the boundaries of the slab and that the boundaries between the resonant and nonresonant regions are sharp and not defined by the boundaries of the slab; away from the slab, $V$ is smooth and bounded. This is highly characteristic of ALR (see, e.g., [1,23] and the references therein). Moreover, Figures 7 and 8 indicate that an image of 


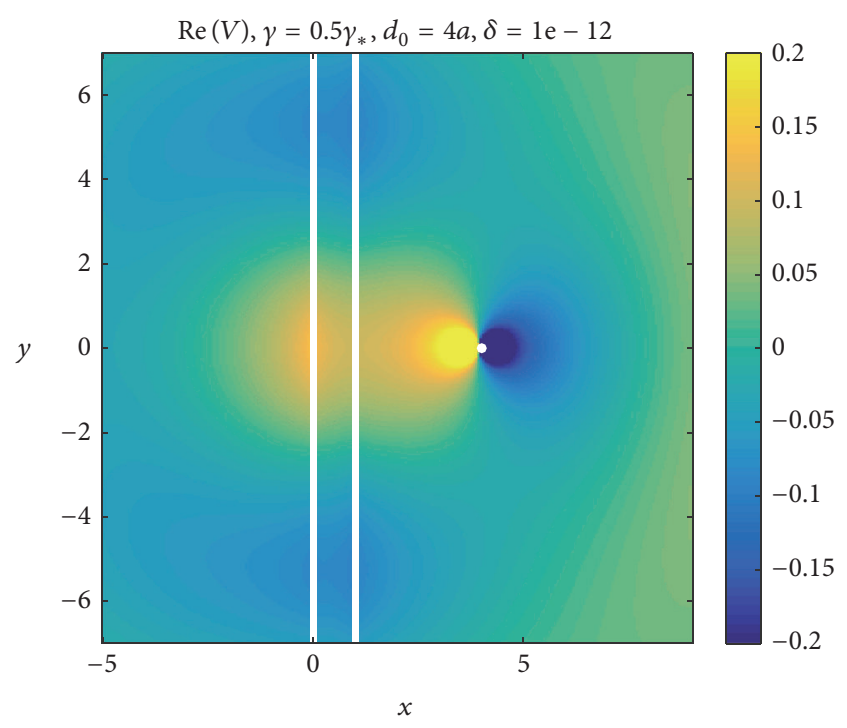

(a)

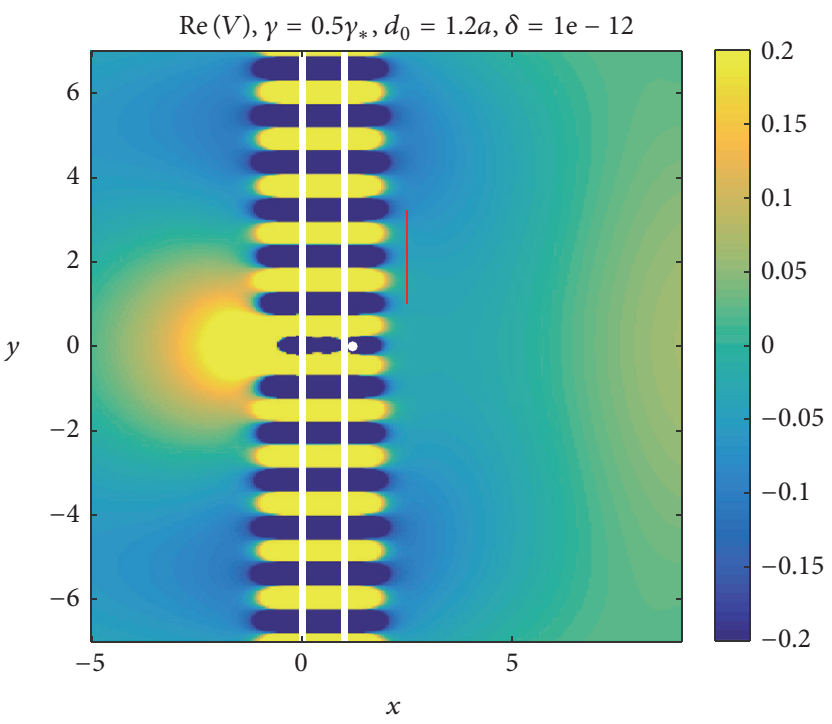

(c)

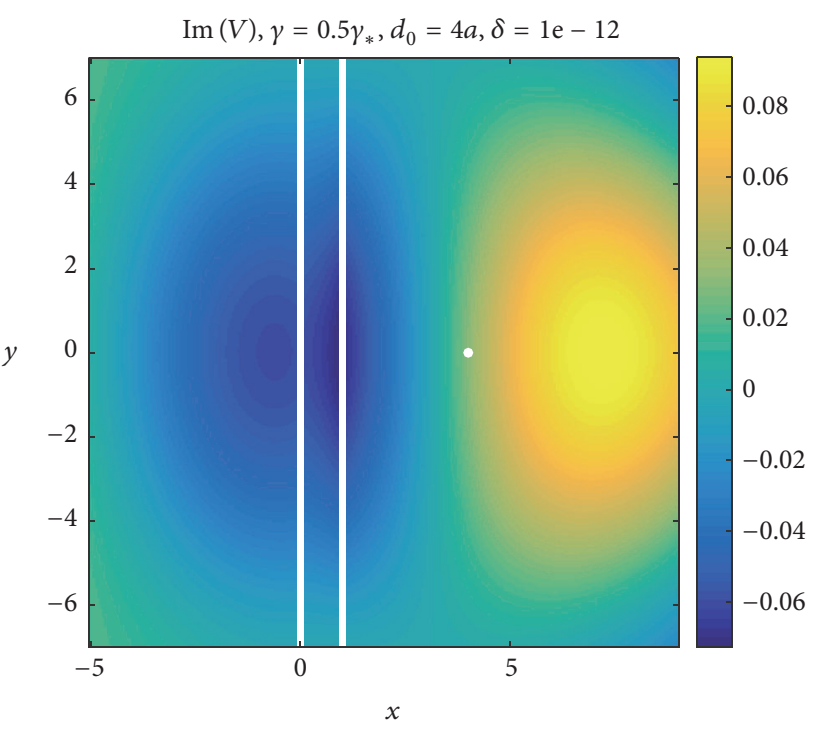

(b)

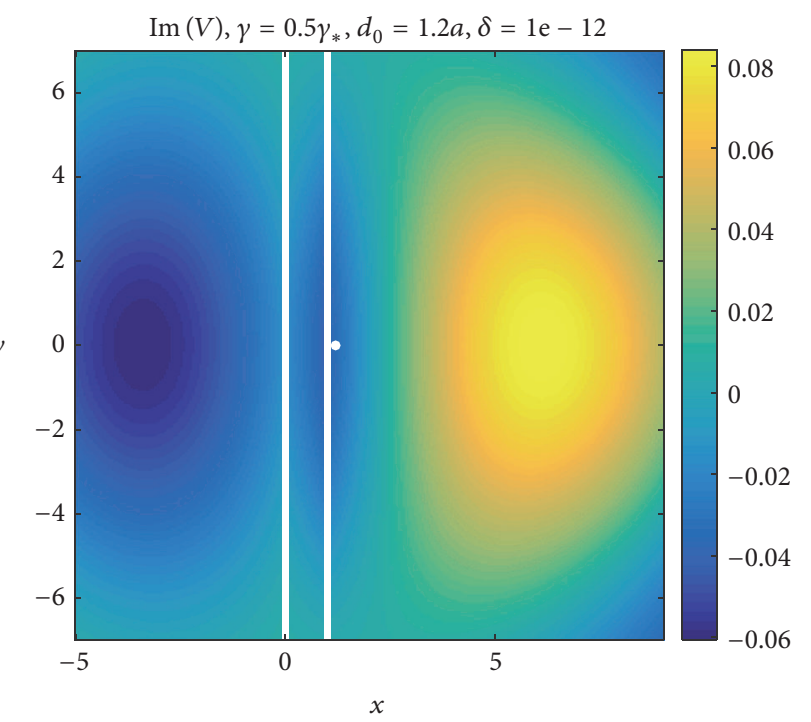

(d)

Figure 7: This is a plot of $V$, the solution to (4), when $f$ is a dipole and $\gamma=0.5 \gamma_{*}$ : (a) $\operatorname{Re}(V)$ and (b) $\operatorname{Im}(V)$ for $d_{0}=4 a$; (c) $\operatorname{Re}(V)$ and (d) $\operatorname{Im}(V)$ for $d_{0}=1.2 a$. To make the behavior of $V$ clearer, we clipped the maximum and minimum values in each plot to 0.2 (yellow) and -0.2 (blue), respectively. The vertical red line in (c) extends a distance of $2 \lambda_{\gamma}$, where $\lambda_{\gamma}$ is defined in (66).

(part of) the solution $V$ is focused in the region to the left of the lens (outside of the resonant region); this is in stark contrast to the high frequency regime illustrated in Figures 2 and 4, in which the solution $V$ in the region to the left of the slab is barely noticeable. Indeed, in the quasistatic regime, ALR is closely associated with this so-called superlensing [1]; since ALR does not occur for $\gamma>\gamma_{*}$ (see Theorem 6), we do not expect to see the superlensing effect in this regime (see Theorem 8).

Figures 7(c) and 8(c) provide an additional insight into Conjecture 14. In general, for $q \approx k_{0} p_{\gamma}^{2}$ (where $p_{\gamma}^{2}$ is the larger root of $\left.g_{0}(p ; \gamma)\right)$, the coefficient $A_{q}$ from (23) becomes very large since its denominator is proportional to $g_{\delta}(p ; \gamma)$ and $g_{\delta}\left(p_{\gamma}^{2} ; \gamma\right) \approx g_{0}\left(p_{\gamma}^{2} ; \gamma\right)=0$ for $\delta$ small enough. Recalling that the Fourier transform variable $q=k_{0} p$ represents a wavenumber in the $y$-direction with corresponding wavelength $\lambda=2 \pi / q$, this implies that the solution $V$ should exhibit prominent oscillations with wavelength on the order of

$$
\lambda_{\gamma}=\frac{2 \pi}{k_{0} p_{\gamma}^{2}} .
$$

In Figures $7(\mathrm{c})$ and $8(\mathrm{c})$, we have drawn a vertical red line of length $2 \lambda_{\gamma}$. This red line covers approximately 2 wavelengths of oscillation in the resonant region, which seems to indicate that at least one of the zeros of $g_{0}$, namely, $p_{\gamma}^{2}$, is responsible for ALR. Because $p_{\gamma}^{2}$ is independent of $f$, the above argument also suggests that the wavelength of the resonant oscillations 


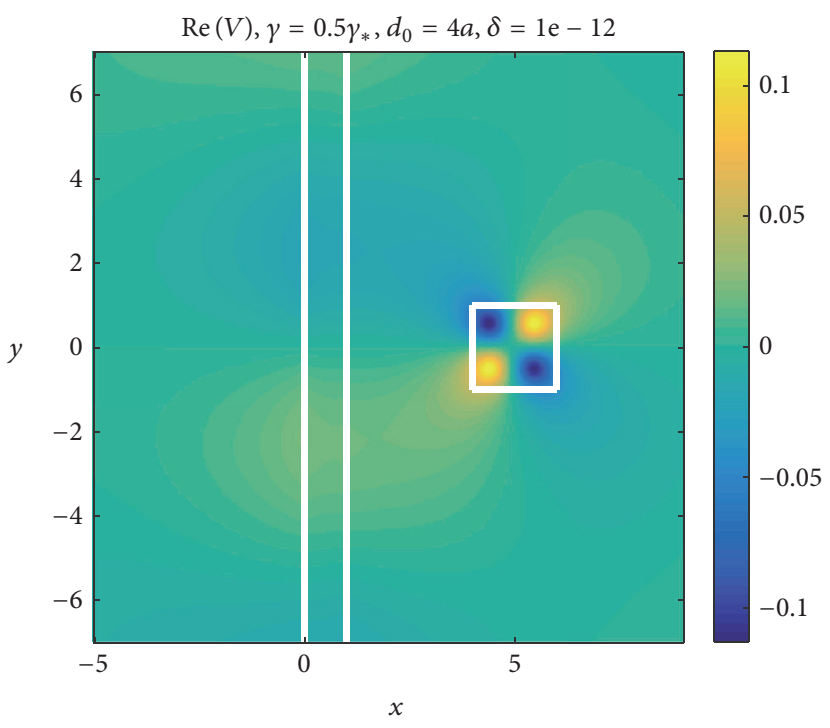

(a)

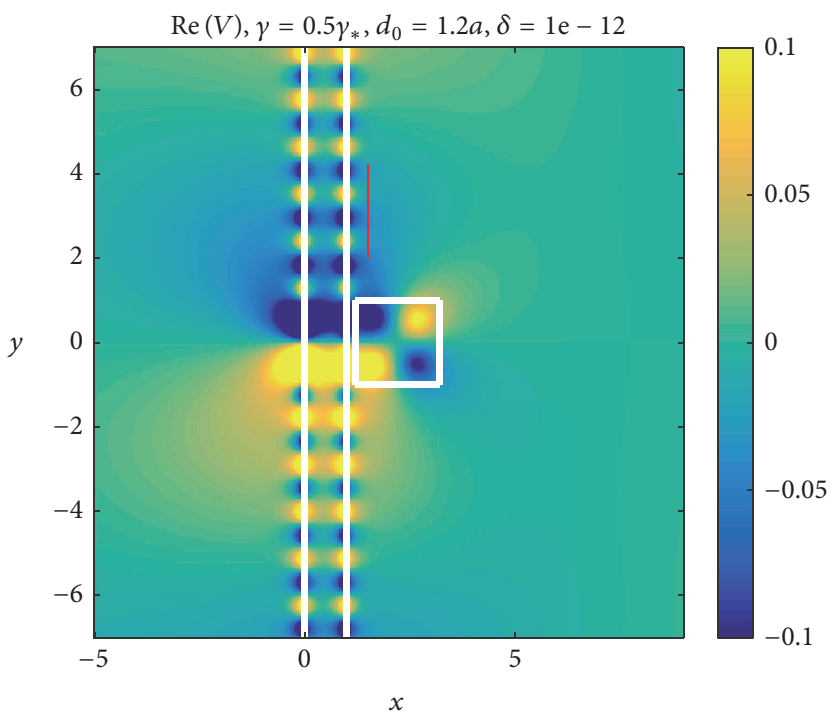

(c)

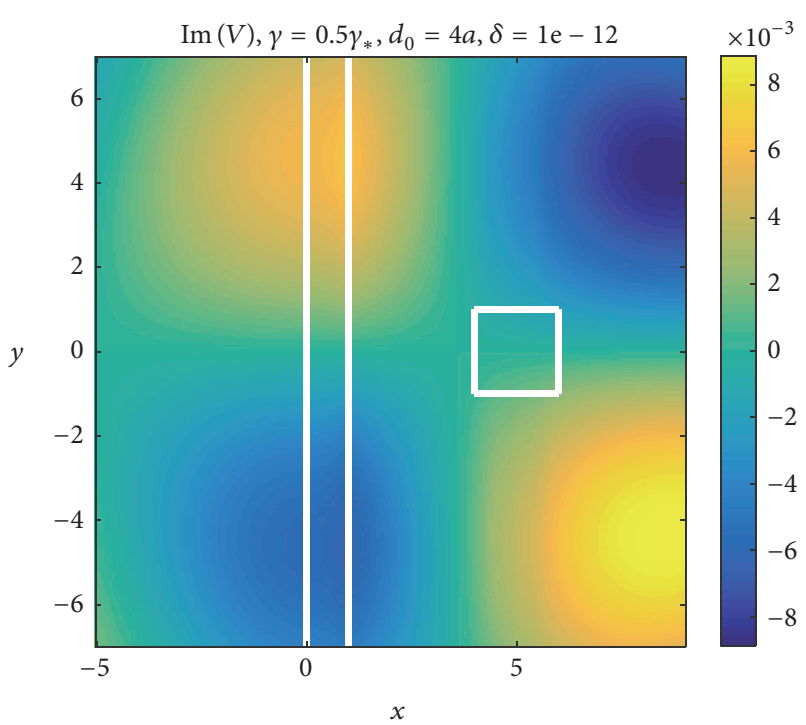

(b)

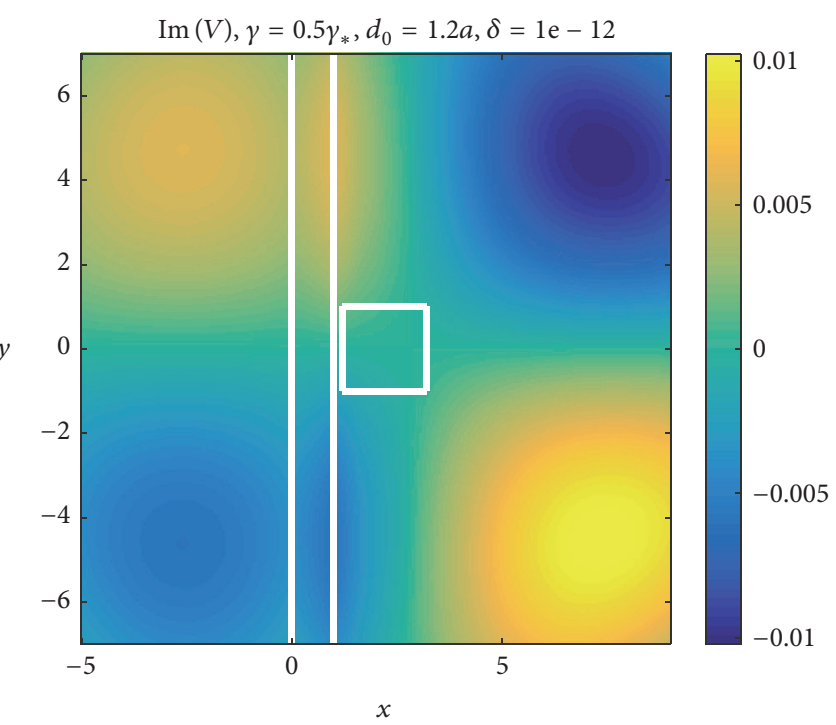

(d)

FIgURE 8: This is a plot of $V$, the solution to (4), when $f$ is the function in (49) and $\gamma=0.5 \gamma_{*}$ : (a) $\operatorname{Re}(V)$ and (b) $\operatorname{Im}(V)$ for $d_{0}=4 a$; (c) $\operatorname{Re}(V)$ and (d) $\operatorname{Im}(V)$ for $d_{0}=1.2 a$. To make the behavior of $\operatorname{Re}(V)$ clearer, in (a) and (c) we clipped the maximum and minimum values in each plot to 0.1 (yellow) and -0.1 (blue), respectively. The vertical red line in (c) extends a distance of $2 \lambda_{\gamma}$, where $\lambda_{\gamma}$ is defined in (66).

of $V$ is also independent of the source $f$. We emphasize that this is speculative at best, but it would be interesting to investigate further.

To illustrate how drastically different the behavior of $V$ is for $\gamma>\gamma_{*}$ and $\gamma<\gamma_{*}$, in Figure 9 we plotted $V$ corresponding to a dipole source located at $d_{0}=1.2 a$ for two different values of $\gamma$. In Figures 9(a) and 9(b), we took $\gamma=1.01 \gamma_{*}$ while in Figures 9(c) and 9(d) we took $\gamma=0.99 \gamma_{*}$. The ALR is present when $\gamma<\gamma_{*}$ in Figure 9(c); on the other hand, in Figure 9(a) there are a few oscillations near the $x$-axis, but they quickly die out as $|y|$ grows.

Unfortunately, we cannot provide a figure analogous to Figure 3 for $E_{\delta}(a)$ when $f$ is a dipole source-MATLAB is unable to accurately compute the integral

$$
\int_{1}^{\infty} L_{\delta}(p ; \gamma) \mathrm{d} p
$$

because $\left|g_{\delta}(p ; \gamma)\right|$ is very close to 0 near the roots of $g_{0}(p ; \delta)$ for small values of $\delta$ (see Conjecture 12). However, to get a sense of what is going on, we plotted $L_{\delta}(p ; \gamma)$ on a logarithmic scale for a dipole source $f$ with $\gamma=0.99 \gamma_{*}$ in Figures 10(a) and $10(\mathrm{~b})$ and $\gamma=1.01 \gamma_{*}$ in Figures 10(c) and 10(d). Each curve is $L_{\delta}(p ; \gamma)$ as a function of $p$ for various values of $\delta$. In Figures 10(a) and 10(b), where $\gamma<\gamma_{*}$, we see that $L_{\delta}(p ; \gamma)$ is quite large near the poles of $g_{0}(p ; \gamma)$, even if $\delta=10^{-4}$. Additionally, on comparing the $y$-axis scales in Figures 10(a) and 10(b), we note that the poles seem somewhat less severe in Figure 10(a) than in Figure 10(b), which, in combination with results from the quasistatic regime [24], lends credence 


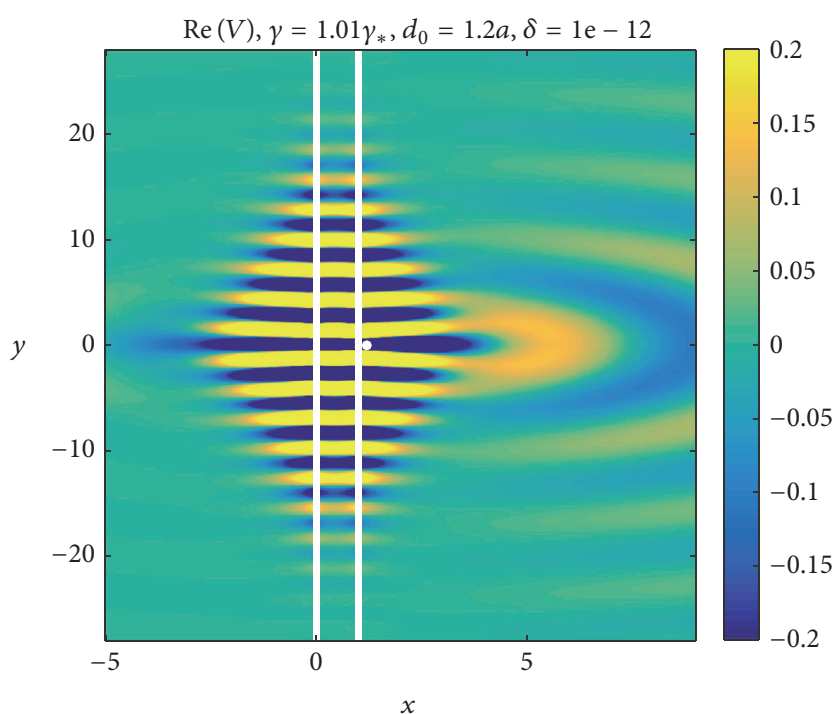

(a)

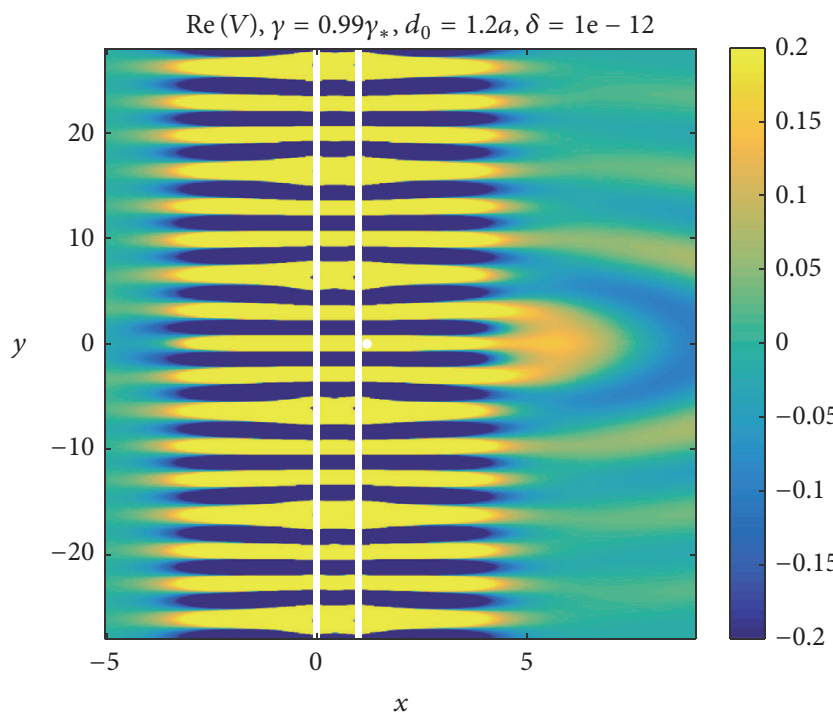

(c)

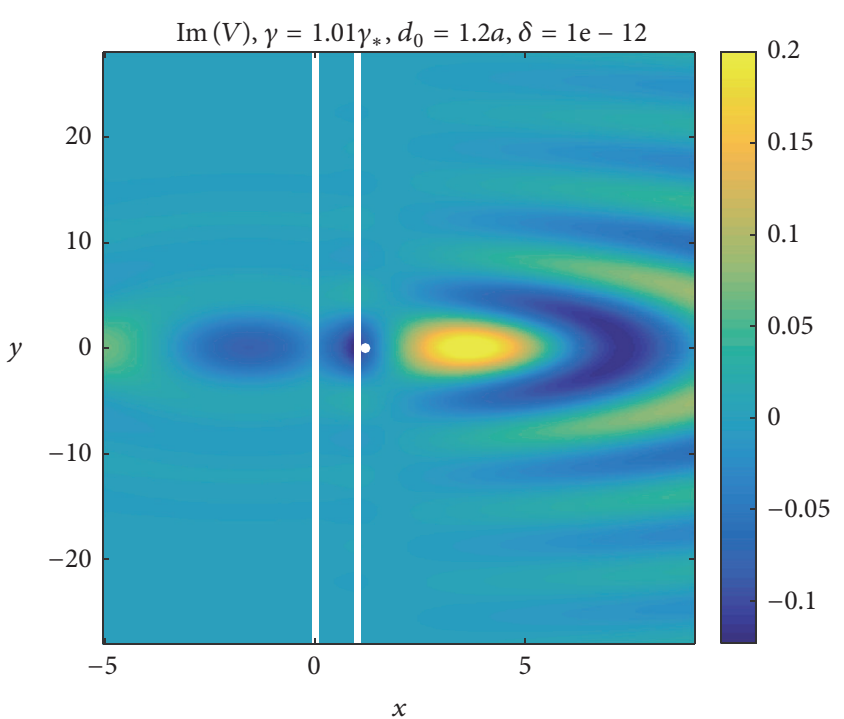

(b)

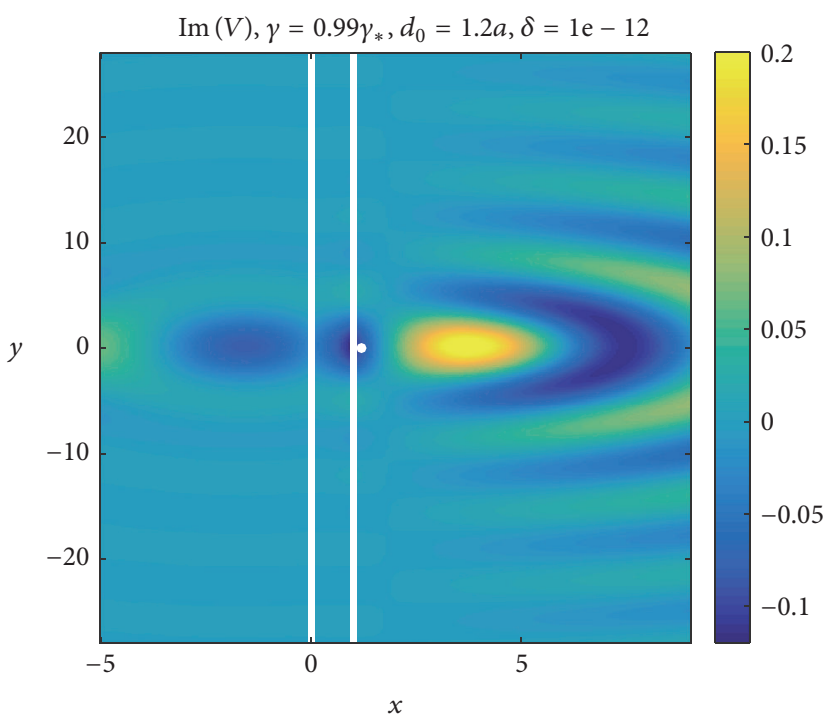

(d)

Figure 9: This is a plot of $V$, the solution to (4), when $f$ is a dipole located at $d_{0}=1.2 a$ : (a) $\operatorname{Re}(V)$ and (b) $\operatorname{Im}(V)$ for $\gamma=1.01 \gamma_{*}$; (c) $\operatorname{Re}(V)$ and (d) $\operatorname{Im}(V)$ for $\gamma=0.99 \gamma_{*}$. To make the behavior of $V$ clearer, in (a), (c), and (d) we clipped the maximum and minimum values in each plot to 0.1 (yellow) and -0.1 (blue), respectively.

to our conjecture (Conjecture 14) that ALR may be present only if the source is located close enough to the lens. On the other hand, in Figures 10(c) and 10(d), $\gamma>\gamma_{*}$ and we see that $L_{\delta}(p ; \gamma)$ remains bounded regardless of $d_{0}$ (in Figure 10, all of the functions rapidly tend to 0 for larger values of $p$ (not shown in the figure)).

4.2. Sources for Which ALR Does Not Occur. When $0<\gamma<$ $\gamma_{*}$, the conjectures from the previous section suggest that the zeros of $g_{0}(p ; \gamma)$ are responsible for forcing $E_{\delta}(a)$ to blow up in the limit as $\delta \rightarrow 0^{+}$. This begs the question of whether one can design a (realistic) source in the finite-frequency regime (with $0<\gamma<\gamma_{*}$ ) that effectively cancels the poles that show up in the limit $\delta \rightarrow 0^{+}$. In other words, we would like to design a source such that $\left|I_{p}\right|=0$ exactly at the zeros of $g_{0}(p ; \gamma)$; heuristically, in the limit as $\delta \rightarrow 0^{+}$, this would force the integrand in (30) to remain bounded at the zeros of $g_{0}(p ; \gamma)$ and annihilate the anomalous localized resonance that occurs in this limit. Recall from (24) that

$$
I_{p}=\int_{d_{0}}^{d_{1}} \widehat{f}\left(s, k_{0} p\right) \mathrm{e}^{-k_{0} v_{m} s} \mathrm{~d} s .
$$

Lemma 5 implies that $g_{0}(p ; \gamma)$ has two roots $1<p_{\gamma}^{1}<$ $p_{\gamma}^{2}$. Using this and (68), we see that an "ALR-busting" source $f(x, y)$ can be constructed by choosing $f$ such that $\widehat{f}\left(s, k_{0} p_{\gamma}^{1}\right)=\widehat{f}\left(s, k_{0} p_{\gamma}^{2}\right)=0$ for all $s \in\left[d_{0}, d_{1}\right]$ (which implies $\left.I_{p_{\gamma}^{1}}=I_{p_{\gamma}^{2}}=0\right)$. We do not want to just choose any $\widehat{f}$ satisfying this property; however, we restrict ourselves to those sources 


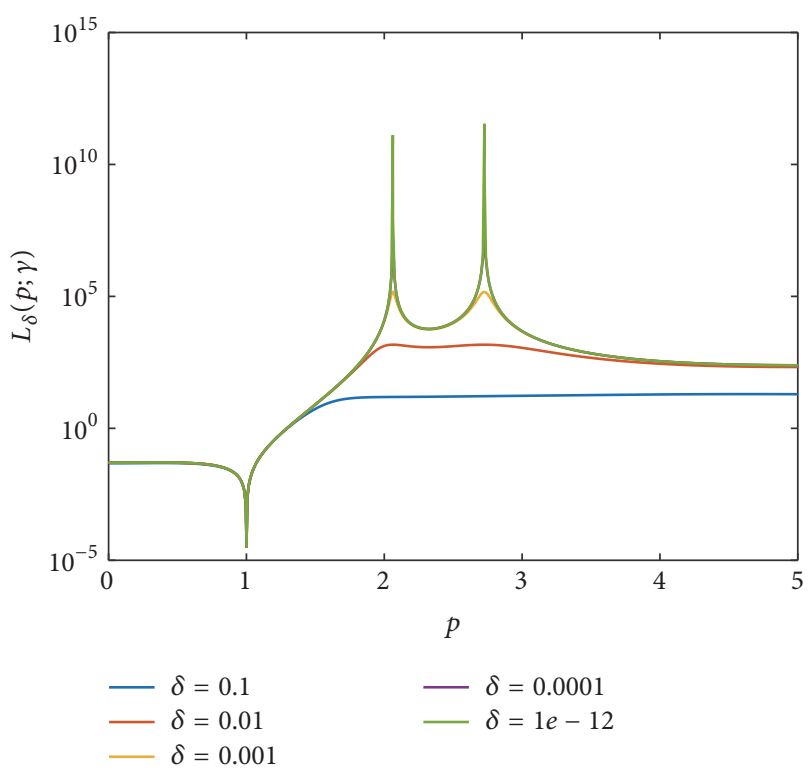

(a)

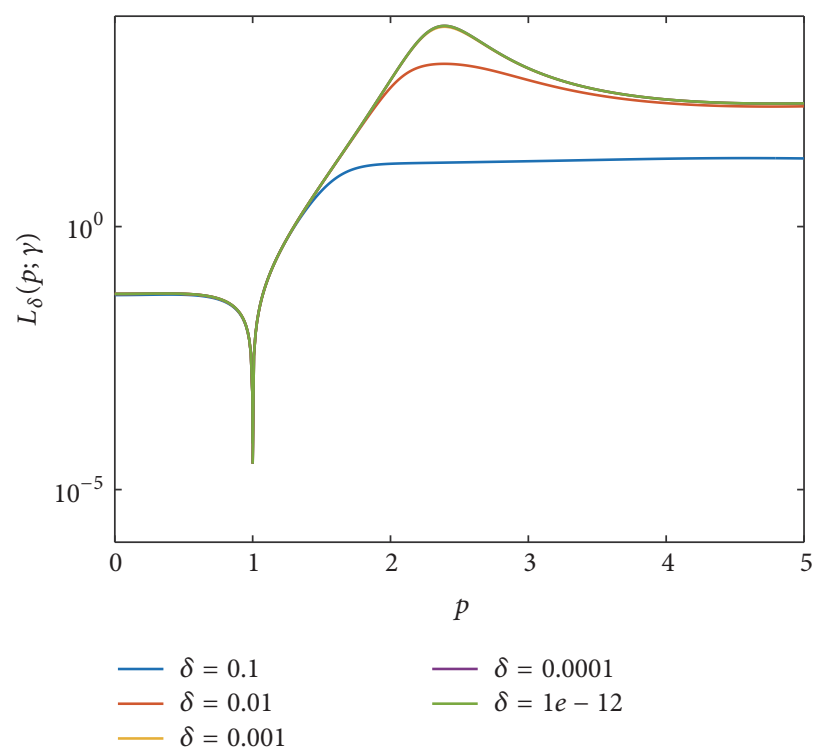

(c)

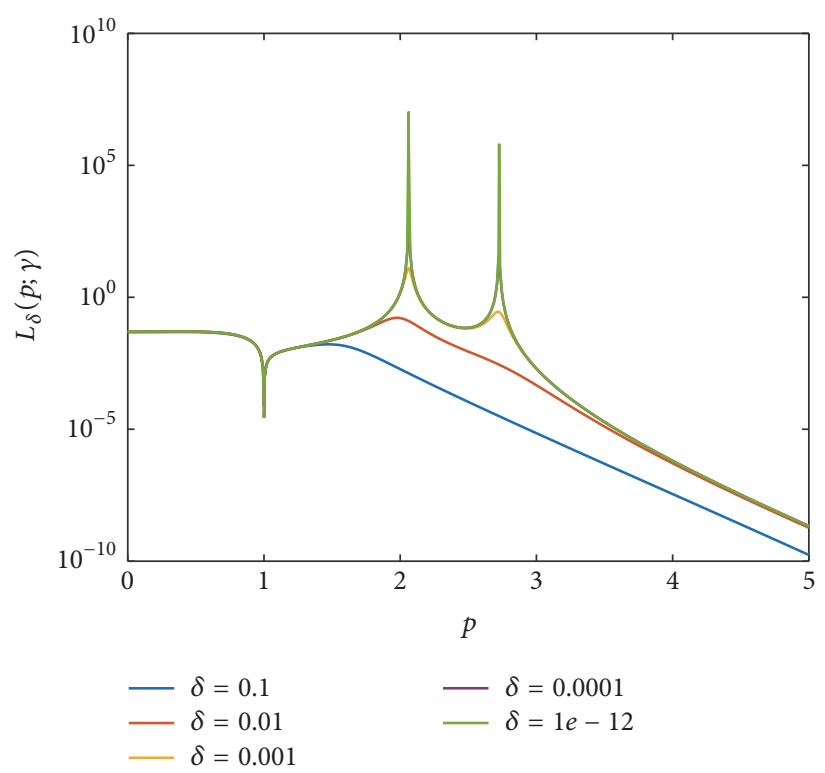

(b)

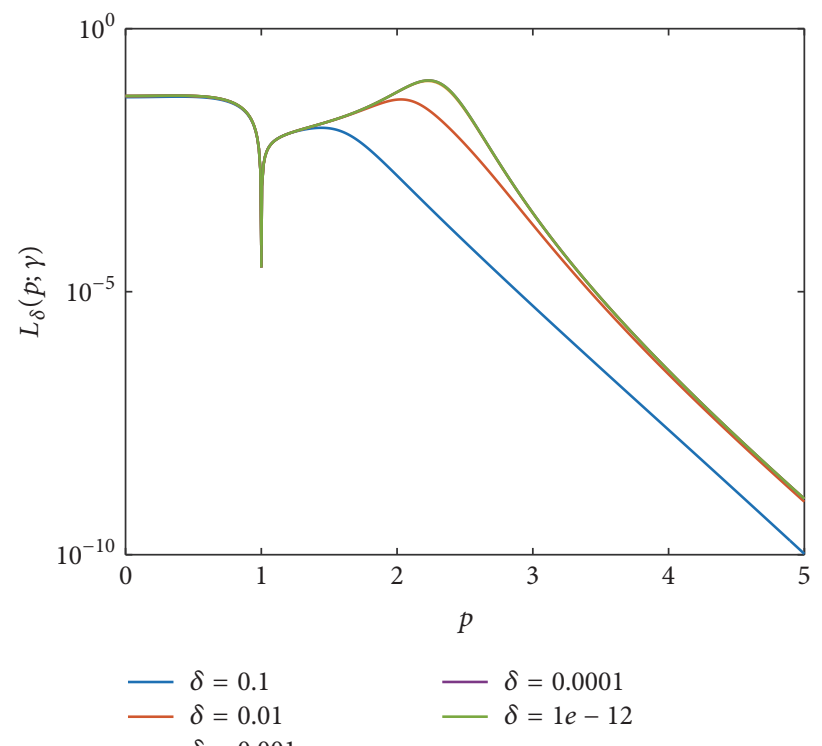

(d)

FIGURE 10: A plot of the integrand $L_{\delta}(p ; \gamma)$ from (36) for several parameter values. The separate curves in each plot represent different values of $\delta$, indicated in the legend: (a) $\gamma=0.99 \gamma_{*}$ and $d_{0}=1.2 a$; (b) $\gamma=0.99 \gamma_{*}$ and $d_{0}=4 a$; (c) $\gamma=1.01 \gamma_{*}$ and $d_{0}=1.2 a$; (d) $\gamma=1.01 \gamma_{*}$ and $d_{0}=4 a$.

$f \in L^{2}(\mathscr{M})$ with compact support. In summary, we make the following conjecture.

Conjecture 16. Suppose $f \in L^{2}(\mathscr{M})$ has compact support and

$$
\widehat{f}\left(x, k_{0} p_{\gamma}^{1}\right)=\widehat{f}\left(x, k_{0} p_{\gamma}^{2}\right)=0,
$$

where $1<p_{\gamma}^{1}<p_{\gamma}^{2}$ are the zeros of $g_{0}(p ; \gamma)$ from Lemma 5 and $k_{0} p_{\gamma}^{j}$ are zeros of order at least 1 for $\hat{f}\left(x, k_{0} p\right)$. Then there is $\delta_{\gamma}>0$ and a constant $C_{\gamma}>0$ such that $E_{\delta}(a) \leq C_{\gamma}$ for all $0<\delta \leq \delta_{\gamma}$.
There are many sources that satisfy the hypotheses of this theorem. We will present 2 examples here. First, consider

$$
\widehat{f}(x, q)=-\mathrm{i} \chi_{\left(d_{0}, d_{1}\right)}(x) \operatorname{sinc}\left(\alpha_{1} q\right) \sin \left(\alpha_{2} q\right),
$$

where $\operatorname{sinc}(x)=\sin (x) / x$,

$$
\chi_{\left(d_{0}, d_{1}\right)}(x)= \begin{cases}1 & \text { for } d_{0}<x<d_{1}, \\ 0 & \text { otherwise }\end{cases}
$$




$$
\begin{aligned}
& \alpha_{1} \equiv \frac{\pi}{k_{0} p_{\gamma}^{1}}, \\
& \alpha_{2} \equiv \frac{\pi}{k_{0} p_{\gamma}^{2}} .
\end{aligned}
$$

Then $\hat{f}(x, \cdot) \in L^{2}(\mathbb{R})$ and, hence, $f(x, \cdot) \in L^{2}(\mathbb{R})$ by the Plancherel theorem; moreover, $\widehat{f}\left(x, k_{0} p_{\gamma}^{1}\right)=\widehat{f}\left(x, k_{0} p_{\gamma}^{2}\right)=0$, where the zeros are order 1 . Finally, by direct calculations we have

$$
\begin{aligned}
& f(x, y)=\chi_{\left(d_{0}, d_{1}\right)}(x) \cdot \frac{1}{4 \alpha_{1}}\left[H\left(-y-\alpha_{1}-\alpha_{2}\right)\right. \\
& -H\left(-y-\alpha_{1}+\alpha_{2}\right)+H\left(-y+\alpha_{1}+\alpha_{2}\right) \\
& \left.-H\left(-y-\alpha_{2}+\alpha_{1}\right)\right],
\end{aligned}
$$

where $H(z)$ is the Heaviside step function; this $f \in L^{2}(\mathscr{M})$ has compact support and thus satisfies the hypotheses of Conjecture 16. We may also take

$$
\widehat{f}(x, q)=\chi_{\left(d_{0}, d_{1}\right)}(x) J_{0}\left(\beta_{0} q\right) J_{1}\left(\beta_{1} q\right),
$$

where $J_{0}$ and $J_{1}$ are the Bessel functions of the first kind of orders 0 and 1 , respectively, and $\beta_{0}$ and $\beta_{1}$ are such that $J_{0}\left(\beta_{0} k_{0} p_{\gamma}^{1}\right)=J_{1}\left(\beta_{1} k_{0} p_{\gamma}^{2}\right)=0$ (we note that these zeros are also of order 1). Because the Bessel functions of the first kind are $O\left(q^{-1 / 2}\right)$ as $q \rightarrow \infty[25]$, we have $\widehat{f}(x, \cdot) \in L^{2}(\mathbb{R})$. By the convolution theorem for Fourier transforms,

$$
f(x, y)=\chi_{\left(d_{0}, d_{1}\right)}(x)\left(f_{0} * f_{1}\right)(y),
$$

where $*$ denotes convolution and $f_{0}$ and $f_{1}$ are the inverse Fourier transforms of $J_{0}\left(\beta_{0} q\right)$ and $J_{1}\left(\beta_{1} q\right)$, respectively; in particular, we obtain

$$
\begin{aligned}
& f_{0}(y)=\frac{1}{\pi \sqrt{\beta_{0}^{2}-y^{2}}} \chi_{\left(-\beta_{0}, \beta_{0}\right)}(y), \\
& f_{1}(y)=\frac{-y}{\beta_{1} \pi \sqrt{\beta_{1}^{2}-y^{2}}} \chi_{\left(-\beta_{1}, \beta_{1}\right)}(y) .
\end{aligned}
$$

Although the convolution in (74) is difficult to compute analytically, since $f_{0}$ and $f_{1}$ both have compact support, the convolution of $f_{0}$ with $f_{1}$ will as well. Thus $f$ as defined in (74) is in $L^{2}(\mathscr{M})$ and has compact support.

In Figures 11(a) and 11(b) we plot $\operatorname{Re}(V)$ and $\operatorname{Im}(V)$, respectively, for the source from (70) (equivalently, (72)); in Figures $11(\mathrm{c})$ and $11(\mathrm{~d})$, we plot $\operatorname{Re}(V)$ and $\operatorname{Im}(V)$, respectively, for the source from (73) (equivalently, (74)). We take the same parameters that we used in Figures 7(c), 7(d), 8(c), and 8(d), namely, $d_{0}=1.2 a$ and $\gamma=0.5 \gamma_{*}$. In stark contrast with those figures, the solution $V$ is well-behaved in Figure 11.

4.2.1. Current Sources for Which ALR Does Not Occur. In the monochromatic electromagnetic setting, $f$ must satisfy additional restrictions for it to represent a realistic (divergencefree) current source-see Section A.5. In particular, the function $f$ should be in $L^{2}(\mathscr{M})$ with compact support and be of the form

$$
f=\mu_{0}\left(\frac{\partial \widetilde{J}_{y}}{\partial x}-\frac{\partial \widetilde{J}_{x}}{\partial y}\right)
$$

for a current

$$
\widetilde{\mathbf{J}}=\widetilde{J}_{x}(x, y) \mathbf{e}_{x}+\widetilde{J}_{y}(x, y) \mathbf{e}_{y}
$$

satisfying the continuity equation

$$
\frac{\partial \widetilde{J}_{x}}{\partial x}+\frac{\partial \widetilde{J}_{y}}{\partial y}=0 .
$$

We now construct a source $f$ satisfying the hypotheses of Conjecture 16 that is of the form (76)-(78). For simplicity, we assume that the current from (77) has the form

$$
\begin{aligned}
& \tilde{J}_{x}(x, y)=r_{1}(x) t_{1}(y), \\
& \tilde{J}_{y}(x, y)=r_{2}(x) t_{2}(y) .
\end{aligned}
$$

with $r_{1}, r_{2}, t_{1}, t_{2}$ smooth enough. Then continuity equation (78) becomes

$$
r_{1}^{\prime}(x) t_{1}(y)+r_{2}(x) t_{2}^{\prime}(y)=0 .
$$

Taking the Fourier transform of this equation with respect to $y$ gives

$$
r_{1}^{\prime}(x) \widehat{t}_{1}(q)+r_{2}(x) \mathrm{i} q \widehat{t}_{2}(q)=0 .
$$

We further simplify the problem by taking

$$
\widehat{t}_{1}(q)=\mathrm{i} q \widehat{t}_{2}(q) \text {. }
$$

Then (81) becomes

$$
\left[r_{1}^{\prime}(x)+r_{2}(x)\right] \widehat{t}_{1}(q)=0,
$$

which is satisfied for all $x$ and $q$ if

$$
r_{2}(x)=-r_{1}^{\prime}(x) .
$$

Then (76), (79), (82), and (84) imply that

$$
\begin{aligned}
\widehat{f}(x, q) & =\mu_{0}\left[r_{2}^{\prime}(x) \widehat{t}_{2}(q)-r_{1}(x) \mathrm{i} q t_{1}(q)\right] \\
& =\mu_{0}\left[-r_{1}^{\prime \prime}(x) \widehat{t}_{2}(q)+r_{1}(x) q^{2} \widehat{t}_{2}(q)\right] .
\end{aligned}
$$

At this point, $f$ satisfies (76)-(78).

By the Plancherel theorem and (85), $f \in L^{2}(\mathscr{M})$ if and only if $\widehat{t}_{2}(q) \in L^{2}(\mathbb{R})$ and $q^{2} \widehat{t}_{2}(q) \in L^{2}(\mathbb{R})$. We must also be careful to also choose $\widehat{t}_{2}(q)$ in such a way that $\widehat{t}_{2}\left(k_{0} p_{\gamma}^{1}\right)=$ $\widehat{t}_{2}\left(k_{0} p_{\gamma}^{2}\right)=0$ and $f(x, y)=\mu_{0}\left[-r_{1}^{\prime \prime}(x) t_{2}(y)-r_{1}(x) t_{2}^{\prime \prime}(y)\right]$ has compact support in $y$.

There are many examples of functions that accomplish these tasks. Unfortunately, the functions in (72) and (74) lead to current sources that are discontinuous and, hence, not divergence-free, so we need to be a bit more careful. To 


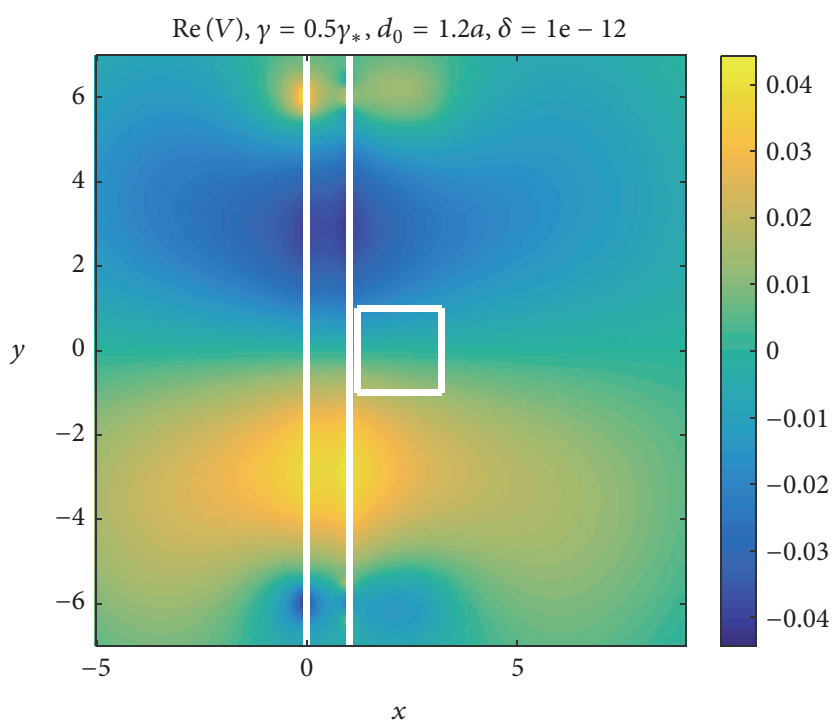

(a)

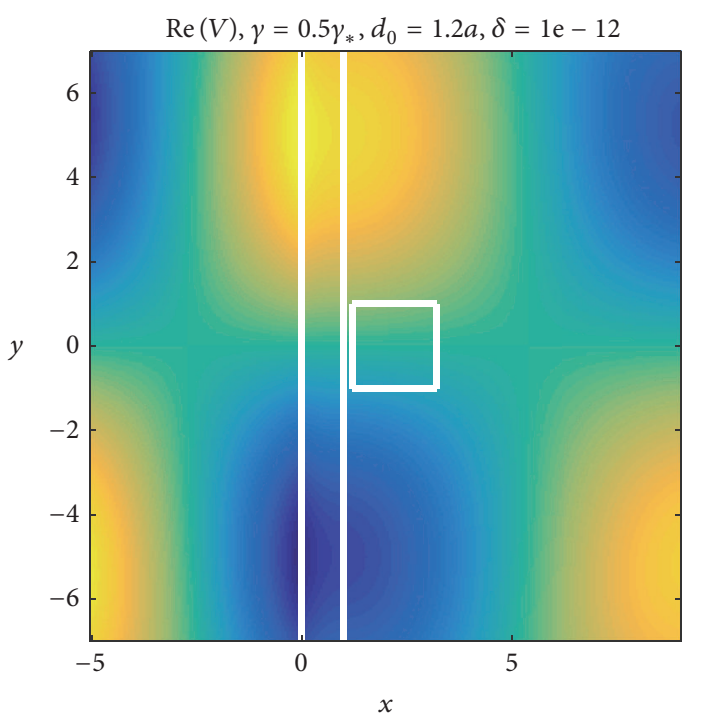

(c)

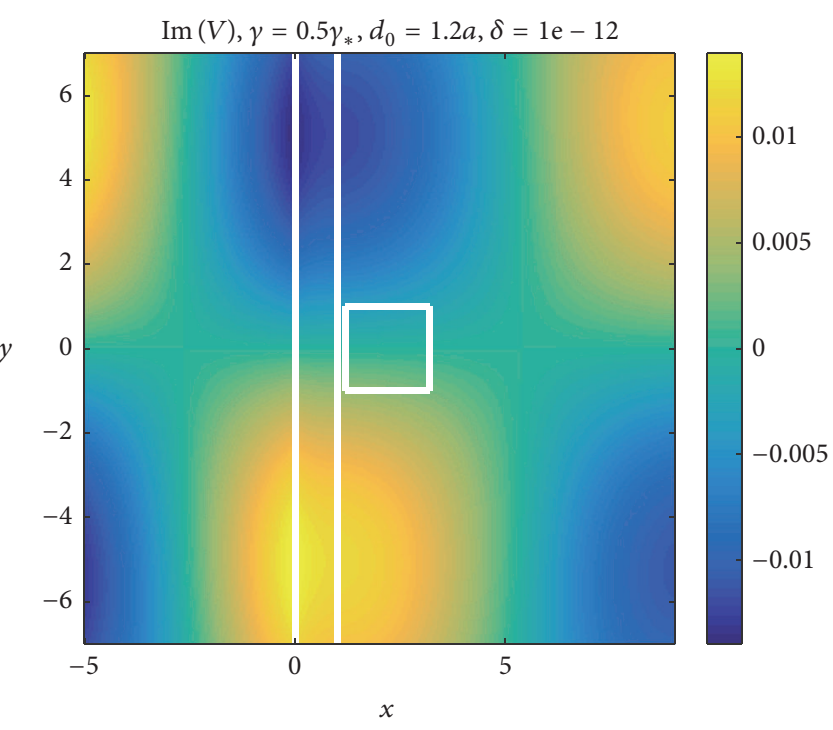

(b)

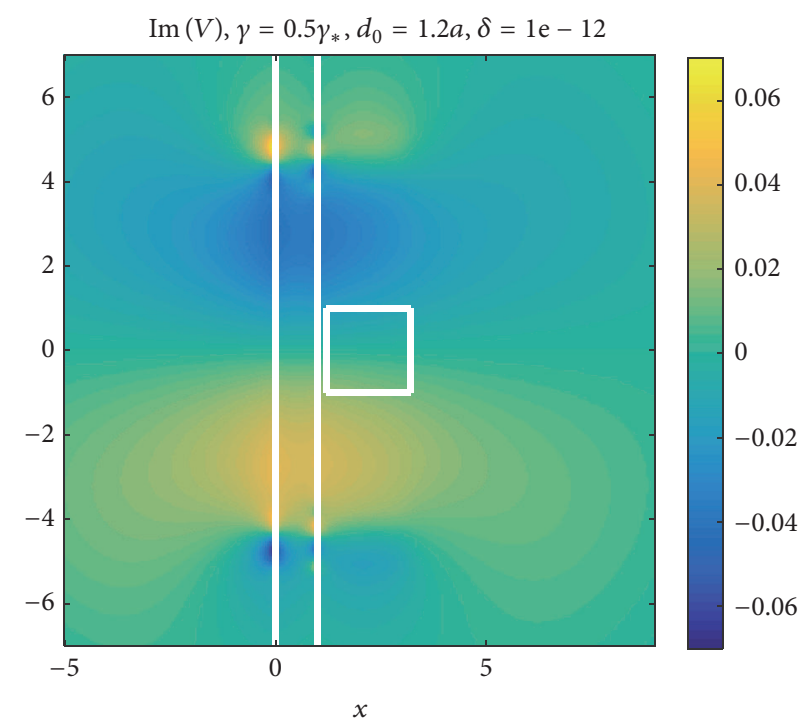

(d)

Figure 11: This is a plot of $V$, the solution to (4), for two different sources with $d_{0}=1.2 a$ and $\gamma=0.5 \gamma_{*}$ : (a) $\operatorname{Re}(V)$ and (b) $\operatorname{Im}(V)$ for $f$ as in (70) (equivalently, (72)); (c) $\operatorname{Re}(V)$ and $(\mathrm{d}) \operatorname{Im}(V)$ for $f$ as in (73) (equivalently (74)).

find a smooth current with compact support satisfying our requirements, we take

$$
\begin{aligned}
& \widehat{t}_{2}(q)=\operatorname{sinc}^{3}\left(\alpha_{1} q\right) \operatorname{sinc}^{2}\left(\alpha_{2} q\right) \\
& \alpha_{1} \equiv \frac{\pi}{k_{0} p_{\gamma}^{1}}, \alpha_{2} \equiv \frac{\pi}{k_{0} p_{\gamma}^{2}} .
\end{aligned}
$$

Then $\widehat{t}_{2}(q) \in L^{2}(\mathbb{R}), q^{2} \widehat{t}_{2}(q) \in L^{2}(\mathbb{R})$, and $\widehat{f}\left(x, k_{0} p_{\gamma}^{1}\right)=$ $\hat{f}\left(x, k_{0} p_{\gamma}^{2}\right)=0$ (here the zeros are of orders 3 and 2 , resp., so they are stronger than what we need according to Conjecture 16; we take these higher-order zeros to ensure that $t_{2}(y)$ and $t_{2}^{\prime \prime}(y)$ are both continuous; there may be other choices of continuous functions $t_{2}(y)$ and $t_{2}^{\prime \prime}(y)$ such that $\widehat{t}_{2}(q) \in L^{2}(\mathbb{R})$ has zeros of order 1 at $q=k_{0} p_{1}$ and $\left.q=k_{0} p_{2}\right)$. One possible choice of $r_{1}(x)$ from (85) is

$$
r_{1}(x)= \begin{cases}C\left[\frac{2}{d_{1}-d_{0}}\left(x-d_{0}\right)-1\right]^{3}\left[\left|\frac{2}{d_{1}-d_{0}}\left(x-d_{0}\right)-1\right|-1\right]^{3} & \text { for } d_{0} \leq x \leq d_{1} \\ 0 & \text { otherwise }\end{cases}
$$




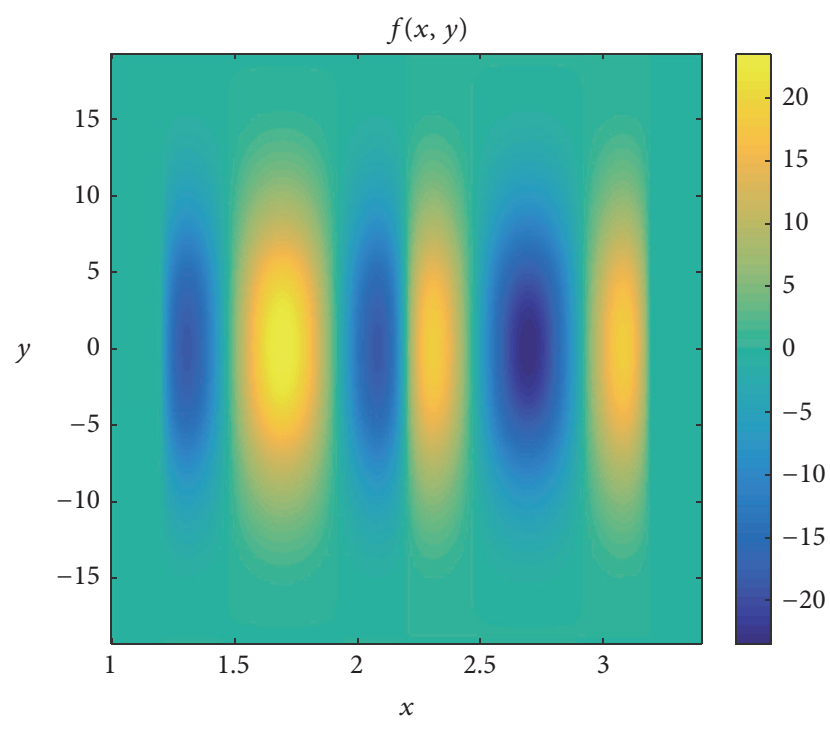

Figure 12: This is the source $f$ defined by (85)-(87) with the parameters $d_{0}=1.2 a$ and $d_{1}=d_{0}+2$.

where $C$ is a nonzero constant. The function $r_{1}(x)$ is twice continuously differentiable and has compact support, so $r_{1}^{\prime \prime}(x)$ is continuous with compact support.

Finally, $\hat{f}(x, q)$ may be computed via (85), (86), and (87). We note that the inverse Fourier transform of $\hat{f}(x, q)$ can be computed analytically; for the benefit of the reader, we avoid writing out the expression. Importantly, $f$ is continuous with compact support.

The current source corresponding to this $f$ may be computed via (77), (79), (82), (84), (86), and (87). We emphasize that both $\widetilde{J}_{x}(x, y)$ and $\widetilde{J}_{y}(x, y)$ are continuously differentiable functions with compact support in $\mathscr{M}$.

In Figure 12, we plot the source $f$ defined by (85)-(87) with $C=10^{3} \mu_{0}^{-1}$. In Figures 13(a) and 13(b), we plot $\operatorname{Re}(V)$ and $\operatorname{Im}(V)$, respectively, corresponding to this source. As expected, we see that there is no resonant region near the slab even though the source is quite close to the slab $\left(d_{0}=1.2 a\right)$, $\gamma=0.5 \gamma_{*}$, and $\delta=10^{-12}$.

\section{Appendix}

\section{A. Proofs and Derivations Omitted in the Text}

In this appendix, we provide detailed proofs we omitted in the main body of the paper.

A.1. Proof of Lemma 5. Setting $g_{0}(p ; \gamma)=0$, defining a new variable $s \equiv p^{2}$, and simplifying, we find that $g_{0}(p ; \gamma)=0$ is equivalent to having

$$
s+\sqrt{s^{2}-1}=\mathrm{e}^{\gamma \sqrt{s+1}} .
$$

We define

$$
G_{0}(s ; \gamma) \equiv s+\sqrt{s^{2}-1}-\mathrm{e}^{\gamma \sqrt{s+1}} \quad \text { for } s \geq 0, \gamma>0 .
$$

Then $g_{0}(p ; \gamma)=0$ if and only if $G_{0}(s ; \gamma)=0$. We will complete the proof of the lemma in several steps.

(1) Claim. $G_{0}(s ; \gamma) \neq 0$ for $s \leq 1$.

Proof of Claim. For $s \leq 1$,

$$
G_{0}(s ; \gamma)=s-\mathrm{e}^{\gamma \sqrt{s+1}}+\mathrm{i} \sqrt{1-s^{2}} .
$$

Since $\operatorname{Im} G_{0}(s ; \gamma)=\sqrt{1-s^{2}}>0$ for $s<1$, the only point at which $G_{0}(s ; \gamma)$ could possibly be 0 is $s=1$. But, for $\gamma>0$,

$$
G_{0}(1 ; \gamma)=1-\mathrm{e}^{\sqrt{2} \gamma}<0 .
$$

In particular, this proves that if $p_{0} \geq 0$ is a root of $g_{0}(p ; \gamma)$, then $p_{0}>1$.

(2) Claim. For $\gamma \geq \sqrt{\mathrm{e}} /(\mathrm{e}+1) \approx 0.4434$, the function $s \mapsto G_{0}(s ; \gamma)$ is concave for $s>1$.

Proof of Claim. For $s>1$, we have

$$
\begin{aligned}
G_{0}(s ; \gamma)= & s+\sqrt{s^{2}-1}-\mathrm{e}^{\gamma \sqrt{s+1}}, \\
\frac{\partial^{2} G_{0}}{\partial s^{2}}= & -\frac{1}{\left(s^{2}-1\right)^{3 / 2}} \\
& +\frac{\gamma \mathrm{e}^{\gamma \sqrt{s+1}}}{4(s+1)^{3 / 2}}(1-\gamma \sqrt{s+1}) .
\end{aligned}
$$

We note that $1-\gamma \sqrt{s+1}<0$ for $\gamma>1 / \sqrt{2} \approx 0.7071$ and $s>1$. This proves the claim for $\gamma>1 / \sqrt{2}$.

Now consider

$$
\frac{\sqrt{\mathrm{e}}}{\mathrm{e}+1} \leq \gamma \leq \frac{1}{\sqrt{2}} .
$$

We note that $1-\gamma \sqrt{s+1}<0$ for $s>\gamma^{-2}-1$, so $\partial^{2} G_{0} / \partial s^{2}<0$ for $s>\gamma^{-2}-1$. Then, for $1<s \leq \gamma^{-2}-1$, we have

$$
\frac{\partial^{2} G_{0}}{\partial s^{2}}<0 \Longleftrightarrow
$$

$\gamma(s-1)^{3 / 2} \mathrm{e}^{\gamma \sqrt{s+1}}(1-\gamma \sqrt{s+1})<4$.

For $1<s \leq \gamma^{-2}-1$, the left-hand side of the above inequality satisfies

$$
\begin{gathered}
\gamma(s-1)^{3 / 2} \mathrm{e}^{\gamma \sqrt{s+1}}(1-\gamma \sqrt{s+1}) \\
\leq \mathrm{e} \gamma\left(\gamma^{-2}-2\right)^{3 / 2}(1-\sqrt{2} \gamma) .
\end{gathered}
$$

Using calculus and Maple, it can be shown that

$$
\begin{gathered}
\max _{\gamma \in[\sqrt{\mathrm{e}} /(\mathrm{e}+1), 1 / \sqrt{2}]} \mathrm{e} \gamma\left(\gamma^{-2}-2\right)^{3 / 2}(1-\sqrt{2} \gamma) \\
=\frac{\left(\mathrm{e}^{2}+1\right)^{3 / 2}(\mathrm{e}-\sqrt{2 \mathrm{e}}+1)}{(\mathrm{e}+1)^{2}} \approx 2.4370 .
\end{gathered}
$$




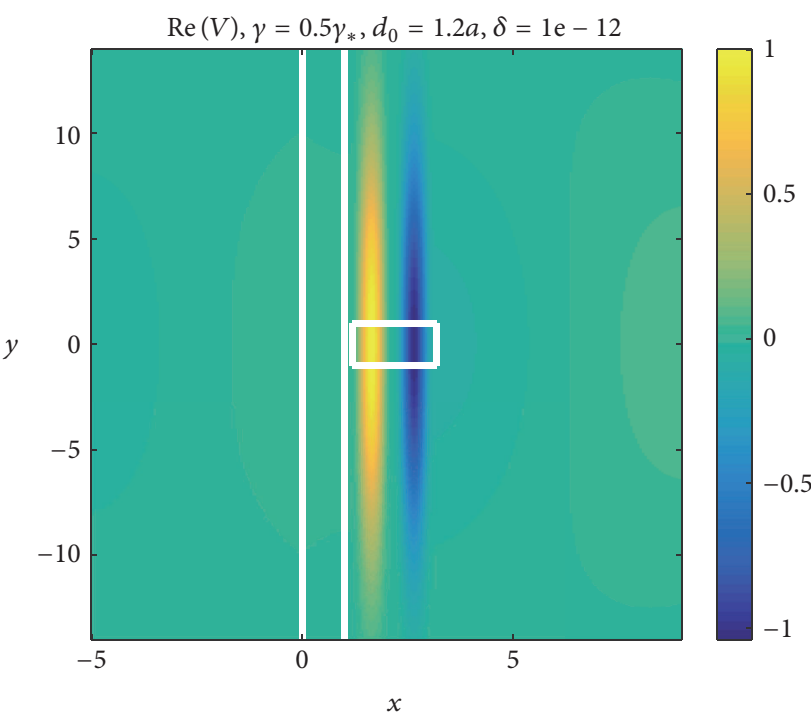

(a)

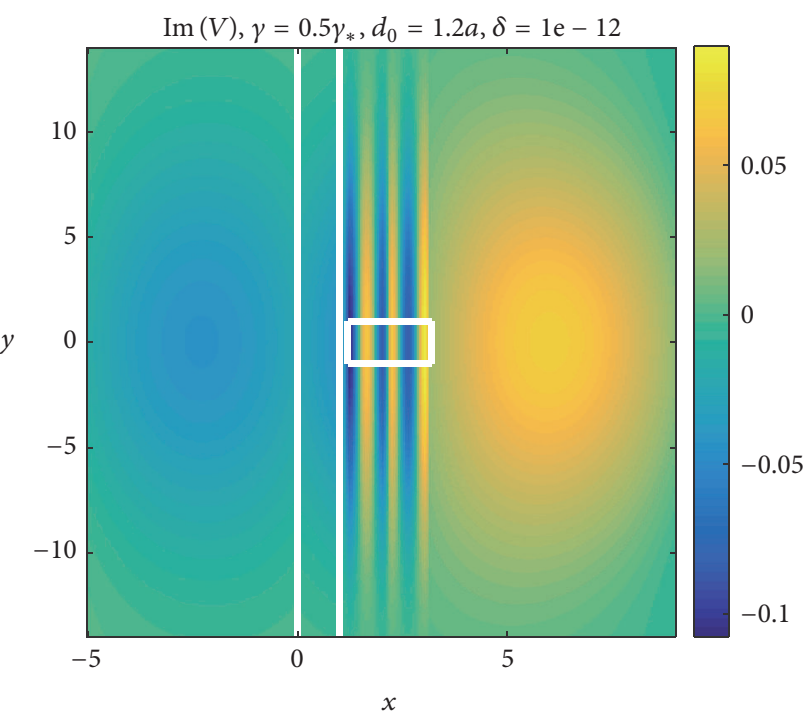

(b)

FIgURE 13: In this figure, we plot (a) $\operatorname{Re}(V)$ and (b) $\operatorname{Im}(V)$, where $V$ is the solution to (4) corresponding to the source $f$ defined through (85)(87) with the parameters $d_{0}=1.2 a, \gamma=0.5 \gamma_{*}$, and $\delta=10^{-12}$. To make the behavior of $V$ clearer, we clipped the maximum and minimum values in each plot to 0.2 (yellow) and -0.2 (blue), respectively.

In combination with (A.7) and (A.8), this implies

$$
\frac{\partial^{2} G_{0}}{\partial s^{2}}<0
$$

for $s>1$ as long as $\gamma \geq \sqrt{\mathrm{e}} /(\mathrm{e}+1)$ and verifies the claim.

(3) Claim. For $0<\gamma<\sqrt{\mathrm{e}} /(\mathrm{e}+1) \approx 0.4434$, the function $s \mapsto G_{0}(s ; \gamma)$ has two real zeros $1<s_{\gamma}^{1}<s_{\gamma}^{2}$.

Proof of Claim. We begin by defining the functions

$$
\begin{aligned}
G_{1}(s) & \equiv s+\sqrt{s^{2}-1}, \\
G_{2}(s ; \gamma) & \equiv \mathrm{e}^{\gamma \sqrt{s+1}}
\end{aligned}
$$

for $s>1$ and $\gamma \in(0, \sqrt{\mathrm{e}} /(\mathrm{e}+1))$. Then

$$
G_{0}(s ; \gamma)=G_{1}(s)-G_{2}(s ; \gamma) \text {. }
$$

For $s>1$ we have

$$
\begin{aligned}
\frac{\mathrm{d} G_{1}}{\mathrm{~d} s} & =1+\frac{s}{\sqrt{s^{2}-1}}>2, \\
\frac{\mathrm{d}^{2} G_{1}}{\mathrm{~d} s^{2}} & =-\frac{1}{\left(s^{2}-1\right)^{3 / 2}}<0 .
\end{aligned}
$$

Similarly, for $s>1$ we have

$$
\begin{aligned}
\frac{\partial G_{2}}{\partial s} & =\frac{\gamma}{2} \cdot \frac{\mathrm{e}^{\gamma \sqrt{s+1}}}{\sqrt{s+1}}>0, \\
\frac{\partial^{2} G_{2}}{\partial s^{2}} & =\frac{\gamma \mathrm{e}^{\gamma \sqrt{s+1}}}{4(s+1)^{3 / 2}}(\gamma \sqrt{s+1}-1) .
\end{aligned}
$$

Then $\partial^{2} G_{2} / \partial s^{2}<0$ for $s<\gamma^{-2}-1$ and $\partial^{2} G_{2} / \partial s^{2}>0$ for $s>\gamma^{-2}-1$.

Next, we note that

$$
G_{0}(1 ; \gamma)=G_{1}(1 ; \gamma)-G_{2}(1 ; \gamma)=1-\mathrm{e}^{\sqrt{2} \gamma}<0
$$

In addition, we have

$$
\frac{\partial G_{2}}{\partial s}(1 ; \gamma) \leq \frac{\partial G_{2}}{\partial s}\left(1 ; \frac{\sqrt{\mathrm{e}}}{\mathrm{e}+1}\right) \approx 0.2935<2 .
$$

Because $\partial G_{2} / \partial s$ is decreasing for $1<s<\gamma^{-2}-1$ and $\mathrm{d} G_{1} / \mathrm{d} s>2$ for $s>1$, the above inequality implies

$$
\frac{\partial G_{0}}{\partial s}=\frac{\mathrm{d} G_{1}}{\mathrm{~d} s}-\frac{\partial G_{2}}{\partial s}>0 \text { for } 1<s<\gamma^{-2}-1 \text {. }
$$

In fact, $G_{0}$ increases enough on this interval to become positive; we have

$$
\begin{aligned}
G_{0}\left(\gamma^{-2}-1 ; \gamma\right) & =\gamma^{-2}\left[1-(\mathrm{e}+1) \gamma^{2}+\sqrt{1-2 \gamma^{2}}\right] \\
& \geq \gamma^{-2}\left[1-\frac{\mathrm{e}}{\mathrm{e}+1}+\sqrt{1-\frac{2 \mathrm{e}}{(\mathrm{e}+1)^{2}}}\right] \\
& \approx 1.0479 \gamma^{-2}>0
\end{aligned}
$$

For $s>\gamma^{-2}-1, \partial G_{2} / \partial s$ increases without bound while $\mathrm{d}_{1} / \mathrm{d} s$ will get arbitrarily close to 2 ; the upshot of this is that $\partial G_{0} / \partial s$ becomes arbitrarily negative for $s$ large enough. 
In summary, we have $G_{0}(1 ; \gamma)<0$; the function $G_{0}(s ; \gamma)$ increases at least until $s=\gamma^{-2}-1$, where $G_{0}\left(\gamma^{-2}-1 ; \gamma\right)>0$. Next, $G_{0}(s ; \gamma)$ will continue increasing until $\partial G_{2} / \partial s$ becomes larger than $\mathrm{d} G_{1} / \mathrm{d} s$; then $G_{0}(s ; \gamma)$ will decrease toward $-\infty$ as $s$ approaches $\infty$. Thus $G_{0}(s ; \gamma)$ has 2 real zeros for $\gamma<\sqrt{\mathrm{e}} /(\mathrm{e}+1)$; by item (1) above, both of these zeros must be larger than 1 . Finally, since $G_{0}\left(\gamma^{-2}-1 ; \gamma\right)$ is strictly greater than 0 , by continuity the roots cannot be equal. This also proves that both of the zeros are of order 1 . This proves the claim.

We have shown that $G_{0}(s ; \gamma)$ has two real roots $1<s_{\gamma}^{1}<s_{\gamma}^{2}$ provided $0<\gamma<\sqrt{\mathrm{e}} /(\mathrm{e}+1)$. For $\gamma \geq \sqrt{\mathrm{e}} /(\mathrm{e}+1)$, the function $s \mapsto G_{0}(s ; \gamma)$ is concave for $s>1$ by item (2) above. Thus $G_{0}(s ; \gamma)$ has a unique maximum and will have two real roots of order 1 if the maximum is positive and no real roots if the maximum is negative.

Because the maximum of $G_{0}(s ; \gamma)$ is positive for $\gamma=$ $\sqrt{e} /(e+1)$ (see the proof of item (3) above) and $G_{0}(s ; \gamma)$ decreases at an exponential rate as a function of $\gamma$, there exists $\gamma_{*}>\sqrt{\mathrm{e}} /(\mathrm{e}+1)$ such that

$$
\max _{s>1} G_{0}(s ; \gamma) \begin{cases}>0 & \text { for } \gamma<\gamma_{*}, \\ <0 & \text { for } \gamma>\gamma_{*} .\end{cases}
$$

In particular, we have

$$
\gamma_{*}=\underset{\gamma>0}{\arg \min }\left[\max _{s>1} G_{0}(s ; \gamma)\right] .
$$

MATLAB gives $\gamma_{*} \approx 0.9373$. This completes the proof.

A.2. $I_{p}$ for Dipole Sources. In this section, we derive an explicit formula for $I_{q}$, defined in (24), when the source $f$ is a dipole. In particular, we consider a source of the form

$$
\begin{aligned}
f(x, y)= & \mathbf{d} \cdot \nabla\left[\delta\left(x-x_{0}\right) \delta\left(y-y_{0}\right)\right] \\
= & d_{x}\left[\frac{\partial}{\partial x} \delta\left(x-x_{0}\right)\right] \delta\left(y-y_{0}\right) \\
& +d_{y} \delta\left(x-x_{0}\right) \frac{\partial}{\partial y} \delta\left(y-y_{0}\right)
\end{aligned}
$$

here $\mathbf{d}=\left[d_{x}, d_{y}\right]^{T}$ is the dipole moment. Then (7) gives

$$
\begin{aligned}
& \hat{f}(x, q) \\
& =d_{x} \frac{\partial}{\partial x} \delta\left(x-x_{0}\right) \int_{-\infty}^{\infty} \delta\left(y-y_{0}\right) \mathrm{e}^{-\mathrm{i} q y} \mathrm{~d} y \\
& \quad+d_{y} \delta\left(x-x_{0}\right) \int_{-\infty}^{\infty} \frac{\partial}{\partial y} \delta\left(y-y_{0}\right) \mathrm{e}^{-\mathrm{i} q y} \mathrm{~d} y \\
& =d_{x} \frac{\partial}{\partial x} \delta\left(x-x_{0}\right) \mathrm{e}^{-\mathrm{i} q y_{0}}
\end{aligned}
$$

$$
\begin{aligned}
& -d_{y} \delta\left(x-x_{0}\right) \int_{-\infty}^{\infty} \delta\left(y-y_{0}\right)(-\mathrm{i} q) \mathrm{e}^{-\mathrm{i} q y} \mathrm{~d} y \\
= & d_{x} \frac{\partial}{\partial x} \delta\left(x-x_{0}\right) \mathrm{e}^{-\mathrm{i} q y_{0}}+\mathrm{i} q d_{y} \delta\left(x-x_{0}\right) \mathrm{e}^{-\mathrm{i} q y_{0}} .
\end{aligned}
$$

Next, (24) implies

$$
\begin{aligned}
I_{q}= & \int_{a}^{\infty} \hat{f}(\mathrm{~s}, q) \mathrm{e}^{-k_{0} \nu_{m} s} \mathrm{~d} s \\
= & d_{x} \mathrm{e}^{-\mathrm{i} q y_{0}} \int_{a}^{\infty} \frac{\partial}{\partial s} \delta\left(s-x_{0}\right) \mathrm{e}^{-k_{0} \nu_{m} s} \mathrm{~d} s \\
& +\mathrm{i} q d_{y} \mathrm{e}^{-\mathrm{i} q y_{0}} \int_{a}^{\infty} \delta\left(s-x_{0}\right) \mathrm{e}^{-k_{0} \nu_{m} s} \mathrm{~d} s \\
= & -d_{x} \mathrm{e}^{-\mathrm{i} q y_{0}} \int_{a}^{\infty} \delta\left(s-x_{0}\right)\left(-k_{0} v_{m}\right) \mathrm{e}^{-k_{0} \nu_{m} s} \mathrm{~d} s \\
& +\mathrm{i} q d_{y} \mathrm{e}^{-\mathrm{i} q y_{0}} \mathrm{e}^{-k_{0} v_{m} x_{0}} \\
= & d_{x} \mathrm{e}^{-\mathrm{i} q y_{0}} k_{0} v_{m} \mathrm{e}^{-k_{0} \nu_{m} x_{0}}+\mathrm{i} q d_{y} \mathrm{e}^{-\mathrm{i} q y_{0}} \mathrm{e}^{-k_{0} \nu_{m} x_{0}} \\
= & \left(d_{x} k_{0} v_{m}+\mathrm{i} d_{y} q\right) \mathrm{e}^{-k_{0} \nu_{m} x_{0}} \mathrm{e}^{-\mathrm{i} q y_{0} .} .
\end{aligned}
$$

If we take $x_{0}=d_{0}$ and $y_{0}=0$ (the typical case), this becomes

$$
I_{q}=\left(d_{x} k_{0} v_{m}+\mathrm{i} d_{y} q\right) \mathrm{e}^{-v_{m} d_{0}},
$$

which, after the changes of variables $p=q / k_{0}$ and $k_{0}=\gamma / a$, becomes

$$
\begin{aligned}
& I_{p} \\
& = \begin{cases}\mathrm{i} \frac{\gamma}{a}\left(d_{x} \sqrt{1-p^{2}}+d_{y} p\right) \mathrm{e}^{-\mathrm{i} \gamma\left(d_{0} / a\right) \sqrt{1-p^{2}}} & \text { if } 0 \leq p \leq 1, \\
\frac{\gamma}{a}\left(d_{x} \sqrt{p^{2}-1}+\mathrm{i} d_{y} p\right) \mathrm{e}^{-\gamma\left(d_{0} / a\right) \sqrt{p^{2}-1}} & \text { if } 1 \leq p<\infty .\end{cases}
\end{aligned}
$$

From here the triangle inequality implies that $I_{p}$ satisfies bound (47) with

$$
\begin{aligned}
& B(p ; \gamma) \\
& \equiv \begin{cases}\frac{\gamma}{a}\left(\left|d_{x}\right| \sqrt{1-p^{2}}+\left|d_{y}\right| p\right) & \text { for } 0 \leq p \leq 1, \\
\frac{\gamma}{a}\left(\left|d_{x}\right| \sqrt{p^{2}-1}+\left|d_{y}\right| p\right) & \text { for } 1 \leq p<\infty .\end{cases}
\end{aligned}
$$

Finally, the same computations as those leading up to (A.25) imply that quadrupole, octopole, and higher-order distributional sources satisfy equations similar to (A.25) with higher-order powers of $p$ and $\gamma$. Therefore, as discussed in Remark 9, such sources satisfy Theorems 6 and 8.

A.3. Proof of Theorem 6. We will prove this theorem in several steps. Essentially, our goal is to bound the integrand $L_{\delta}(p ; \gamma)$ from above by a function that is integrable and independent of $\delta$. We begin by finding a lower bound on $\left|g_{\delta}(p ; \gamma)\right|$; in 
particular, we note that (31) and the reverse triangle inequality imply that

$$
\begin{aligned}
& \left|g_{\delta}(p ; \gamma)\right| \\
& \quad \geq|| v_{s}-\left.(1+\mathrm{i} \delta) v_{m}\right|^{2}-\left|v_{s}+(1+\mathrm{i} \delta) v_{m}\right|^{2} \mathrm{e}^{-2 \gamma v_{s}^{\prime}} \mid
\end{aligned}
$$

In the next lemma, we provide a lower bound on the first term in (A.27).

Lemma A.1. Suppose $\gamma>\gamma_{*}$ and $\delta>0$; then

$$
\begin{aligned}
& \left|v_{s}-(1+\mathrm{i} \delta) v_{m}\right|^{2} \\
& \quad \geq \begin{cases}1 & \text { for } 0 \leq p \leq 1, \\
\left(\sqrt{p^{2}+1}-\sqrt{p^{2}-1}\right)^{2} & \text { for } 1 \leq p<\infty .\end{cases}
\end{aligned}
$$

Proof. We have

$$
\left|v_{s}-(1+\mathrm{i} \delta) v_{m}\right|^{2} \geq\left|\operatorname{Re}\left[v_{s}-(1+\mathrm{i} \delta) v_{m}\right]\right|^{2}
$$

If $0 \leq p \leq 1$, then

$$
\begin{aligned}
& \left|\operatorname{Re}\left[v_{s}-(1+\mathrm{i} \delta) v_{m}\right]\right| \\
& \quad=\sqrt{\frac{\left(p^{2}+1\right)+\sqrt{\left(p^{2}+1\right)^{2}+\delta^{2}}}{2}}+\delta \sqrt{1-p^{2}}
\end{aligned}
$$

$\geq 1$.

If $1 \leq p<\infty$, then

$$
\begin{aligned}
\mid \operatorname{Re} & {\left[v_{s}-(1+\mathrm{i} \delta) v_{m}\right] \mid } \\
& =\sqrt{\frac{\left(p^{2}+1\right)+\sqrt{\left(p^{2}+1\right)^{2}+\delta^{2}}}{2}}-\sqrt{p^{2}-1} \\
& \geq \sqrt{p^{2}+1}-\sqrt{p^{2}-1}
\end{aligned}
$$

Squaring both sides of (A.30) and (A.31) and utilizing (A.29) give us the desired result.

In the next lemma, we provide upper bounds on the second term in (A.27).

Lemma A.2. Suppose $\gamma>\gamma_{*}$; then there is a constant $0<C<$ 1 such that

$$
\left|v_{s}+(1+\mathrm{i} \delta) v_{m}\right|^{2} \mathrm{e}^{-2 \gamma v_{s}^{\prime}} \leq \begin{cases}C & \text { for } 0 \leq p \leq 1,0<\delta \leq 0.4 \\ (1+3 \sqrt{\delta})\left(\sqrt{p^{2}+1}+\sqrt{p^{2}-1}\right)^{2} \mathrm{e}^{-2 \gamma \sqrt{p^{2}+1}} & \text { for } 1 \leq p<\infty, 0<\delta \leq 1\end{cases}
$$

Proof. The triangle inequality, the assumptions that $\gamma>\gamma_{*}$ and $\delta>0$, and the bound

$$
\nu_{s}^{\prime}=\sqrt{\frac{\left(p^{2}+1\right)+\sqrt{\left(p^{2}+1\right)^{2}+\delta^{2}}}{2}} \geq \sqrt{p^{2}+1}
$$

imply that

$$
\begin{aligned}
\mid \nu_{s} & +(1+\mathrm{i} \delta) \nu_{m} \mid \mathrm{e}^{-\gamma v_{s}^{\prime}} \\
& \leq\left(\left|\nu_{s}\right|+\sqrt{1+\delta^{2}}\left|\nu_{m}\right|\right) \mathrm{e}^{-\gamma_{*} \sqrt{p^{2}+1}} .
\end{aligned}
$$

For $0 \leq p \leq 1$ and $0<\delta \leq 0.4$, the definitions of $v_{s}$ and $\nu_{m}$ from (15) and (19), respectively, imply that the above bound becomes

$$
\begin{aligned}
\mid \nu_{s} & +(1+\mathrm{i} \delta) v_{m} \mid \mathrm{e}^{-\gamma v_{s}^{\prime}} \\
& \leq\left\{\left[\left(p^{2}+1\right)^{2}+\delta^{2}\right]^{1 / 4}+\sqrt{1+\delta^{2}} \sqrt{1-p^{2}}\right\} \\
\cdot & \mathrm{e}^{-\gamma_{*} \sqrt{p^{2}+1}} \leq\left[\left(4+\delta^{2}\right)^{1 / 4}+\sqrt{1+\delta^{2}}\right] \mathrm{e}^{-\gamma_{*}} \\
& \leq\left[\left(4+0.4^{2}\right)^{1 / 4}+\sqrt{1+0.4^{2}}\right] \mathrm{e}^{-\gamma_{*}} \approx 0.9813 .
\end{aligned}
$$

Therefore, if we take $C=0.99$, we have

$$
\left|v_{s}+(1+\mathrm{i} \delta) v_{m}\right|^{2} \mathrm{e}^{-2 \gamma v_{s}^{\prime}} \leq C
$$

Now we consider the case $1 \leq p<\infty$. The triangle inequality, the bound in (A.33), and the definitions of $\nu_{s}$ and $v_{m}$ for $p \geq 1$ (see (15) and (19), resp.), imply that

$$
\begin{aligned}
\mid v_{s} & +(1+\mathrm{i} \delta) \nu_{m} \mid \mathrm{e}^{-\gamma v_{s}^{\prime}} \leq\left(\left|v_{s}\right|+\sqrt{1+\delta^{2}}\left|\nu_{m}\right|\right) \\
\cdot & \mathrm{e}^{-\gamma \sqrt{p^{2}+1}} \\
& =\left\{\left[\left(p^{2}+1\right)^{2}+\delta^{2}\right]^{1 / 4}+\sqrt{1+\delta^{2}} \sqrt{p^{2}-1}\right\} \\
\cdot & \mathrm{e}^{-\gamma \sqrt{p^{2}+1}} .
\end{aligned}
$$

Applying the bound $(x+y)^{r} \leq x^{r}+y^{r}$ for $r=1 / 4$ and $r=1 / 2$ to the right-hand side of the above inequality implies

$$
\begin{aligned}
\mid \nu_{s} & +(1+\mathrm{i} \delta) \nu_{m} \mid \mathrm{e}^{-\gamma v_{s}^{\prime}} \\
\quad \leq & {\left[\sqrt{p^{2}+1}+\sqrt{\delta}+(1+\delta) \sqrt{p^{2}-1}\right] \mathrm{e}^{-\gamma \sqrt{p^{2}+1}} . }
\end{aligned}
$$


Squaring the term in brackets on right-hand side of the above expression gives

$$
\begin{aligned}
& {\left[\sqrt{p^{2}+1}+\sqrt{\delta}+(1+\delta) \sqrt{p^{2}-1}\right]^{2}} \\
& =\left(\sqrt{p^{2}+1}+\sqrt{p^{2}-1}\right)^{2}+Q_{\delta}(p)
\end{aligned}
$$

where

$$
\begin{aligned}
Q_{\delta} & (p) \equiv 2\left(\sqrt{p^{2}+1}+\sqrt{p^{2}-1}\right)(\sqrt{\delta} \\
+ & \left.\delta \sqrt{p^{2}-1}\right)+\left(\sqrt{\delta}+\delta \sqrt{p^{2}-1}\right)^{2} \\
& =\sqrt{\delta}\left[2\left(\sqrt{p^{2}+1}+\sqrt{p^{2}-1}\right)\right. \\
& \left.\cdot\left(1+\sqrt{\delta} \sqrt{p^{2}-1}\right)+\sqrt{\delta}\left(1+\sqrt{\delta} \sqrt{p^{2}-1}\right)^{2}\right] .
\end{aligned}
$$

For $p \geq 1$ and $0<\delta \leq 1$, we have the bound

$$
1+\sqrt{\delta} \sqrt{p^{2}-1} \leq \sqrt{p^{2}+1}+\sqrt{p^{2}-1}
$$

Using this bound in (A.40) gives

$$
\begin{aligned}
Q_{\delta}(p) & \leq \sqrt{\delta}(2+\sqrt{\delta})\left(\sqrt{p^{2}+1}+\sqrt{p^{2}-1}\right)^{2} \\
& \leq 3 \sqrt{\delta}\left(\sqrt{p^{2}+1}+\sqrt{p^{2}-1}\right)^{2}
\end{aligned}
$$

Combining (A.38), (A.39), and (A.42) gives us the second bound in (A.32).

We are now ready to give lower bounds on $\left|g_{\delta}(p ; \gamma)\right|$.

Lemma A.3. Suppose $\gamma>\gamma_{*}$; then there exist $\delta_{\gamma}$ satisfying $0<$ $\delta_{\gamma} \leq 0.4$ and a positive constant $C$ such that, for $0<\delta \leq \delta_{\gamma}$,

$$
\left|g_{\delta}(p ; \gamma)\right| \geq \begin{cases}C & \text { for } 0 \leq p \leq 1, \\ \frac{\left|g_{0}(p ; \gamma)\right|}{2} & \text { for } 1 \leq p<\infty\end{cases}
$$

Proof. If $0 \leq p \leq 1$ and $0<\delta \leq 0.4$, then (A.27) and Lemmas A.1 and A. 2 imply that there is a constant $0<C<1$ such that

$$
\begin{aligned}
\left|g_{\delta}(p ; \gamma)\right| \geq \mid & \left|\nu_{s}-(1+\mathrm{i} \delta) \nu_{m}\right|^{2} \\
& \quad-\left|\nu_{s}+(1+\mathrm{i} \delta) \nu_{m}\right|^{2} \mathrm{e}^{-2 \gamma v_{s}^{\prime}} \geq 1-C \\
> & 0 .
\end{aligned}
$$

This gives us first part of (A.43).

We now assume $1 \leq p<\infty$. First, note that $g_{0}(p ; \gamma)>$ 0 for all $p \geq 1$ because it has no zeros by Lemma 5 and $g_{0}(1 ; \gamma)>0$. Then (35), (A.27), and Lemmas A.1 and A.2 imply that

$$
\begin{aligned}
\mid g_{\delta} & (p ; \gamma) \mid \\
\geq & \left(\sqrt{p^{2}+1}-\sqrt{p^{2}-1}\right)^{2} \\
& \quad-(1+3 \sqrt{\delta})\left(\sqrt{p^{2}+1}+\sqrt{p^{2}-1}\right)^{2} \mathrm{e}^{-2 \gamma \sqrt{p^{2}+1}} \\
= & \left|g_{0}(p ; \gamma)\right| \\
& -3 \sqrt{\delta}\left(\sqrt{p^{2}+1}+\sqrt{p^{2}-1}\right)^{2} \mathrm{e}^{-2 \gamma \sqrt{p^{2}+1}}
\end{aligned}
$$

for all $0<\delta \leq 1$. Therefore,

$$
\begin{aligned}
& \left|g_{\delta}\right|-\frac{\left|g_{0}\right|}{2} \\
& \geq \frac{\left|g_{0}\right|}{2}-3 \sqrt{\delta}\left(\sqrt{p^{2}+1}+\sqrt{p^{2}-1}\right)^{2} \mathrm{e}^{-2 \gamma \sqrt{p^{2}+1}} \\
& =\frac{1}{2}\left(\sqrt{p^{2}+1}-\sqrt{p^{2}-1}\right)^{2} \\
& \quad-\left(\frac{1}{2}+3 \sqrt{\delta}\right)\left(\sqrt{p^{2}+1}+\sqrt{p^{2}-1}\right)^{2} \mathrm{e}^{-2 \gamma \sqrt{p^{2}+1}}
\end{aligned}
$$

for all $0<\delta \leq 1$. From (A.47), for all $0<\delta \leq 0.4$, we have

$$
\begin{aligned}
\left|g_{\delta}\right|-\frac{\left|g_{0}\right|}{2} \geq \frac{1}{2} & \left(\sqrt{p^{2}+1}-\sqrt{p^{2}-1}\right)^{2} \\
& -\left(\frac{1}{2}+3 \sqrt{0.4}\right) \\
& \cdot\left(\sqrt{p^{2}+1}+\sqrt{p^{2}-1}\right)^{2} \\
& \cdot \mathrm{e}^{-2 \gamma \sqrt{p^{2}+1}} .
\end{aligned}
$$

Thanks to the exponential decay in the second term on the right-hand side of (A.48), there exists $\widetilde{p}_{\gamma} \geq 1$ such that the expression on the right-hand side of (A.48) is strictly positive for all $p \geq \widetilde{p}_{\gamma}$. Therefore,

$$
\begin{aligned}
&\left|g_{\delta}(p ; \gamma)\right| \geq \frac{\left|g_{0}(p ; \gamma)\right|}{2} \\
& \forall p \geq \widetilde{p}_{\gamma} \text { and all } 0<\delta \leq 0.4 .
\end{aligned}
$$

Finally, we consider $1 \leq p \leq \widetilde{p}_{\gamma}$. Because both terms on the right-hand side of (A.46) are continuous, we may define

$$
\begin{aligned}
& m_{\gamma} \equiv \min _{1 \leq p \leq \tilde{p}_{\gamma}} \frac{\left|g_{0}(p ; \gamma)\right|}{2} \\
& M_{\gamma} \equiv \max _{1 \leq p \leq \tilde{p}_{\gamma}}\left[3\left(\sqrt{p^{2}+1}+\sqrt{p^{2}-1}\right)^{2} \mathrm{e}^{-2 \gamma \sqrt{p^{2}+1}}\right]
\end{aligned}
$$


because $\gamma>\gamma_{*}, m_{\gamma}>0$ by Lemma 5 . Hence (A.46) becomes

$$
\left|g_{\delta}(p ; \gamma)\right|-\frac{\left|g_{0}(p ; \gamma)\right|}{2} \geq m_{\gamma}-\sqrt{\delta} M_{\gamma}
$$

which is nonnegative if we take

$$
\delta \leq\left(\frac{m_{\gamma}}{M_{\gamma}}\right)^{2} .
$$

Therefore

$$
\begin{aligned}
\left|g_{\delta}(p ; \gamma)\right| \geq & \frac{\left|g_{0}(p ; \gamma)\right|}{2} \\
& \forall 1 \leq p \leq \tilde{p}_{\gamma} \text { and all } 0<\delta \leq\left(\frac{m_{\gamma}}{M_{\gamma}}\right)^{2} .
\end{aligned}
$$

We define $\delta_{\gamma} \equiv \min \left\{0.4, m_{\gamma}^{2} / M_{\gamma}^{2}\right\}$; then (A.49) and (A.53) imply that

$$
\begin{aligned}
\left|g_{\delta}(p ; \gamma)\right| \geq \frac{\left|g_{0}(p ; \gamma)\right|}{2} & \\
& \forall 1 \leq p<\infty, 0<\delta \leq \delta_{\gamma} .
\end{aligned}
$$

This completes the proof.

Recalling that our ultimate goal is to prove Theorem 6, in the next lemma we derive upper bounds on $M_{\delta}(p ; \gamma)$, defined in (38).

Lemma A.4. Suppose $\gamma>\gamma_{*}$ and $0<\delta \leq \delta_{\gamma}$, where $\delta_{\gamma}$ is defined in Lemma A.3. Then there exists a constant $C_{\gamma}>0$ such that

$$
\begin{aligned}
& \left|M_{\delta}(p ; \gamma)\right| \\
& \quad \leq C_{\gamma}\left\{\left[\left(p^{2}+1\right)^{2}+1\right]^{1 / 4}+\sqrt{2} \sqrt{\left|p^{2}-1\right|}\right\}^{2} \\
& \quad \cdot\left[\sqrt{\left(p^{2}+1\right)^{2}+1}+p^{2}\right]
\end{aligned}
$$

for all $0 \leq p<\infty$ and all $0<\delta \leq \delta_{\gamma}$.

Proof. By the triangle inequality, we may derive upper bounds on each term of $\left|M_{\delta}(p ; \gamma)\right|$ individually.

(1) For the term outside of the braces in (38), we have

$$
\begin{aligned}
\left|v_{s}-(1+\mathrm{i} \delta) \nu_{m}\right| \leq & \left|v_{s}\right|+|1+\mathrm{i} \delta|\left|\nu_{m}\right| \\
= & {\left[\left(p^{2}+1\right)^{2}+\delta^{2}\right]^{1 / 4} } \\
& +\sqrt{1+\delta^{2}} \sqrt{\left|p^{2}-1\right|} \\
\leq & {\left[\left(p^{2}+1\right)^{2}+\delta_{\gamma}^{2}\right]^{1 / 4} } \\
& +\sqrt{1+\delta_{\gamma}^{2}} \sqrt{\left|p^{2}-1\right|} .
\end{aligned}
$$

Therefore, for all $p \geq 0$ and all $0<\delta \leq \delta_{\gamma}<1$, we have

$$
\begin{aligned}
& \mid \nu_{s}-\left.(1+\mathrm{i} \delta) v_{m}\right|^{2} \\
& \quad \leq\left\{\left[\left(p^{2}+1\right)^{2}+\delta_{\gamma}^{2}\right]^{1 / 4}+\sqrt{1+\delta_{\gamma}^{2}} \sqrt{\left|p^{2}-1\right|}\right\}^{2} \\
& \quad \leq\left\{\left[\left(p^{2}+1\right)^{2}+1\right]^{1 / 4}+\sqrt{2} \sqrt{\left|p^{2}-1\right|}\right\}^{2} .
\end{aligned}
$$

(2) For the first term in brackets in (38), we have, thanks to (A.33),

$$
\left|\frac{1-\mathrm{e}^{-2 \gamma v_{s}^{\prime}}}{2 v_{s}^{\prime}}\right|=\frac{1-\mathrm{e}^{-2 \gamma v_{s}^{\prime}}}{2 v_{s}^{\prime}} \leq \frac{1-\mathrm{e}^{-2 \gamma \sqrt{p^{2}+1}}}{2 \sqrt{p^{2}+1}}
$$

Because the above function is continuous for $p \in$ $[0, \infty)$ and tends to 0 as $p \rightarrow \infty$, it attains its maximum value on $[0, \infty)$. Thus there is a constant $C_{\gamma}>0$ such that

$$
\left|\frac{1-\mathrm{e}^{-2 \gamma v_{s}^{\prime}}}{2 \nu_{s}^{\prime}}\right| \leq C_{\gamma}
$$

(3) Using our result from item (2) and (18), we find that the second term in brackets in (38) satisfies

$$
\begin{aligned}
& |R|^{2} \mathrm{e}^{-2 \gamma v_{s}^{\prime}}\left|\frac{1-\mathrm{e}^{-2 \gamma v_{s}^{\prime}}}{2 v_{s}^{\prime}}\right| \\
& \quad \leq C_{\gamma} \frac{\left|v_{s}+(1+\mathrm{i} \delta) \nu_{m}\right|^{2} \mathrm{e}^{-2 \gamma v_{s}^{\prime}}}{\left|\nu_{s}-(1+\mathrm{i} \delta) \nu_{m}\right|^{2}},
\end{aligned}
$$

where $C_{\gamma}$ is the constant from (A.59). Applying the bounds from (A.28) and (A.32) as well as the bounds $\gamma>\gamma_{*}$ and $\delta \leq \delta_{\gamma}<1$ to the above expression gives

$$
|R|^{2} \mathrm{e}^{-2 \gamma v_{s}^{\prime}}\left|\frac{1-\mathrm{e}^{-2 \gamma v_{s}^{\prime}}}{2 \nu_{s}^{\prime}}\right|
$$

$$
\leq \begin{cases}C_{\gamma} & \text { for } 0 \leq p \leq 1, \\ C_{\gamma} \frac{4\left(\sqrt{p^{2}+1}+\sqrt{p^{2}-1}\right)^{2} \mathrm{e}^{-2 \gamma_{*} \sqrt{p^{2}+1}}}{\left(\sqrt{p^{2}+1}-\sqrt{p^{2}-1}\right)^{2}} & \text { for } 1 \leq p<\infty .\end{cases}
$$

The function on the right-hand side of the above inequality is continuous as a function of $p$ for $p \in$ $[1, \infty)$ and decays to 0 as $p \rightarrow \infty$. Thus it attains its maximum value on $[1, \infty$ ) (this maximum value is independent of $\gamma$ ); this and (A.61) imply that there is a constant $C_{\gamma}>0$ such that

$$
|R|^{2} \mathrm{e}^{-2 \gamma v_{s}^{\prime}}\left|\frac{1-\mathrm{e}^{-2 \gamma v_{s}^{\prime}}}{2 v_{s}^{\prime}}\right| \leq C_{\gamma}
$$

for all $p \geq 0$ and all $0<\delta \leq \delta_{\gamma}$. 
(4) For the last term in (38), we have

$$
\begin{gathered}
\left|\mathrm{e}^{-2 \gamma v_{s}^{\prime}} \operatorname{Im}\left[\bar{R} \mathrm{e}^{2 \mathrm{i} \gamma v_{s}^{\prime \prime}}\left(\frac{1-\mathrm{e}^{-2 \mathrm{i} \gamma \nu_{s}^{\prime \prime}}}{2 \nu_{s}^{\prime \prime}}\right)\right]\right| \\
\leq \mathrm{e}^{-2 \gamma v_{s}^{\prime}}|R|\left|\frac{1-\mathrm{e}^{-2 \mathrm{i} \gamma \nu_{s}^{\prime \prime}}}{2 v_{s}^{\prime \prime}}\right| .
\end{gathered}
$$

Arguments similar to those in item (3) can be used to show that there is a positive constant $C$ such that

$$
|R| \mathrm{e}^{-\gamma \nu_{s}^{\prime}} \leq C
$$

Because

$$
v_{s}^{\prime \prime}=\frac{\delta}{2 v_{s}^{\prime}}
$$

the function

$$
\mathrm{e}^{-\gamma v_{s}^{\prime}}\left|\frac{1-\mathrm{e}^{-2 \gamma \mathrm{i} \nu_{s}^{\prime \prime}}}{2 \nu_{s}^{\prime \prime}}\right|
$$

is continuous for $0 \leq \delta \leq \delta_{\gamma}$ and $0 \leq p<\infty$ (after modification at $\delta=0$ ). Moreover, this function goes to 0 as $p \rightarrow \infty$, so it attains its maximum value. This implies that there is a constant $C_{\gamma}>0$ such that

$$
\mathrm{e}^{-\gamma v_{s}^{\prime}}\left|\frac{1-\mathrm{e}^{-2 \gamma \mathrm{i} v_{s}^{\prime \prime}}}{2 \nu_{s}^{\prime \prime}}\right| \leq C_{\gamma}
$$

Inserting (A.64) and (A.67) into (A.63) implies that there is a constant $C_{\gamma}>0$ such that

$$
\begin{gathered}
\left|\mathrm{e}^{-2 \gamma v_{s}^{\prime}} \operatorname{Im}\left[\bar{R} \mathrm{e}^{2 \mathrm{i} \gamma v_{s}^{\prime \prime}}\left(\frac{1-\mathrm{e}^{-2 \mathrm{i} \gamma v_{s}^{\prime \prime}}}{2 \nu_{s}^{\prime \prime}}\right)\right]\right| \\
\leq \mathrm{e}^{-2 \gamma v_{s}^{\prime}}|R|\left|\frac{1-\mathrm{e}^{-2 \mathrm{i} \gamma v_{s}^{\prime \prime}}}{2 v_{s}^{\prime \prime}}\right| \leq C_{\gamma}
\end{gathered}
$$

for all $0 \leq p<\infty$ and all $0<\delta \leq \delta_{\gamma}$.

Using the bound

$$
\left.| \pm| v_{s}\right|^{2}+\left.p^{2}|\leq| v_{s}\right|^{2}+p^{2} \leq \sqrt{\left(p^{2}+1\right)^{2}+1}+p^{2}
$$

which holds for all $p \geq 1$ and all $\delta \leq \delta_{\gamma}<1$, as well as the bounds (A.57), (A.59), (A.62), and (A.68) in (38) gives

$$
\begin{aligned}
& \left|M_{\delta}(p ; \gamma)\right| \\
& \quad \leq C_{\gamma}\left\{\left[\left(p^{2}+1\right)^{2}+1\right]^{1 / 4}+\sqrt{2} \sqrt{\left|p^{2}-1\right|}\right\}^{2} \\
& \quad \cdot\left[\sqrt{\left(p^{2}+1\right)^{2}+1}+p^{2}\right],
\end{aligned}
$$

for some positive constant $C_{\gamma}$.
We are finally ready to complete the proof of Theorem 6 . First, we split the integral in (36) to obtain

$$
\begin{aligned}
\int_{0}^{\infty} L_{\delta}(p ; \gamma) \mathrm{d} p= & \int_{0}^{1} L_{\delta}(p ; \gamma) \mathrm{d} p \\
& +\int_{1}^{\infty} L_{\delta}(p ; \gamma) \mathrm{d} p
\end{aligned}
$$

We will focus on each integral separately. For $0 \leq p \leq 1$, we use the bounds from Lemma A.3, (A.55), and (39) in (37) to obtain the bound

$$
\begin{aligned}
& L_{\delta}(p ; \gamma) \\
& \quad \leq C_{\gamma}\left\{\left[\left(p^{2}+1\right)^{2}+1\right]^{1 / 4}+\sqrt{2} \sqrt{\left|p^{2}-1\right|}\right\}^{2} \\
& \quad \cdot\left[\sqrt{\left(p^{2}+1\right)^{2}+1}+p^{2}\right],
\end{aligned}
$$

which holds for all $0 \leq p \leq 1$ and $0<\delta \leq \delta_{\gamma}$. The function on the right-hand side of the above inequality is continuous for $p \in[0,1]$, so it attains its maximum value. Thus there is a positive constant $C_{\gamma}$ such that

$$
\int_{0}^{1} L_{\delta}(p ; \gamma) \leq C_{\gamma} \quad \forall 0 \leq p \leq 1 \text { and all } 0<\delta \leq \delta_{\gamma} .
$$

Similarly, for $p \geq 1$, the bounds from Lemmas A.3, (A.55), and (39) imply that there is a positive constant $C_{\gamma}$ such that

$$
\begin{aligned}
& L_{\delta}(p ; \gamma) \leq C_{\gamma} \\
& \quad \cdot \frac{\mathrm{e}^{-2 \gamma\left(d_{0} / a-1\right) \sqrt{p^{2}-1}}}{\left|g_{0}(p ; \gamma)\right|^{2}}\left\{\left[\left(p^{2}+1\right)^{2}+1\right]^{1 / 4}\right. \\
& \left.\quad+\sqrt{2} \sqrt{\left|p^{2}-1\right|}\right\}^{2}\left[\sqrt{\left(p^{2}+1\right)^{2}+1}+p^{2}\right]
\end{aligned}
$$

this bound holds for all $1 \leq p<\infty$ and all $0<\delta \leq \delta_{\gamma}$. Note from (35) that $\left|g_{0}\right| \rightarrow 0$ as $p \rightarrow \infty$ at an algebraic (i.e., nonexponential) rate. Because $d_{0} / a>1$, the exponential term in the numerator in (A.74) defeats the nonexponential terms in the braces and in $\left|g_{0}\right|^{2}$; in other words, there is a positive constant $C_{\gamma}>0$ such that

$$
\begin{aligned}
\int_{1}^{\infty} L_{\delta}(p ; \gamma) \leq C_{\gamma} & \\
& \forall 1 \leq p<\infty \text { and all } 0<\delta \leq \delta_{\gamma} .
\end{aligned}
$$

Using (A.73) and (A.75) in (36) gives

$$
E_{\delta}(a) \leq C_{\gamma} \quad \forall 0<\delta \leq \delta_{\gamma} .
$$

This completes the proof of the theorem.

A.4. Proof of Theorem 8. We begin by taking care of an important technicality. Recall from Section A.3 that $\widetilde{p}_{\gamma}$ was chosen so that the expression on the right-hand side of (A.48) 


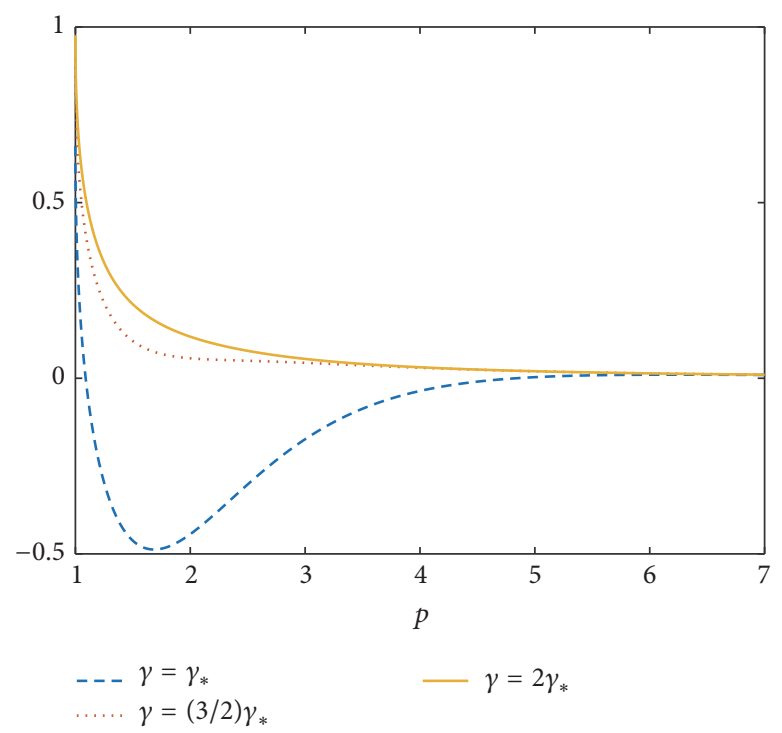

(a)

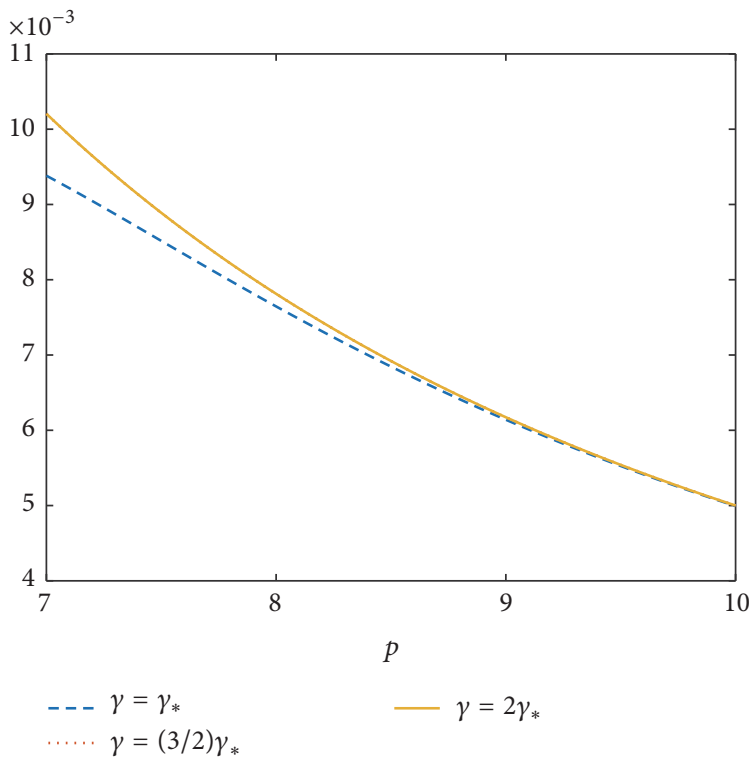

(b)

FIGURE 14: This figure contains a plot of the expression on the right-hand side of (A.48). In both plots, the blue dashed curve corresponds to $\gamma=\gamma_{*}$, the red dotted curve corresponds to $\gamma=(3 / 2) \gamma_{*}$, and the yellow solid curve corresponds to $\gamma=2 \gamma_{*}$. In particular, we have plotted the expression in (A.48) over the intervals (a) $1 \leq p \leq 7$ and (b) $7 \leq p \leq 10$.

is strictly positive for $p \geq \tilde{p}_{\gamma}$. Because of this choice, $\delta_{\gamma}$ depends on $\gamma$ in a nontrivial way-see (A.53). However, there is $\widetilde{\gamma}>0$ such that if $\gamma \geq \tilde{\gamma}$, the right-hand side of (A.48) is positive for all $p \geq 1$ (thanks to the exponential decay in $\gamma$ of the last term in (A.48)). Thus, for $\gamma \geq \tilde{\gamma}$, (A.48) immediately implies that there is $\delta_{0}$, independent of $\gamma$, such that $0<\delta_{0} \leq 0.4$ and

$$
\left|g_{\delta}(p ; \gamma)\right| \geq \frac{g_{0}(p ; \gamma)}{2}
$$

for all $p \geq 1$, all $0<\delta \leq \delta_{0}$, and all $\gamma \geq \tilde{\gamma}$. To visualize this, in Figure 14 we plot the expression on the right-hand side of (A.48) as a function of $p$ for different values of $\gamma$. Figure 14(a) is a plot over the interval $1 \leq p \leq 7$ while Figure 14(b) is a plot over the interval $7 \leq p \leq 10$; in addition, the blue dashed curve is for $\gamma=\gamma_{*}$, the red dotted curve is for $\gamma=(3 / 2) \gamma_{*}$, and the yellow solid curve is for $\gamma=2 \gamma_{*}$ (the $\gamma=(3 / 2) \gamma_{*}$ and $\gamma=2 \gamma_{*}$ curves overlap in Figure 14(b). In Figure 14(a), we note that the expression is negative for some values of $p$ if $\gamma=\gamma_{*}$; however, if $\gamma=(3 / 2) \gamma_{*}$ or $\gamma=2 \gamma_{*}$, the curve is always positive. Therefore, for the remainder of this proof, we will assume $0<\delta \leq \delta_{0}$ and $\gamma \geq 2 \gamma_{*}$.

The Fourier inversion theorem, the triangle inequality, and (14) imply that

$$
\begin{aligned}
\left|V_{c}(x, y)\right| & =\left|\frac{1}{2 \pi} \int_{-\infty}^{\infty} A_{q} \mathrm{e}^{k_{0} v_{c} x} \mathrm{e}^{\mathrm{i} q y} \mathrm{~d} q\right| \\
& \leq \frac{1}{2 \pi} \int_{-\infty}^{\infty}\left|A_{q}\right| \mathrm{e}^{k_{0} v_{c}^{\prime} x} \mathrm{~d} q \\
& =\frac{1}{\pi} \int_{0}^{\infty}\left|A_{q}\right| \mathrm{e}^{k_{0} v_{c}^{\prime} x} \mathrm{~d} q .
\end{aligned}
$$

Using (21)-(23) and (31) and making the change of variables $p=q / k_{0}$ in the above integral gives

$$
\begin{aligned}
& \left|V_{c}(x, y)\right| \\
& \quad \leq \frac{2|1+\mathrm{i} \delta|}{\pi} \int_{0}^{\infty} \frac{\left|I_{p}\right|\left|\nu_{s}\right| \mathrm{e}^{\gamma v_{m}^{\prime}} \mathrm{e}^{-\gamma v_{s}^{\prime}} \mathrm{e}^{k_{0} v_{c}^{\prime} x}}{\left|g_{\delta}(p ; \gamma)\right|} \mathrm{d} p .
\end{aligned}
$$

Case $1(0 \leq p \leq 1)$. In this case, the integral in (A.79) (restricted to $0 \leq p \leq 1$ ) is

$$
\int_{0}^{1} \frac{\left|I_{p}\right|\left|v_{s}\right| \mathrm{e}^{-\gamma v_{s}^{\prime}}}{\left|g_{\delta}(p ; \gamma)\right|} \mathrm{d} p .
$$

For $\gamma \geq 2 \gamma_{*}$ and $0<\delta \leq \delta_{0} \leq 1$, (A.33) and Lemmas A.3 and 7 imply that there is a constant $C>0$ such that the above integral is less than or equal to

$$
\begin{aligned}
& C \int_{0}^{1}\left|v_{s}\right| \mathrm{e}^{-\gamma v_{s}^{\prime}} \mathrm{d} p \leq C \int_{0}^{1}\left[\left(p^{2}+1\right)^{2}+1\right]^{1 / 4} \\
& \cdot \mathrm{e}^{-\gamma \sqrt{p^{2}+1}} \mathrm{~d} p=C \int_{0}^{1}\left[\left(p^{2}+1\right)^{2}+1\right]^{1 / 4} \\
& \cdot \mathrm{e}^{-\eta \gamma \sqrt{p^{2}+1}} \mathrm{e}^{-(1-\eta) \gamma \sqrt{p^{2}+1}} \mathrm{~d} p \\
& \leq C \mathrm{e}^{-\eta \gamma} \int_{0}^{1}\left[\left(p^{2}+1\right)^{2}+1\right]^{1 / 4} \mathrm{e}^{-(1-\eta) 2 \gamma * \sqrt{p^{2}+1}} \mathrm{~d} p .
\end{aligned}
$$

Because the integrand in (A.81) is continuous, it is bounded above by a constant (independent of $p, \delta$, and $\gamma$ ). Thus (A.81) implies that there is $C_{\eta}>0$ such that

$$
\int_{0}^{1} \frac{\left|I_{p}\right|\left|\nu_{s}\right| \mathrm{e}^{-\gamma \nu_{s}^{\prime}}}{|g|} \mathrm{d} p \leq C_{\eta} \mathrm{e}^{-\eta \gamma} .
$$


Case $2(1 \leq p<\infty)$. In this case, the integral in (A.79) (restricted to $1 \leq p<\infty$ ) is

$$
\int_{1}^{\infty} \frac{\left|I_{p}\right|\left|v_{s}\right| \mathrm{e}^{\gamma \sqrt{p^{2}-1}} \mathrm{e}^{-\gamma v_{s}^{\prime}} \mathrm{e}^{k_{0} \sqrt{p^{2}-1} x}}{|g|} \mathrm{d} p .
$$

For $\gamma \geq 2 \gamma_{*}$ and $0<\delta \leq \delta_{0} \leq 1$, (A.33) and Lemmas A.3 and 7 imply that there is a constant $C>0$ such that the above integral is less than or equal to

$$
C \int_{1}^{\infty} \frac{\left[\left(p^{2}+1\right)^{2}+1\right]^{1 / 4} \mathrm{e}^{-\gamma\left(d_{0} / a-1\right)} \sqrt{p^{2}-1} \mathrm{e}^{-\gamma \sqrt{p^{2}+1}} \mathrm{e}^{k_{0}} \sqrt{p^{2}-1} x}{\left|g_{0}(p ; \gamma)\right|} \mathrm{d} p .
$$

From (35), the denominator of the above integrand is an increasing function of $\gamma$. Together with the fact that all of the exponential terms in the numerator attain their maximum values at $p=1$, this implies, for $\gamma \geq 2 \gamma_{*}$, that the above integral is bounded above by

$$
\begin{aligned}
& C \int_{1}^{\infty} \frac{\left[\left(p^{2}+1\right)^{2}+\delta^{2}\right]^{1 / 4} \mathrm{e}^{-\gamma \sqrt{p^{2}+1}}}{\left|g_{0}\left(p ; 2 \gamma_{*}\right)\right|} \mathrm{d} p \\
& \leq C \int_{1}^{\infty} \frac{\left[\left(p^{2}+1\right)^{2}+1\right]^{1 / 4} \mathrm{e}^{-\gamma \sqrt{p^{2}+1}}}{\left|g_{0}\left(p ; 2 \gamma_{*}\right)\right|} \mathrm{d} p \\
& =C \int_{1}^{\infty} \frac{\left[\left(p^{2}+1\right)^{2}+1\right]^{1 / 4} \mathrm{e}^{-\eta \gamma \sqrt{p^{2}+1}} \mathrm{e}^{-(1-\eta) \gamma \sqrt{p^{2}+1}}}{\left|g_{0}\left(p ; 2 \gamma_{*}\right)\right|} \mathrm{d} p \\
& \leq C \mathrm{e}^{-\sqrt{2} \eta \gamma} \int_{1}^{\infty} \frac{\left[\left(p^{2}+1\right)^{2}+1\right]^{1 / 4} \mathrm{e}^{-(1-\eta) 2 \gamma_{*}} \sqrt{p^{2}+1}}{\left|g_{0}\left(p ; 2 \gamma_{*}\right)\right|} \mathrm{d} p .
\end{aligned}
$$

Because $\left|g_{0}\left(p ; 2 \gamma_{*}\right)\right|$ has no roots (by Lemma 5) and tends to 0 as $p \rightarrow \infty$ only algebraically, the above integral converges. This implies that

$$
\int_{1}^{\infty} \frac{\left|I_{p}\right|\left|v_{s}\right| \mathrm{e}^{\gamma v_{m}^{\prime}} \mathrm{e}^{-\gamma v_{s}^{\prime}} \mathrm{e}^{k_{0} v_{c}^{\prime} x}}{|g|} \mathrm{d} p \leq C_{\eta} \mathrm{e}^{-\sqrt{2} \eta \gamma}
$$

for some $C_{\eta}>0$. Using (A.82) and (A.86) in (A.79) gives

$$
\left|V_{c}(x, y)\right| \leq C_{\eta} \mathrm{e}^{-\eta \gamma}=C_{\eta} \mathrm{e}^{-\eta k_{0} a} ;
$$

this bound holds for all $0<\eta<1, \gamma \geq 2 \gamma_{*}$, all $0<\delta \leq \delta_{0}$, and all $(x, y) \in \mathscr{C}$. Thus $V_{c}(x, y)$ goes to 0 exponentially as $k_{0} \rightarrow \infty$. This completes the proof of the theorem.

\section{A.5. Derivation of Helmholtz Equation from Maxwell Equa-} tions. When no charge source is present, the Maxwell equations are

$$
\begin{aligned}
& \nabla \cdot \mathbf{D}=0, \\
& \nabla \times \mathbf{E}=-\frac{\partial \mathbf{B}}{\partial t}, \\
& \nabla \cdot \mathbf{B}=0, \\
& \nabla \times \mathbf{H}=\frac{\partial \mathbf{D}}{\partial t}+\mathbf{J} .
\end{aligned}
$$

In linear media the relevant fields satisfy the constitutive relations

$$
\begin{aligned}
& \mathbf{D}=\varepsilon \mathbf{E}, \\
& \mathbf{B}=\mu \mathbf{H} .
\end{aligned}
$$

By taking the divergence of the Ampére law with the Maxwell correction (the fourth equation) and utilizing the Gauss law (the first equation), we find that the current $\mathbf{J}$ must satisfy the continuity equation, namely,

$$
\nabla \cdot \mathbf{J}=0
$$

We then take the curl of the Ampere law with the Maxwell correction and apply a vector identity to obtain

$$
\nabla(\nabla \cdot \mathbf{H})-\Delta \mathbf{H}=\frac{\partial(\nabla \times \mathbf{D})}{\partial t}+\nabla \times \mathbf{J} .
$$

In an isotropic and homogeneous medium (where $\varepsilon$ and $\mu$ are constant scalars), the above equation becomes

$$
\frac{1}{\mu} \nabla(\nabla \cdot \mathbf{B})-\frac{1}{\mu} \Delta \mathbf{B}=\varepsilon \frac{\partial(\nabla \times \mathbf{E})}{\partial t}+\nabla \times \mathbf{J} .
$$

Utilizing the Faraday law in combination with the fact that $\mathbf{B}$ is divergence-free, we obtain

$$
-\frac{1}{\mu} \Delta \mathbf{B}=-\varepsilon \frac{\partial^{2} \mathbf{B}}{\partial t^{2}}+\nabla \times \mathbf{J} .
$$

Finally, we assume that all fields have harmonic timedependence of the form $\mathrm{e}^{\mathrm{i} \omega t}$; in particular, we assume that $\mathbf{B}=\widetilde{\mathbf{B}}(\mathbf{x}) \mathrm{e}^{\mathrm{i} \omega t}$ and $\mathbf{J}=\widetilde{\mathbf{J}}(\mathbf{x}) \mathrm{e}^{\mathrm{i} \omega t}$. We also define

$$
k_{0}=\frac{\omega}{c}=\omega \sqrt{\varepsilon_{0} \mu_{0}} .
$$

Then, thanks to (A.93), we find that $\widetilde{\mathbf{B}}$ satisfies

$$
\Delta \widetilde{\mathbf{B}}+k_{0}^{2} \varepsilon_{r} \mu_{r} \widetilde{\mathbf{B}}=-\mu_{0} \mu_{r} \nabla \times \widetilde{\mathbf{J}},
$$

where $\varepsilon_{r}=\varepsilon / \varepsilon_{0}$ and $\mu_{r}=\mu / \mu_{0}$. Thus each component of $\widetilde{\mathbf{B}}$ satisfies a 3D Helmholtz equation.

A.5.1. 2D Helmholtz Equation. We now assume that the current source $\widetilde{\mathbf{J}}$ is a line current of the form

$$
\widetilde{\mathbf{J}}=\widetilde{J}_{x}(x, y) \mathbf{e}_{x}+\widetilde{J}_{y}(x, y) \mathbf{e}_{y},
$$

where, because $\widetilde{\mathbf{J}}$ must satisfy (A.90),

$$
\frac{\partial \widetilde{J}_{x}}{\partial x}+\frac{\partial \widetilde{J}_{y}}{\partial y}=0 .
$$

By symmetry, none of the fields will depend on $z$. In addition, we assume that we are dealing with nonmagnetic materials for which $\mu_{r}=1$. Thus (A.95) and (A.96) imply that the $z$ component of $\widetilde{\mathbf{B}}$ satisfies

$$
\frac{\partial^{2} \widetilde{B}_{z}}{\partial x^{2}}+\frac{\partial^{2} \widetilde{B}_{z}}{\partial y^{2}}+k_{0}^{2} \varepsilon_{r} \widetilde{B}_{z}=-\mu_{0}\left(\frac{\partial \widetilde{J}_{y}}{\partial x}-\frac{\partial \widetilde{J}_{x}}{\partial y}\right) .
$$


Finally, the Maxwell equations can be used to show that $\widetilde{B}_{z}$ and $\varepsilon_{r}^{-1}\left(\partial \widetilde{B}_{z} / \partial x\right)$ must be continuous across the boundaries of the slab at $x=0$ and $x=a$ [26]. Then (A.98) can be written in divergence form as

$$
\nabla \cdot\left(\frac{1}{\varepsilon_{r}} \nabla \widetilde{B}_{z}\right)+k_{0}^{2} \widetilde{B}_{z}=-f
$$

where

$$
f \equiv \mu_{0}\left(\frac{\partial \tilde{J}_{y}}{\partial x}-\frac{\partial \widetilde{J}_{x}}{\partial y}\right)
$$

\section{Competing Interests}

The authors declare that there are no conflicts of interests regarding the publication of this article.

\section{Acknowledgments}

The research of Andrew E. Thaler was supported in part by the Institute for Mathematics and Its Applications with funds provided by the National Science Foundation through NSF Award 0931945 and in part by AFOSR under the 2013 YIP Award FA9550-13-1-0078.

\section{References}

[1] G. W. Milton, N.-A. P. Nicorovici, R. C. McPhedran, and V. A. Podolskiy, "A proof of superlensing in the quasistatic regime, and limitations of superlenses in this regime due to anomalous localized resonance," Proceedings of the Royal Society of A: Mathematical, Physical and Engineering Sciences, vol. 461, no. 2064, pp. 3999-4034, 2005.

[2] N. A. Nicorovici, R. C. McPhedran, and G. W. Milton, "Optical and dielectric properties of partially resonant composites," Physical Review B, vol. 49, no. 12, pp. 8479-8482, 1994.

[3] M. Yan, W. Yan, and M. Qiu, "Cylindrical superlens by a coordinate transformation," Physical Review B-Condensed Matter and Materials Physics, vol. 78, no. 12, Article ID 125113, 2008.

[4] D. J. Bergman, "Perfect imaging of a point charge in the quasistatic regime," Physical Review A, vol. 89, no. 1, Article ID 015801, 2014.

[5] H.-M. Nguyen, "Superlensing using complementary media," Annales de l'Institut Henri Poincaré (C) Non Linear Analysis, vol. 32, no. 2, pp. 471-484, 2015.

[6] J. B. Pendry, "Negative refraction makes a perfect lens," Physical Review Letters, vol. 85, no. 18, pp. 3966-3969, 2000.

[7] J. B. Pendry and S. A. Ramakrishna, "Near-field lenses in two dimensions," Journal of Physics: Condensed Matter, vol. 14, no. 36, pp. 8463-8479, 2002.

[8] G. W. Milton and N. P. Nicorovici, "On the cloaking effects associated with anomalous localized resonance," Proceedings of the Royal Society of A: Mathematical, Physical and Engineering Sciences, vol. 462, no. 2074, pp. 3027-3059, 2006.

[9] H. Ammari, G. Ciraolo, H. Kang, H. Lee, and G. W. Milton, "Anomalous localized resonance using a folded geometry in three dimensions," Proceedings of the Royal Society of A: Mathematical, Physical and Engineering Sciences, vol. 469, no. 2154, Article ID 20130048, 2013.

[10] R. V. Kohn, J. Lu, B. Schweizer, and M. I. Weinstein, "A variational perspective on cloaking by anomalous localized resonance," Communications in Mathematical Physics, vol. 328, no. 1, pp. 1-27, 2014.

[11] H. Ammari, G. Ciraolo, H. Kang, H. Lee, and G. W. Milton, "Spectral theory of a Neumann-Poincaré-type operator and analysis of cloaking due to anomalous localized resonance," Archive for Rational Mechanics and Analysis, vol. 208, no. 2, pp. 667-692, 2013.

[12] H. Ammari, G. Ciraolo, H. Kang, H. Lee, and G. W. Milton, "Spectral theory of a Neumann-Poincaré-type operator and analysis of cloaking due to anomalous localized resonance," Archive for Rational Mechanics and Analysis, vol. 208, no. 2, pp. 667-692, 2013.

[13] G. Bouchitté and B. Schweizer, "Cloaking of small objects by anomalous localized resonance," The Quarterly Journal of Mechanics and Applied Mathematics, vol. 63, no. 4, pp. 437-463, 2010.

[14] O. P. Bruno and S. Lintner, "Superlens-cloaking of small dielectric bodies in the quasistatic regime," Journal of Applied Physics, vol. 102, no. 12, Article ID 124502, 2007.

[15] R. C. McPhedran, N.-A. P. Nicorovici, L. C. Botten, and G. W. Milton, "Cloaking by plasmonic resonance among systems of particles: cooperation or combat?" Comptes Rendus Physique, vol. 10, no. 5, pp. 391-399, 2009.

[16] M. Xiao, X. Huang, J.-W. Dong, and C. T. Chan, "On the time evolution of the cloaking effect of a metamaterial slab," Optics Letters, vol. 37, no. 22, pp. 4594-4596, 2012.

[17] H.-M. Nguyen, "Cloaking via anomalous localized resonance. A connection between the localized resonance and the blow up of the power for doubly complementary media," Comptes Rendus Mathematique, vol. 353, no. 1, pp. 41-46, 2015.

[18] G. W. Milton, N.-A. P. Nicorovici, and R. C. McPhedran, “Opaque perfect lenses," Physica B: Condensed Matter, vol. 394, no. 2, pp. 171-175, 2007.

[19] A. D. Yaghjian and T. B. Hansen, "Plane-wave solutions to frequency-domain and time-domain scattering from magnetodielectric slabs," Physical Review E, vol. 73, Article ID 046608, 2006.

[20] N.-A. P. Nicorovici, R. C. McPhedran, S. Enoch, and G. Tayeb, "Finite wavelength cloaking by plasmonic resonance," New Journal of Physics, vol. 10, Article ID 115020, 2008.

[21] H. Kettunen, M. Lassas, and P. Ola, "On absence and existence of the anomalous localized resonance without the quasi-static approximation," http://arxiv.org/abs/1406.6224.

[22] H.-M. Nguyen, "Cloaking via anomalous localized resonance for doubly complementary media in the quasistatic regime," Journal of the European Mathematical Society, vol. 17, no. 6, pp. 1327-1365, 2015.

[23] A. E. Thaler, Bounds on the volume of an inclusion in a body and cloaking due to anomalous localized resonance [Ph.D. thesis], University of Utah, Salt Lake City, Utah, USA, 2014.

[24] T. Meklachi, G. W. Milton, D. Onofrei, A. E. Thaler, and G. Funchess, "Sensitivity of anomalous localized resonance phenomena with respect to dissipation," Quarterly of Applied Mathematics, vol. 74, no. 2, pp. 201-234, 2016. 
[25] G. N. Watson, A Treatise on the Theory of Bessel Functions, Cambridge University Press, Cambridge, UK, 1996.

[26] D. J. Griffths, Introduction to Electrodynamics, Prentice-Hall, Upper Saddle River, NJ, USA, 3rd edition, 1999. 


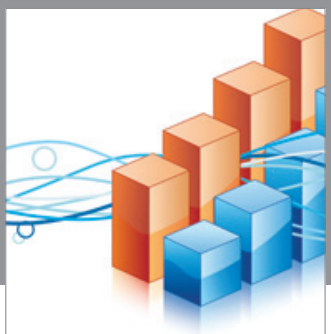

Advances in

Operations Research

vatem alat4

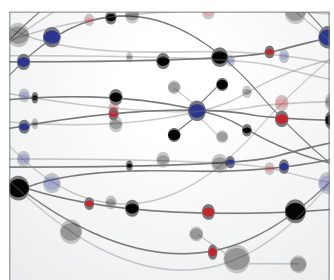

\section{The Scientific} World Journal
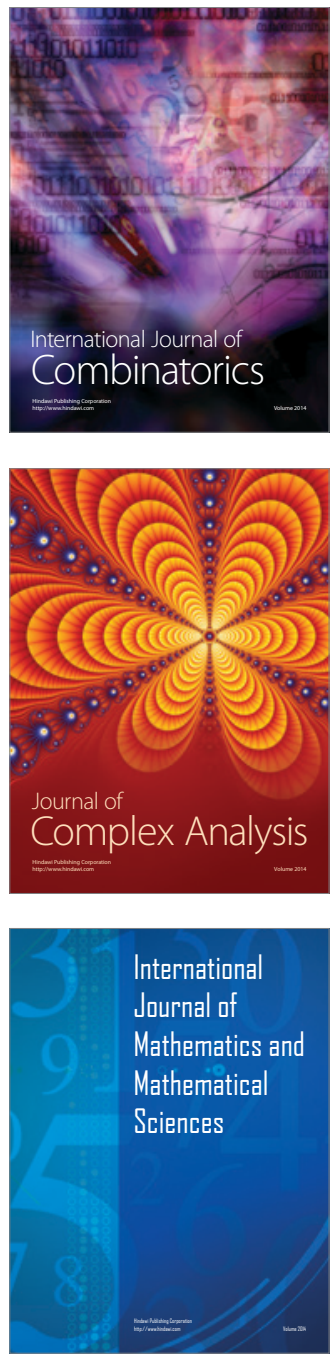
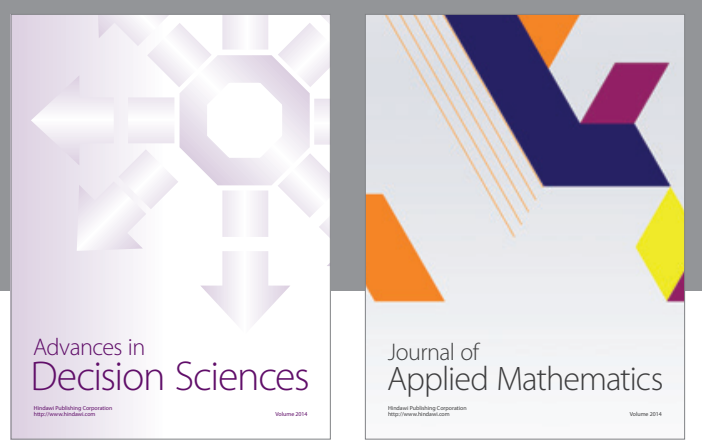

Algebra

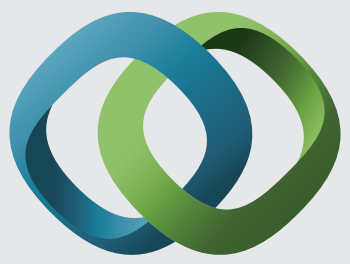

\section{Hindawi}

Submit your manuscripts at

http://www.hindawi.com
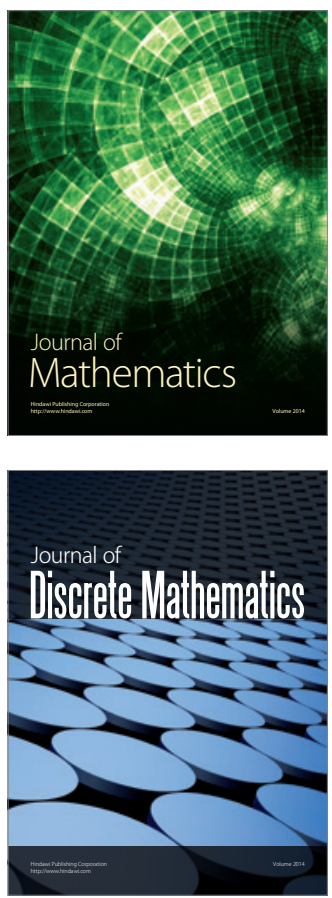

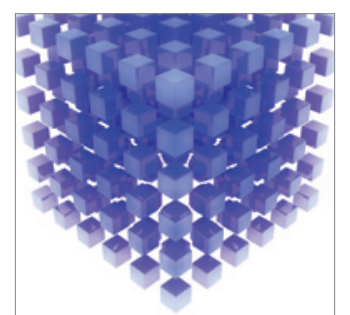

Mathematical Problems in Engineering
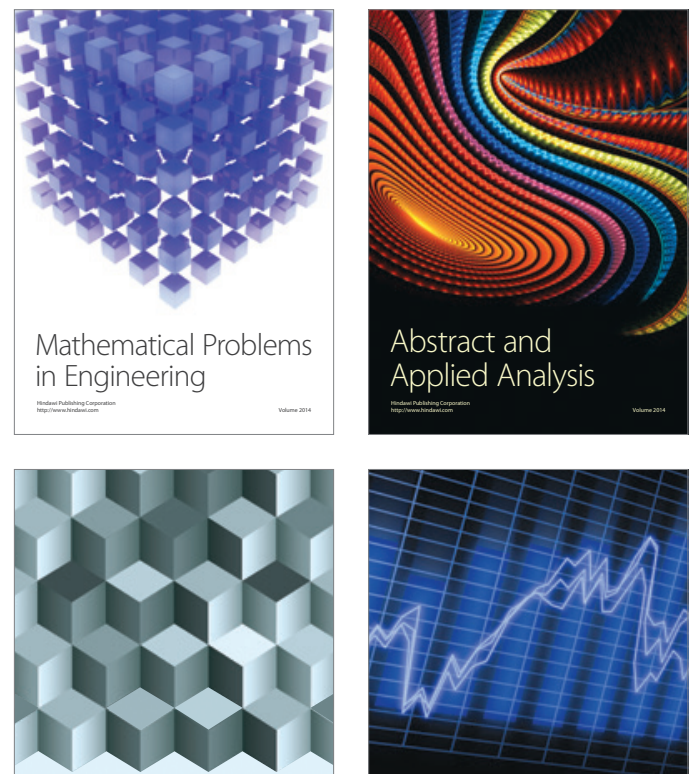

Journal of

Function Spaces

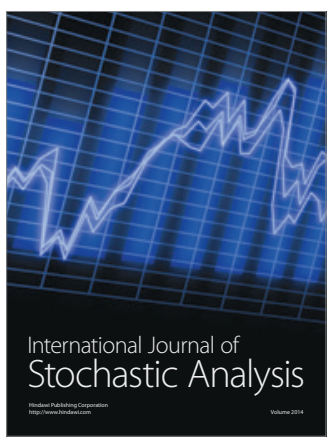

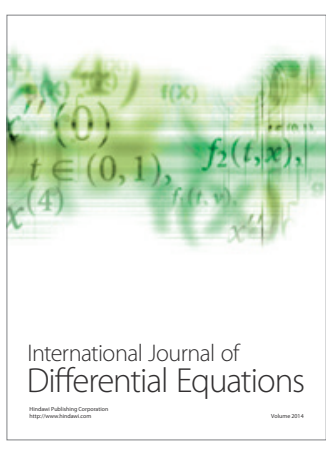
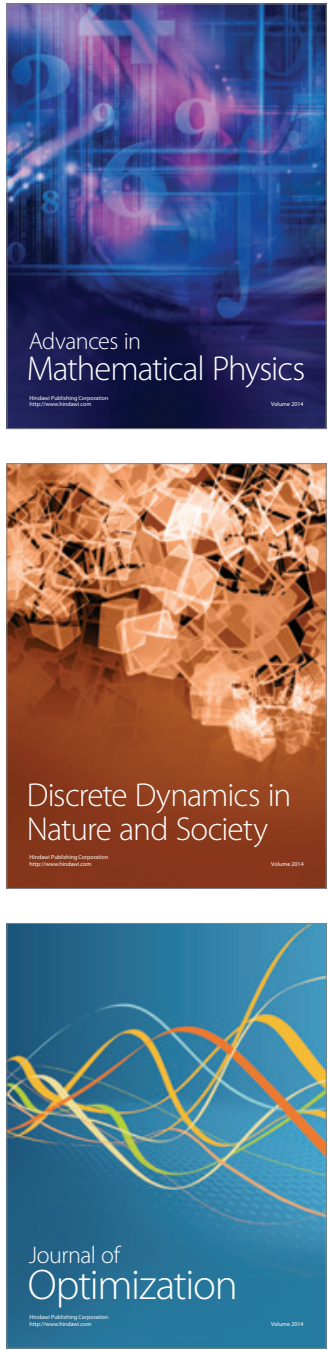